(c) 2000 International Press

Adv. Theor. Math. Phys. 1 (2000) 1-94

\title{
Quantum Geometry of
}

\section{Isolated Horizons and Black}

\section{Hole Entropy}

Abhay Ashtekar ${ }^{1,2}$, John C. Baez ${ }^{3,1}$, and Kirill Krasnov ${ }^{2,4}$

${ }^{1}$ Center for Gravitational Physics and Geometry Department of Physics, The Pennsylvania State University

University Park, PA 16802, USA

${ }^{2}$ Institute of Theoretical Physics

University of California, Santa Barbara, CA 93106, USA

${ }^{3}$ Department of Mathematics, University of California

Riverside, CA 92521, USA

${ }^{4}$ Department of Physics, University of California

Santa Barbara, CA 93106, USA

ashtekar@gravity.phys.psu.edu

baez@math.ucr.edu

krasnov@cosmic.physics.ucsb.edu

e-print archive: http://xxx.lanl.gov/gr-qc/0005126 


\begin{abstract}
Using the classical Hamiltonian framework of [1] as the point of departure, we carry out a non-perturbative quantization of the sector of general relativity, coupled to matter, admitting non-rotating isolated horizons as inner boundaries. The emphasis is on the quantum geometry of the horizon. Polymer excitations of the bulk quantum geometry pierce the horizon endowing it with area. The intrinsic geometry of the horizon is then described by the quantum Chern-Simons theory of a U(1) connection on a punctured 2-sphere, the horizon. Subtle mathematical features of the quantum Chern-Simons theory turn out to be important for the existence of a coherent quantum theory of the horizon geometry. Heuristically, the intrinsic geometry is flat everywhere except at the punctures. The distributional curvature of the $\mathrm{U}(1)$ connection at the punctures gives rise to quantized deficit angles which account for the overall curvature. For macroscopic black holes, the logarithm of the number of these horizon microstates is proportional to the area, irrespective of the values of (non-gravitational) charges. Thus, the black hole entropy can be accounted for entirely by the quantum states of the horizon geometry. Our analysis is applicable to all nonrotating black holes, including the astrophysically interesting ones which are very far from extremality. Furthermore, cosmological horizons (to which statistical mechanical considerations are known to apply) are naturally incorporated.

An effort has been made to make the paper self-contained by including short reviews of the background material.
\end{abstract}

\title{
1 Introduction
}

Isolated horizons are a generalization of the event horizons of stationary black holes to physically more realistic situations $[1,2]$. The generalization is in two directions. First, while one needs the entire spacetime history to locate an event horizon, isolated horizons are defined using local spacetime structures. Second, spacetimes with isolated horizons need not admit any Killing field. Thus, although the horizon itself is stationary, the outside spacetime can contain non-stationary fields and admit radiation. This feature mirrors the physical expectation that, as 
in statistical mechanics of ordinary systems, a discussion of the equilibrium properties of black holes should only require the black hole to be in equilibrium and not the whole universe. These generalizations are also mathematically significant. For example, while the space of stationary solutions to the Einstein-Maxwell equations admitting event horizons is three dimensional, the space of solutions admitting isolated horizons is infinite dimensional. Yet, the structure available on isolated horizons is sufficiently rich to allow a natural extension of the standard laws of black hole mechanics [2, 3]. Finally, cosmological horizons to which thermodynamic considerations also apply [4] are special cases of isolated horizons.

It is then natural to ask if one can analyze the quantum geometry of isolated horizons in detail and account for entropy from statistical mechanical considerations. We will see that the answer to both questions is in the affirmative. (A summary of these results appeared in [5]. For early work, see $[6,7]$.)

The first paper in this series [1] introduced the notion of an undistorted, non-rotating, isolated horizon and examined 4-dimensional spacetimes which are asymptotically flat and admit such an isolated horizon as inner boundary. If one chooses a partial Cauchy surface $M$ in such a spacetime, the intersection of $M$ with the horizon will be a 2 -sphere $S$, as shown in Figure 1 . In $[1,2]$, the Hamiltonian framework adapted to such 3 -manifolds with internal boundaries was constructed starting from a suitable action principle. This framework has the novel feature that the expression for the gravitational symplectic structure contains, in addition to the familiar volume integral over $M$, a surface term given as an integral over the boundary $S$. Furthermore, this surface term coincides with the standard symplectic structure of the $\mathrm{U}(1)$ Chern-Simons theory, where the $\mathrm{U}(1)$ connection $W$ is simply the gravitational spin-connection on the horizon 2 -sphere. ${ }^{1}$ This framework offers a natural point of departure for non-perturbative quantization.

The purpose of this paper is to carry out this quantization in detail, examine the resulting quantum geometry of isolated horizons and

\footnotetext{
${ }^{1}$ In [1] this $\mathrm{U}(1)$ connection was called $V$ instead of $W$. In this paper $V$ is generally used as a suffix to label structures associated with volume fields defined on $M$ and $S$ as a suffix which labels structures associated with surface fields defined on $S$.
} 


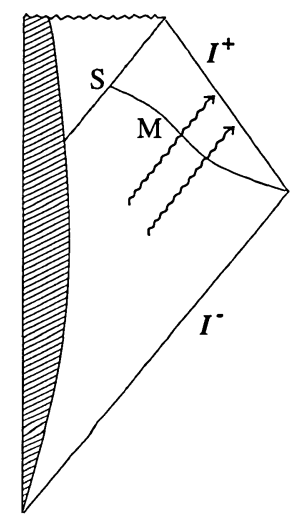

Figure 1: A typical spacetime of interest depicting a gravitational collapse. The horizon geometry becomes time-independent at late times, say to the future of a cross-section $S$. However, there is gravitational radiation crossing any (partial) Cauchy surface $M$ in the exterior region.

use the associated quantum states to calculate the statistical mechanical entropy of these horizons in the context of non-perturbative quantum gravity. Conceptually, perhaps the most striking feature of this description is that ideas from three distinct sources - the analysis of isolated horizons in classical general relativity [1], the quantum theory of geometry [8-24], and the U(1) Chern-Simons theory - fuse together seamlessly to provide a coherent description of the quantum states of isolated horizons. At certain points there is even a delicate matching between numerical coefficients calculated independently within these areas, suggesting that the underlying unity is potentially deep; its ramifications are yet to be fully understood. From a practical viewpoint, the most notable feature is that the framework can incorporate, in a single stroke, a wide variety of horizons, without any restriction on near-extremality, on the ratio of the horizon radius to the cosmological radius, etc. made in other approaches.

We have attempted to make this paper self-contained. In particular, for the convenience of readers who may not be interested in the intricacies of global issues in classical general relativity discussed in $[1,2]$, in Section 2 we review the background material needed here. This section is divided into two parts. In the first, we recall from [1] the Hamiltonian framework which serves as the point of departure for quantization. As 


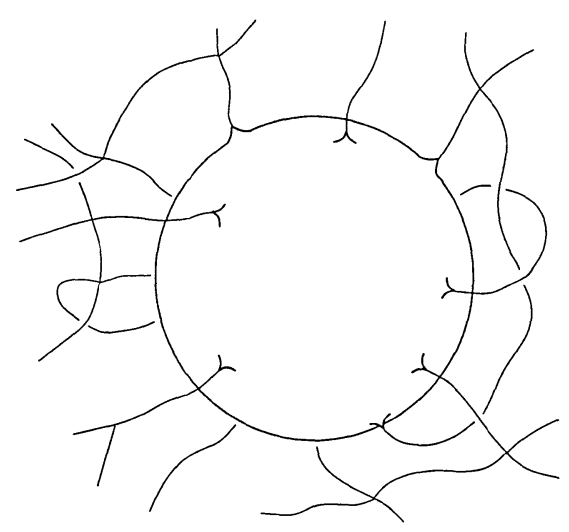

Figure 2: Quantum Horizon. Polymer excitations in the bulk puncture the horizon, endowing it with quantized area. Intrinsically, the horizon is flat except at punctures where it acquires a quantized deficit angle. These angles add up to $4 \pi$.

usual, the phase space consists of certain smooth fields on a 3-manifold $M .{ }^{2}$ The horizon boundary conditions imply that the only independent degree of freedom on $S$ is a $\mathrm{U}(1)$ connection $W$. Note however that in the classical theory $W$ does not represent a new degree of freedom; it is determined by the limiting value of the connection in the bulk. But, as mentioned above, the symplectic structure is somewhat unusual: in addition to the familiar volume term, it contains also a Chern-Simons term for $W$ on the internal boundary $S$. In the second part of this section, we recall the quantum theory of geometry [8-24] which has been developed on manifolds without boundary. In this theory, the fundamental excitations are the Wilson lines of an $\mathrm{SU}(2)$ connection $A$. Physically, these Wilson lines can be thought of as 'flux lines of area': heuristically, they endow each surface which they intersect with a quantum of area. Because of the one-dimensional nature of these excitations, the resulting quantum geometry is often referred to as 'polymer geometry'.

The first goal of this paper is to extend this quantum geometry to situations in which there is an inner boundary $S$ representing the

\footnotetext{
${ }^{2}$ As in $[1,2]$, here we will be interested primarily in non-rotating isolated horizons. The classical theory needed in the treatment of more general cases is now well-understood [3] and the quantum theory will be discussed in subsequent papers. For brevity, in the rest of this paper, $S$ will be referred to simply as the 'horizon'.
} 


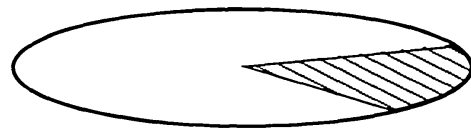

(a)

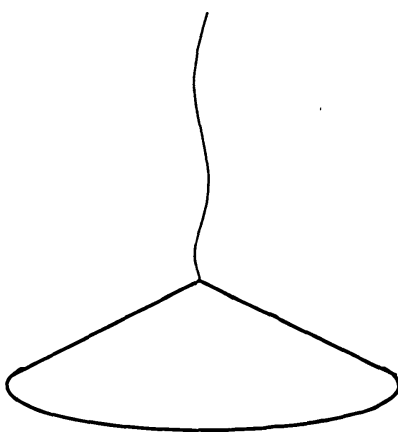

(b)

Figure 3: (a) Deficit angle in the intrinsic horizon 2-geometry. (b) A 3-dimensional perspective: a bulk polymer excitation 'exerts a tug' on the horizon causing a deficit angle.

horizon. The overall strategy is summarized in section 3. Section 4 discusses the kinematical Hilbert space. Recall that in quantum field theories with local degrees of freedom, quantum states are functions of generalized fields which need not be continuous. Therefore, we are led to consider generalized connections $[11,12]$ whose behavior on the boundary $S$ can be quite independent of their behavior in the bulk. Thus, there is an interesting and important departure from the situation in the classical theory. As a result, surface states are no longer determined by the volume states. Rather, the total Hilbert space is a subspace of the tensor product $\mathcal{H}_{V} \otimes \mathcal{H}_{S}$ of a volume Hilbert space $\mathcal{H}_{V}$ with a surface Hilbert space $\mathcal{H}_{S}$. States in the volume Hilbert space $\mathcal{H}_{V}$ represent polymer geometries as before. However, now the one-dimensional excitations can end on $S$ where they make punctures. (See Figure 2.) At each puncture, they induce a specific distributional curvature for the $\mathrm{U}(1)$ connection $W$. Furthermore, the space of $\mathrm{U}(1)$ connections on $S$ naturally inherits the Chern-Simons symplectic structure from the classical Hamiltonian framework. Therefore, the two pieces of information needed in the quantization of Chern-Simons theory on a punctured surface are now at hand, one supplied by the quantum theory of geometry, and the other by the classical theory of isolated horizons; the three theories are naturally intertwined. The surface Hilbert space $\mathcal{H}_{S}$ is the space of states of this Chern-Simons theory.

Section 5 extracts physical states by imposing the quantum version 
of the phase space boundary conditions and constraints on the space of kinematical states. It turns out that the quantum boundary conditions ensure that the volume and the surface states are coupled just in the correct way to ensure gauge invariance of the total state. This is another illustration of the unexpected and delicate matching between the isolated horizon boundary conditions from classical general relativity, quantum geometry and the quantum Chern-Simons theory. The quantum geometry of the horizon emerges from this interplay. The holonomy of the $\mathrm{U}(1)$ connection $W$ around a loop in $S$ is non-trivial only if the loop encloses a puncture. The holonomy around each puncture endows it with a deficit angle, as depicted in Figure 3. All these angles are quantized and add up, in a suitable sense, to $4 \pi$. Thus, heuristically, one can say that the quantum horizon is flat except at the punctures.

The second goal of the paper is to use this quantum horizon geometry to account for the entropy of isolated horizons. This task is carried out in Section 6. Note that the physical states considered so far include information about gravitational and electromagnetic radiation far away from the black hole which is obviously irrelevant to the calculation of black hole entropy. What is relevant are the states directly associated with the horizon of a given area, say $a_{0}$. One is therefore led to trace over the volume degrees of freedom and construct a density matrix $\rho_{\mathrm{bh}}$ describing a maximum entropy mixture of surface states for which the area of the horizon $a$ lies in the range $a_{0}-\delta \leq a \leq a_{0}+\delta$ for some small $\delta\left(\sim \ell_{P}^{2}\right)$, and for which the electric, magnetic and dilaton charges lie in similar small intervals containing the classical value. The statistical mechanical entropy is then given by $S_{\mathrm{bh}}=-\operatorname{Tr}\left(\rho_{\mathrm{bh}} \ln \rho_{\mathrm{bh}}\right)$. As usual, this number can be calculated simply by counting states: $S_{\text {bh }}=\ln N_{\text {bh }}$ where $N_{\text {bh }}$ is the number of Chern-Simons surface states satisfying the area and charge constraints. We find that, irrespective to the values of charges, for $a_{0} \gg \ell_{P}^{2}$, the (leading term in the expression of the) entropy is proportional to the area. Thus, the entropy can be traced back directly to the quantum states of the geometry of the horizon, states which can interact with the other degrees of freedom in the physical, curved spacetime geometry around the black hole. There are no corresponding surface states for Maxwell or dilaton fields. Section 7 summarizes the results and compares and contrasts our approach to other approaches available in the literature. 
As discussed in detail in [1,2], isolated horizons encompass a wider class of situations than black holes. In particular, they also include 'cosmological horizons' of the type encountered in the deSitter spacetime which has no black hole at all. It has been known for some time [4] that thermodynamic considerations apply also to cosmological horizons and recent work $[2,3]$ has shown that the laws of black hole mechanics extends to all isolated horizons. Therefore, one would expect a statistical mechanical description to extend to this wider class of horizons. Results of this paper will show that this expectation is correct. Furthermore, there exists a single statistical mechanical framework that unifies these apparently distinct situations. However, for simplicity of presentation, we will focus on black holes in the main body of the paper and briefly discuss the more general cases in Section 7 .

For convenience of the reader, we have organized the material such that the background material and the main results are described in Sections 2, 3 and 6.1 and 6.3. These sections can be read independently of others which contain proofs and technical subtleties. An index of notation is included at the end of the paper.

\section{Preliminaries}

This section is divided into two parts. In the first, we briefly review the classical Hamiltonian framework of [1] for general relativity in presence of an inner boundary representing an isolated horizon. In the second, we sketch the quantum theory of Riemannian geometry on manifolds without boundary developed in [9-24]. In sections 3,4 and 5, these two ingredients will be combined and further developed to obtain the quantum geometry of isolated horizons.

\subsection{Classical Hamiltonian framework}

The non-perturbative quantization used in this paper is based on a Hamiltonian framework. Therefore, the starting point is the classical phase space. The arena is a 3-manifold $M$, the complement of the unit open ball in $\mathbb{R}^{3}$. The boundary of $M$ is a 2 -sphere, which we denote by $S$. We think of $M$ as the partial Cauchy surface exterior to the black 
hole, and think of $S$ as the intersection of an isolated horizon with $M$ (see Figure 1 for a prototype situation). For simplicity we will refer to $S$ simply as the 'horizon' and use under-bars to denote the pullback of fields from $M$ to $S$.

Let $P$ be the trivial SU(2) bundle over $M$. The fields of our classical system are a connection $A$ on $P$ and an $A d P$-valued 2 -form $\Sigma$ on $M$. We denote the curvature of $A$ by $F$. We may identify $A$ with an $\mathfrak{s u}(2)$ valued 1-form and identify $\Sigma$ and $F$ with $\mathfrak{s u}(2)$-valued 2-forms. We use lower-case Roman letters $a, b, c, \ldots$ for spatial tensor indices and lowercase Roman letters $i, j, k, \ldots$ for 'internal' indices running over a basis of $\mathfrak{s u}(2)$. Thus in component notation we write $A$ as $A_{a}^{i}$ and $\Sigma$ as $\Sigma_{a b}^{i}$. The internal indices are raised and lowered using the Cartan-Killing form on $\mathfrak{s u}(2)$. However, we often suppress the internal indices ${ }^{3}$. The connections may be thought of as the configuration variables and $\Sigma$ as their canonical momenta. Thus the kinematical phase space is the same as in SU(2) Yang-Mills theory, the duals of the 2-forms $\Sigma$ playing the role of the Yang-Mills electric fields. However, the interpretation of these fields is quite different. Roughly, the connections can be thought of as 'gravitational spin connections' and their momenta as 'spatial triads'. However, there are a number of technical subtleties [25, 26]. The final picture can be summarized as follows.

For each positive number $\gamma$, there exists a phase space ${ }^{\gamma \mathcal{X}}$ consisting of pairs $\left({ }^{\gamma} A,{ }^{\gamma} \Sigma\right)$ of smooth fields on $M$ satisfying certain boundary conditions. $\gamma$ is called the Barbero-Immirzi parameter and in some ways is analogous to the $\theta$-parameter in Yang-Mills theories. These phase spaces are naturally isomorphic to one another. One can write Einstein's equations using any value of $\gamma$. They have the same general form and the same physical content at the classical level; the difference lies only in certain relative numerical factors involving $\gamma$ between various terms. Therefore, at the classical level, without loss of generality one can just work with a fixed value of $\gamma$ and, if so desired, pass to another $\gamma$-sector via a canonical transformation. However, in

\footnotetext{
${ }^{3}$ In the previous paper [1] which dealt with the classical theory, it was more convenient to work in the spin- $\frac{1}{2}$ representation of $\mathrm{SU}(2)$. There, the basic fields were denoted by $A_{a A}{ }^{B}$ and $\Sigma_{a b A}{ }^{B}$ in the component notation. The relation between these fields and those in this paper is given by: $A_{a A}{ }^{B}=-\frac{i}{2} A_{a}^{i} \tau_{i A}{ }^{B}$ and $\Sigma_{a b A}{ }^{B}=$ $-\frac{i}{2} \Sigma_{a b}^{i} \tau_{i A}{ }^{B}$, where $\tau_{i A}{ }^{B}$ are the $2 \times 2$ traceless, Hermitian matrices satisfying $\tau_{i} \tau_{j}=$ $i \epsilon_{i j k} \tau_{k}+\delta_{i j} 1$.
} 
the quantum theory, these canonical transformations fail to be unitarily implementable. Hence distinct $\gamma \mathcal{X}$ lead to unitarily inequivalent quantum theories. These theories are physically distinct because, as we explain in Section 2.2, the spectra of geometric operators, such as those defining surface-areas, depend on $\gamma$ and are distinct in different $\gamma$-sectors. As with the $\theta$ parameter in quantum chromodynamics, the value of $\gamma$ can be determined only experimentally, or via new theoretical inputs. Therefore, for our purposes it is important to explore all $\gamma$ sectors.

The geometrical meaning of the fields $\left({ }^{\gamma} A,{ }^{\gamma} \Sigma\right)$ is as follows. Starting from the 2 -forms ${ }^{\gamma} \Sigma$ one can define an orthonormal triad $E$ of density weight one on $M$ as follows:

$$
E_{i}^{a}:=\gamma \eta^{a b c}\left({ }^{\gamma} \Sigma_{b c i}\right)
$$

where $\eta^{a b c}$ is the metric-independent Levi-Civita 3-form of density weight one. Thus, the 3-metric $q_{a b}$ on $M$ is given by

$$
E_{i}^{a} E^{b i}=\mathbf{q} q^{a b}
$$

where $\mathbf{q}$ is the determinant of the metric $q_{a b}$. The triad determines an unique torsion-free derivative operator $D$ which acts on both, tensor and internal indices. Denote by $\Gamma_{a}^{i}$ the corresponding $\mathrm{SU}(2)$ connection on $P$ and by $K_{a}^{i}$ the $\operatorname{Ad} P$-valued 1-form constructed from the extrinsic curvature $K_{a b}$ of $M$ via $K_{a}^{i}=(1 / \sqrt{\mathbf{q}}) K_{a b} E^{b i}$. In the spacetime picture, the connection $\Gamma$ is constructed from spatial derivatives of the triad $E$ while the 1-forms $K$ determine their time derivatives. In terms of these fields, our phase space variable ${ }^{\gamma} A$ is given by:

$$
{ }^{\gamma} A_{a}:=\Gamma_{a}-\gamma K_{a}
$$

it depends on spatial and temporal derivatives of the triads. For notational simplicity, from now on we will drop the suffix $\gamma$. Thus, unless otherwise stated, from now on $(A, \Sigma)$ will uniformly denote $\left({ }^{\gamma} A,{ }^{\gamma} \Sigma\right)$.

The boundary conditions at infinity ensure that the geometry is asymptotically flat at spatial infinity $[1,2]$. Since they will not play an essential role in the present paper, we will not state them explicitly. The structure at the horizon, on the other hand, will play a crucial role. To spell it out, let us first note that the geometrical interpretation (1) 
of $\Sigma$ provides a formula for area of any 2 -surface $T$ in $M$.

$$
A_{T}=\gamma \int_{T}\left(\tilde{\Sigma}^{i} \tilde{\Sigma}^{j} k_{i j}\right)^{\frac{1}{2}} d^{2} x
$$

where $\tilde{\Sigma}^{i}=\eta^{a b} \Sigma_{a b}^{i}$ with $\eta^{a b}$ the (metric-independent) Levi-Civita density on $T$, and where, as before $k_{i j}$ is the Cartan-Killing metric on $\mathfrak{s u}(2)$. The integral is well-defined because the integrand is a density of weight one; it gives the area because the integrand equals the square root of the determinant of the 2-metric induced on $T$.

We can now state the horizon boundary conditions. First, only those 2 -forms $\Sigma$ are admissible for which the horizon area has a fixed value, $a_{0}$. Second, the pullback $\underline{A}$ of $A$ to $S$ is completely determined by a U(1) connection $W$ on $S$ and the constant $a_{0}$. The third and final boundary condition is that the pullback $\underline{\Sigma}$ of $\Sigma$ to $S$ is completely determined by the curvature $F=d W$ of $W$.

To specify these restrictions explicitly, let us first fix a smooth function $r: S \rightarrow \mathfrak{s u}(2)$ with $|r|=1$ which has degree 1 when viewed as a map from a 2-sphere to itself. Clearly, $r$ is fixed by a U(1) subgroup of the gauge group at each point of $S$. It thus picks out a U(1) sub-bundle $Q$ of the $\mathrm{SU}(2)$ bundle $\left.P\right|_{S}$ given by restricting $P$ to $S$. (Intrinsically, $Q$ is just the spin bundle of $S$.) Then $W$ is a connection on $Q$, defined in terms of the $\mathrm{SU}(2)$ spin connection $\Gamma$ on $P$ via

$$
W_{a}:=-\frac{1}{\sqrt{2}} \underline{\Gamma}_{a}^{i} r_{i}
$$

and its curvature $F$ is related to $\Sigma$ via

$$
F_{a b}=-\frac{2 \pi \gamma}{a_{0}} \Sigma_{a b}^{i} r_{i}
$$

The phase space $\mathcal{X}$ consists of pairs $(A, \Sigma)$ of asymptotically flat, smooth fields on $M$ satisfying the horizon boundary conditions just stated. $\mathcal{X}$ is an infinite-dimensional smooth manifold - technically, a Frechét submanifold of the vector space of smooth $(A, \Sigma)$ pairs equipped with a suitable topology defined using the Cartesian components of $A$ and $\Sigma$. The symplectic structure on $\mathcal{X}$ is given by

$$
\begin{aligned}
& \Omega_{\text {grav }}\left((\delta A, \delta E),\left(\delta A^{\prime}, \delta E^{\prime}\right)\right) \\
& =\frac{1}{8 \pi G}\left[\int_{M} \operatorname{Tr}\left(\delta A \wedge \delta^{\prime} \Sigma-\delta^{\prime} A \wedge \delta \Sigma\right)+\frac{a_{0}}{\gamma \pi} \oint_{S} \delta W \wedge \delta^{\prime} W\right]
\end{aligned}
$$


for any tangent vectors $(\delta A, \delta \Sigma)$ and $\left(\delta A^{\prime}, \delta E^{\prime}\right)$ at a point of $\mathcal{X}$. Note that, in addition to the familiar volume term, this symplectic structure has a surface term which coincides with the symplectic structure of the $\mathrm{U}(1)$ Chern-Simons theory. The symplectic structure $\Omega_{\text {grav }}$ is weakly nondegenerate - that is, a tangent vector to $\mathcal{X}$ whose pairing with any other tangent vector vanishes must be zero.

The phase space $\mathcal{X}$ serves as the arena for the Hamiltonian formulation of general relativity. Points of $\mathcal{X}$ represent 'kinematical states'; they are not all physically realized. This is because some of Einstein's equations contain no time derivatives and thus constrain the physically admissible states to lie in a submanifold $\tilde{\mathcal{X}}$ of $\mathcal{X}$. The constraint submanifold $\tilde{\mathcal{X}}$ is defined by three sets of restrictions, called the Gauss, diffeomorphism and Hamiltonian constraints. They are of first class in Dirac's terminology: the pullback of the symplectic structure to the $\tilde{\mathcal{X}}$ is degenerate and the degenerate directions correspond precisely to the Hamiltonian vector fields generated by the constraint functions. Therefore, motions generated by these vector fields can be interpreted as gauge transformations in the phase space. A careful analysis [1] shows that they correspond to: i) $\mathrm{SU}(2)$ internal rotations on $(A, \Sigma)$ that reduce to $\mathrm{U}(1)$ rotations preserving $r$ on the boundary $S$; ii) spatial diffeomorphisms generated by vector fields which are tangential to $S$; and, iii) 'bubble-time evolution' in spacetime where the 3 -surfaces $M$ are kept fixed both at the horizon and at infinity. It turns out that the boundary condition (6) plays an important role in ensuring full invariance with respect to the $\mathrm{SU}(2)$ internal rotations on the boundary. Without it, as in the case of the Hamiltonian constraint, only those internal rotations whose generators vanish on $S$ could be regarded as gauge.

Finally, note that we can define a 'volume phase space' $\mathcal{X}_{V}$ and a 'surface phase space' $\mathcal{X}_{S}$ as follows. $\mathcal{X}_{V}$ is isomorphic with $\mathcal{X}$ as a manifold but it is equipped with a symplectic structure $\Omega_{V}$ given just by the volume term in (7). $\mathcal{X}_{S}$ is the space of $\mathrm{U}(1)$ connections $W$ on $S$ equipped with the Chern-Simons symplectic structure - i.e., the surface term in (7). Then, we have natural maps

$$
p_{V}: \mathcal{X} \rightarrow \mathcal{X}_{V}, \quad p_{S}: \mathcal{X} \rightarrow \mathcal{X}_{S}
$$

given by

$$
p_{V}(A, \Sigma)=(A, \Sigma) \quad p_{S}(A, \Sigma)=W
$$


such that

$$
\Omega_{\text {grav }}=p_{V}^{*} \Omega_{V}+p_{S}^{*} \Omega_{S} .
$$

We will see that this structure on the kinematical space of classical states is faithfully mirrored in the kinematical space of quantum states.

\subsection{Quantum geometry}

In general relativity, geometry is treated as a physical entity with degrees of freedom of its own. Indeed, some of the most dramatic predictions of the theory - black holes and gravitational waves - center around purely geometric notions. This viewpoint plays a central role in non-perturbative quantum gravity. Thus, unlike in other approaches such as string theory, one avoids the introduction of a classical, background geometry on which quantum matter is to live. Rather, matter and geometry are both treated quantum mechanically from the very beginning. Quantum gravity is regarded as a theory of quantum geometry interacting with quantum matter. In this section, we will review the relevant features of quantum geometry on a spatial 3-manifold $M$ without boundary. Modifications required by the presence of the internal boundary at the horizon will be discussed in Section 4 .

Recall that in quantum field theory states are often described by square-integrable wave functions, not on the classical configuration space of smooth fields, but on certain completion of this which also contains more singular fields. The space of smooth fields is usually a set of measure zero with respect to the natural measure on this larger space. As we saw in Section 2.1, the classical configuration space $\mathcal{A}$ of general relativity can be taken to consist of smooth $\mathrm{SU}(2)$ connections on the spatial 3-manifold $M$. Its completion $\overline{\mathcal{A}}$ consists of 'generalized' $\mathrm{SU}(2)$ connections $[8,9]$. Like a connection, a generalized connection $A$ describes parallel transport along paths: it assigns to each path $\eta$ a holonomy $A(\eta)$, which we may think of as an element of SU(2). However, with a generalized connection there is no requirement that this holonomy vary smoothly with the path. Consequently, the space $\overline{\mathcal{A}}$ is very large. However, in the natural topology, the classical configuration space $\mathcal{A}$ is densely embedded in $\overline{\mathcal{A}}$. $\overline{\mathcal{A}}$ is sometimes referred to as the 'quantum configuration space'. (For precise definitions, see Section 4.1.) 
There are several distinct ways of constructing the space $\overline{\mathcal{A}}$. To do geometry and measure theory on $\overline{\mathcal{A}}$, it is convenient to construct it as the projective limit of configuration spaces $\mathcal{A}_{\mathfrak{g}}$ of $\mathrm{SU}(2)$ lattice gauge theories associated with graphs $\mathfrak{g}$ embedded in $M[10,11,12] .4$ More precisely, we can proceed as follows. First consider any graph $\mathfrak{g}$ with finitely many vertices and edges analytically embedded in the 3 -manifold $M$. Define a 'connection on $\mathfrak{g}$ ' to be a map $A$ assigning an element $A(e) \in \mathrm{SU}(2)$ to each edge $e$ of $\mathfrak{g}$ equipped with an orientation, and such that: i) If $e_{1}, e_{2}, e_{1} e_{2}$ are all analytic, then $A\left(e_{1}\right) A\left(e_{2}\right)=$ $A\left(e_{2} e_{1}\right)$; and, ii) $A\left(e^{-1}\right)=A(e)^{-1}$ when $e^{-1}$ is $e$ equipped with the opposite orientation. The group element $A(e)$ may be thought of as the holonomy of $A$ along the edge $e$. Let $\mathcal{A}_{\mathfrak{g}}$ be the space of connections on the graph $\mathfrak{g}$. $\mathcal{A}_{\mathfrak{g}}$ is naturally endowed with the structure of a finitedimensional compact manifold, diffeomorphic to a product of copies of $\mathrm{SU}(2)$, one for each edge of $\mathfrak{g}$. It can be thought of as the configuration space of the $\mathrm{SU}(2)$ lattice gauge theory associated with the graph $\mathfrak{g}$.

Next, note that the set of graphs in $M$ is naturally equipped with a partial ordering where $\mathfrak{g} \leq \mathfrak{g}^{\prime}$ if $\mathfrak{g}^{\prime}$ is obtained from $\mathfrak{g}$ by subdividing the edges of $\mathfrak{g}$ and/or adding new edges. Moreover, when $\mathfrak{g} \leq \mathfrak{g}^{\prime}$ there is an obvious projection

$$
p_{\mathfrak{g}, \mathfrak{g}^{\prime}}: \mathcal{A}_{\mathfrak{g}^{\prime}} \rightarrow \mathcal{A}_{\mathfrak{g}} .
$$

Since there is no 'largest' graph in $M$, the family of spaces $\mathcal{A}_{\mathfrak{g}}$ does not contain a largest space from which one can project to any other space in the family. However, as is well known, one can construct such a space, called the 'projective limit' of the spaces $\mathcal{A}_{\mathfrak{g}}$. This projective limit is precisely our quantum configuration space $\overrightarrow{\mathcal{A}}$. By definition of the projective limit, there are natural projections

$$
p_{\mathfrak{g}}: \overline{\mathcal{A}} \rightarrow \mathcal{A}_{\mathfrak{g}}
$$

for every graph $\mathfrak{g}$. Moreover, these are consistent with the family of projections $p_{\mathfrak{g}, \mathfrak{g}^{\prime}}$, in the sense that

$$
p_{\mathfrak{g}}=p_{\mathfrak{g}, \mathfrak{g} \prime} p_{\mathfrak{g}^{\prime}}
$$

\footnotetext{
${ }^{4}$ Note that, in spite of the similarity of an intermediate stage of our construction with lattice gauge theory, we do not follow the traditional strategy of first working with lattices and then taking the continuum limit by letting the edge length go to zero. Rather, we work with the continuum theory from the very beginning; lattice configuration spaces $\mathcal{A}_{\mathfrak{g}}$ only serve to provide useful approximations to the full continuum quantum configuration space $\overline{\mathcal{A}}$.
} 
for all $\mathfrak{g}, \mathfrak{g}^{\prime}$ with $\mathfrak{g} \leq \mathfrak{g}^{\prime}$.

As noted above, the space $\overline{\mathcal{A}}$ of generalized connections is very large. At first it may even seem mathematically uncontrollable. However, the projective limit construction endows it with a rich structure [12]. The basic idea is to first note that each space $\mathcal{A}_{\mathfrak{g}}$ is a equipped with an interesting topology, measure and geometry, and then to induce these structures on $\overline{\mathcal{A}}$ through the projection maps $p_{\mathfrak{g}}$. More precisely, there is a one-to-one correspondence between 'consistent sets' of structures on the family of finite-dimensional manifolds $\mathcal{A}_{\mathfrak{g}}$ and structures on $\overline{\mathcal{A}}$.

Let us illustrate this relation through some examples. Note first that the projection map $p_{\mathfrak{g}}$ enables us to pull back functions from any space $\mathcal{A}_{\mathfrak{g}}$ to $\overline{\mathcal{A}}$. Denote by Cyl the space of functions on $\overline{\mathcal{A}}$ obtained by pulling back $C^{\infty}$ functions on $\mathcal{A}_{\mathfrak{g}}$ for all graphs $\mathfrak{g}$. We will refer to the elements of $\mathrm{Cyl}$ as 'cylinder functions'. They play the role of smooth functions on $\overline{\mathcal{A}}$. Since $\mathcal{A}_{\mathfrak{g}}$ is a finite-dimensional manifold, the space of $C^{\infty}$ functions on any one $\mathcal{A}_{\mathfrak{g}}$ is relatively small and fully manageable. Yet, since in the construction of Cyl we allow $\mathfrak{g}$ to vary over all possible graphs in $M$, the space Cyl is very large.

A second useful example is provided by measures. Since $\mathcal{A}_{\mathfrak{g}}$ is diffeomorphic to a product of copies of $\mathrm{SU}(2)$, one for each edge of $\mathfrak{g}$, the Haar measure on $\mathrm{SU}(2)$ naturally gives rise to a probability measure $\mu_{\mathfrak{g}}$ on each space $\mathcal{A}_{\mathfrak{g}}$, The measures $\mu_{\mathfrak{g}}$ form a consistent set in the sense that if $\mathfrak{g} \leq \mathfrak{g}^{\prime}$, then $\mu_{\mathfrak{g}}$ is the pushforward of $\mu_{\mathfrak{g}^{\prime}}$ under the projection from $\mathcal{A}_{\mathfrak{g}^{\prime}}$ to $\mathcal{A}_{\mathfrak{g}}$ :

$$
\mu_{\mathfrak{g}}=\left(p_{\mathfrak{g}, \mathfrak{g}^{\prime}}\right)_{*} \mu_{\mathfrak{g}^{\prime}}
$$

Therefore, there exists $[9,10,11]$ a unique probability measure $\mu$ on $\overline{\mathcal{A}}$ that projects down to all the measures $\mu_{\mathfrak{g}}$ :

$$
\mu_{\mathfrak{g}}=\left(p_{\mathfrak{g}}\right)_{*} \mu .
$$

This measure $\mu$ is called the 'uniform measure'. By construction, all functions in Cyl are integrable with respect $\mu$. Moreover, since we did not introduce any extra structure such as a background metric in its construction, $\mu$ is invariant under the natural action of gauge transformations and diffeomorphisms on $\overline{\mathcal{A}}$. As in more traditional quantum field theories, although the classical configuration space $\mathcal{A}$ is topologically dense in the quantum configuration space $\overline{\mathcal{A}}$, measuretheoretically it is sparse: $\mathcal{A}$ is contained in a measurable subset of $\overline{\mathcal{A}}$ 
with measure zero [13].

Using the uniform measure $\mu$, we can now define the Hilbert space $\mathcal{H}$ of kinematical states for quantum gravity:

$$
\mathcal{H}=L^{2}(\overline{\mathcal{A}})
$$

This state space is the quantum analog of the kinematical phase space $\mathcal{X}$. In both cases, one has to impose the Einstein constraint equations to extract physical states. In the classical theory, the space of kinematical states provide an arena for a precise formulation of the constraint equations which play a central role in the dynamics of the Einstein's theory. Moreover, much of our physical intuition regarding geometric quantities such as the spatial metric, areas and volumes, extrinsic curvature, holonomies and their relation to local curvature comes from this kinematical arena. One expects, with appropriate caveats, the situation to be similar in quantum theory.

It turns out that the space Cyl of cylinder functions is dense in $\mathcal{H}$. This fact turns out to be very useful in quantum geometry. In particular, often one can first define interesting operators with Cyl as their domain and then show that they are essentially self-adjoint, thus determining self-adjoint operators on $\mathcal{H}$. (Thus Cyl plays a role in quantum gravity rather similar to that played by $C^{\infty}$ functions of compact support in non-relativistic quantum mechanics.) This strategy greatly simplifies the task of defining geometrical operators on $\mathcal{H}$. For, since elements of $\mathrm{Cyl}$ are pullbacks of smooth functions on the spaces $\mathcal{A}_{\mathfrak{g}}$, geometrical operators can now be defined as consistent families of operators on the Hilbert spaces

$$
\mathcal{H}_{\mathfrak{g}}=L^{2}\left(\mathcal{A}_{\mathfrak{g}}\right) .
$$

Thus, technically, a field-theoretic problem is now reduced to a set of quantum-mechanical problems. A further simplification arises because one can introduce a convenient basis in each $\mathcal{H}_{\mathfrak{g}}$ using a suitable generalization of Penrose's spin networks [14, 15, 16, 17, 20, 23], as described in Section 4.2. This further reduces the quantum-mechanical problem of defining operators on $\mathcal{H}_{\mathfrak{g}}$ to that of defining operators on finite-dimensional Hilbert spaces, characteristic of spin systems.

We can now introduce the geometrical operators. On the classical phase space, information about the Riemannian geometry of the 3 - 
manifold is coded in the $\operatorname{Ad} P$-valued 2-forms $\Sigma$. Specifically, orthonormal triads $E$ of density weight one which determine the Riemannian 3 -metric $q_{a b}$ via equation (2) are given by:

$$
E_{i}^{a}=\gamma \eta^{a b c} \Sigma_{b c i}
$$

(Recall that for simplicity we are suppressing the superscript $\gamma$ on $\Sigma$.) Therefore, any Riemannian geometric quantity, such as the area of a surface or volume of a region, can be constructed from these 2-forms $\Sigma$. The basic object in quantum Riemannian geometry are the corresponding operators $\hat{\Sigma}$. Since $\Sigma$ is a 2 -form on $M$, from geometric considerations one would expect $\hat{\Sigma}$ to be a 2-dimensional operator-valued distribution on $\mathcal{H}$. In other words, one expects to have an operator $\hat{\Sigma}_{T, f}$ given a surface $T$ in $M$ and an $\operatorname{Ad} P$-valued test field $f$ on this surface. This expectation is borne out.

We begin by describing some useful operators on the Hilbert spaces $\mathcal{H}_{\mathfrak{g}}$. Suppose that $v$ is a vertex of $\mathfrak{g}$ and that $e$ is an edge of $\mathfrak{g}$ having $v$ as one of its endpoints. Since $\mathcal{A}_{\mathfrak{g}}$ is a product of copies of $\mathrm{SU}(2)$, one for each edge of $\mathfrak{g}$, the left- and right-invariant vector fields $L^{i}, R^{i}$ on $\mathrm{SU}(2)$ give operators $L^{i}(e), R^{i}(e)$ on the $C^{\infty}$ functions on $\mathcal{A}_{\mathfrak{g}}$. Using these we define

$$
J^{i}(e, v)= \begin{cases}i L^{i}(e) & \text { if the edge } e \text { is oriented to be outgoing at } v \\ i R^{i}(e) & \text { if the edge } e \text { is oriented to be ingoing at } v\end{cases}
$$

and introduce 'vertex operators'

$$
J^{i}(v)=\sum_{e} J^{i}(e, v)
$$

where the sum extends over all edges having $v$ as an endpoint. These angular momentum-like operators $J^{i}(v)$ have a natural geometrical interpretation: they are the three generators of the $\mathrm{SU}(2)$ gauge rotations at $v$.

We are now ready to introduce the triad operators $\hat{\Sigma}_{T, f}$. Let $\psi$ be a $C^{\infty}$ function on $\mathcal{A}_{\mathfrak{g}}$. For the purposes of this paper, it will suffice to assume that all edges of the graph $\mathfrak{g}$ lie 'above' $T$, although some may intersect $S$ from above. (The notion of 'above' and 'below' refer to the orientation of $M$ and $T$.) By subdividing the graph if necessary, we can assume without loss of generality that each intersection point between 
$\mathfrak{g}$ and and $T$ is a vertex of $\mathfrak{g}$. Then, given an $\operatorname{Ad} P$-valued function $f$ on $T$, the smeared triad operator is given by:

$$
\hat{\Sigma}_{T, f}\left[p_{\mathfrak{g}}^{*} \psi\right]=p_{\mathfrak{g}}^{*}\left[8 \pi \gamma \ell_{P}^{2} \sum_{v} f_{i}(v) J^{i}(v) \psi\right],
$$

where the sum is taken over vertices $v$ where $\mathfrak{g}$ intersects $T$. The general definition, without any restriction on $\mathfrak{g}$, is given in [20]. One can show that $\hat{\Sigma}_{T, f}$ is a well-defined operator on Cyl, which moreover is essentially self-adjoint. Note that the action of the smeared triad operator $\hat{\Sigma}_{T, f}$ is localized at intersections of the smearing surface $T$ and the graph $\mathfrak{g}$ associated with the state $\psi$. Furthermore, the action is very natural, defined by the right- and left-invariant vector fields on $\mathrm{SU}(2)$. As with the construction of the Hilbert space of states, the definition of $\hat{\Sigma}_{T, f}$ does not refer to any background structure. The operator is therefore covariant under gauge transformations and diffeomorphisms of space.

Classically, geometric quantities such as areas of surfaces and volumes of regions are functions of the triads. Therefore, it is natural to construct the corresponding quantum operators by first expressing the classical quantities in terms of triads and then replacing the triads $\Sigma$ by operators $\hat{\Sigma}$. However, the functional form of these quantities can be quite complicated. Indeed, already formula (4) for the area of a surface is non-polynomial in the triads $\Sigma$. Nonetheless, somewhat surprisingly, the corresponding quantum operators can be constructed via suitable regularization [12]. The resulting length [22], area [19, 20] and volume $[19,21]$ operators are again covariant with respect to spatial diffeomorphisms.

In this paper, we will need only the area operator. Let us therefore focus on that case. As we saw in Section 2.1, given an oriented surface $T$ embedded in $M$, its area (4) defines a function $A_{T}$ on the classical phase space $\mathcal{X}$. The action of the corresponding quantum operator $\hat{A}_{T}$ is given by:

$$
\hat{A}_{T}\left[p_{\mathfrak{g}}^{*} \psi\right]=p_{\mathfrak{g}}^{*}\left[8 \pi \gamma \ell_{P}^{2} \sum_{v} \sqrt{J^{i}(v) J^{j}(v) k_{i j}} \psi\right] .
$$

where, for simplicity, we have again restricted ourselves to the case in which all edges of $\mathfrak{g}$ lie above $T$. (The square-root is well-defined 
because the operator in the parenthesis is a positive definite, essentially self-adjoint operator on $\mathcal{H}_{\mathfrak{g}}$.)

With Cyl as its domain, $\hat{A}_{T}$ is an essentially self-adjoint, positive definite operator on $\mathcal{H}$. Furthermore, it has some physically striking properties. First, all its eigenvalues are known in a closed form [12] and they are all discrete multiples of $\ell_{P}^{2}$, the square of the Planck length. Thus, at the Planck scale, the continuum picture breaks down in a precise sense. For any surface $T$, the smallest eigenvalue of $\hat{A}_{T}$ is of course zero. It turns out that the area gap - the value of the first non-zero eigenvalue - depends on the topology of $T$. Finally, the 'level spacing' - i.e., the difference between the consecutive eigenvaluesgoes to zero rapidly, as the exponential of the square-root of the area (in Planck units). Consequently, the continuum limit is reached very rapidly.

Since Cyl is dense in $\mathcal{H}$, heuristically a 'typical' state $\psi$ is associated with a graph $\mathfrak{g}$. Note that such functions $\psi(A)$ depend only on the action of the generalized connection $A$ on the edges of the graph $\mathfrak{g}$. In particular, in such states the area assigned to a surface not intersecting $\mathfrak{g}$ must vanish identically. More generally, these states represent excitations of geometry only along the graph $\mathfrak{g}$. In this sense, typical excitations of quantum geometry are one-dimensional, like a polymer. Therefore one says that in non-perturbative quantum gravity, space has a 'polymer geometry'.

Finally, as mentioned above, elements of $\mathcal{H}$ only represent 'kinematical states'. Nonetheless, just as the full phase space $\mathcal{X}$ plays an important role in the classical Hamiltonian framework, the structure provided by $\mathcal{H}$ is important to the quantum theory. In particular, this structure provides the tools that are needed to define the quantum constraint operators. Physical states of non-perturbative quantum gravity are annihilated by these operators. Because of the geometrical nature of the Gauss and diffeomorphism constraints, the corresponding operators are essentially unambiguous $[18,23,24]$. For the Hamiltonian constraint, such geometrical guidelines are not yet known. Nonetheless, specific proposals for the corresponding operators have been made (see in particular [27, 28]) and are currently being analyzed from various angles. Fortunately, the analysis of this paper depends only on some general assumptions on the solutions to the quantum Hamiltonian con- 
straint (since the lapse function smearing this constraint has to vanish on the horizon). Therefore, our results are largely insensitive to the detailed form that the final 'correct' constraint operator will have.

\section{The Quantization Strategy}

The quantization procedure used to handle the boundary conditions at the horizon and the relevant parts of the quantum Einstein equations is technically quite subtle. In particular, it requires a careful treatment of a number of delicate features of the U(1) Chern-Simons theory. These issues are discussed in detail in the next two sections. In this section, we will sketch the general strategy to orient the reader and to provide the overall logic behind the detailed, technical treatment that follows.

Recall from Section 2.1 that the volume and surface degrees of freedom cannot be separated in the classical theory: Since the horizon $S$ is the inner boundary of the spatial 3-manifold $M$, all fields on $S$ are determined by fields in the interior of $M$ by continuity. However, as we saw in Section 2.2, in the quantum theory the fields describing geometry become discontinuous in a certain precise sense, so the fields on $S$ are no longer determined by fields in $M$; in this case there are independent degrees of freedom living on the boundary. These surface degrees of freedom dictate the quantum geometry of the horizon and account for black hole entropy in our approach.

More precisely, since the holonomies of generalized connections along paths that lie on $S$ are quite independent from their holonomies along paths in the interior of $M$, the quantum configuration space $\overline{\mathcal{A}}$ is now given by a product $\overline{\mathcal{A}}=\overline{\mathcal{A}}_{V} \times \overline{\mathcal{A}}_{S}$ of the quantum configuration space associated with the interior of $M$ and that associated with the horizon $S$. Therefore, it is natural to begin with the product $\mathcal{H}_{V} \otimes \mathcal{H}_{S}$ of Hilbert spaces consisting of suitable functions on $\overline{\mathcal{A}}_{V}$ and $\overline{\mathcal{A}}_{S}$. The construction of volume states closely follows the procedure outlined in Section 2.2. Thus, there is a uniform measure $\mu_{V}$ on $\overline{\mathcal{A}}_{V}$ which enables one to construct $L^{2}\left(\overline{\mathcal{A}}_{V}\right)$. We could take this space as the volume Hilbert space. However, to avoid a proliferation of spaces and symbols, it is more convenient to impose at this stage the technically trivial part of the quantum Gauss constraint so that we can focus only on the 
nontrivial part later. Let $\mathcal{G}_{V}$ denote the space of all (not necessarily continuous) gauge transformations of $P$ that are the identity on $S$. We will take the volume Hilbert space $\mathcal{H}_{V}$ to be the subspace of $L^{2}\left(\overline{\mathcal{A}}_{V}\right)$ consisting of vectors that are invariant under $\mathcal{G}_{V}$.

The form of the symplectic structure (7) suggests that the surface Hilbert space $\mathcal{H}_{S}$ should be closely related to the Hilbert space of quantum states of Chern-Simons theory. To construct this space, let us first recall that, even at the kinematical level, fields $(A, \Sigma)$ in the phase space $\mathcal{X}$ have to satisfy the boundary condition (6) which arises because $S$ is the intersection of an isolated horizon with the spatial manifold $M$. This boundary condition must be incorporated also in the quantum theory. The heuristic idea is to quantize equation (6) by replacing $F$ and $\underline{\Sigma} \cdot r$ by the corresponding operators. By imposing this condition as an operator equation, physically we are allowing the triad as well as the curvature to fluctuate at the horizon but asking that they do so in tandem.

Now, since $F$ is the curvature of the surface connection $W$, one expects the field operator $\hat{F}$ to act on the surface Hilbert space $\mathcal{H}_{S}$ while, as we saw in Section 2.2 , the operator $\hat{\Sigma}$ acts on $\mathcal{H}_{V}$. Thus the quantum version of the horizon boundary condition (6) imposes a relation between the surface and volume states. One would naively expect it to constrain states $\Psi$ in $\mathcal{H}_{V} \otimes \mathcal{H}_{S}$ via

$$
(1 \otimes \hat{F}) \Psi=\left(-\frac{2 \pi \gamma}{a_{0}} \underline{\hat{\Sigma}} \cdot r \otimes 1\right) \Psi .
$$

However, it turns out that, because of certain subtleties associated with the quantum Chern-Simons theory (discussed in Sections 4.3.2 and 5.1.2), it is only the 'exponentiated version' $\exp (i \hat{F})$ of $\hat{F}$ that is well-defined on $\mathcal{H}_{S}$. Therefore, in place of (11), we are led to impose

$$
(1 \otimes \exp (i \hat{F})) \Psi=\left(\exp \left(-i \frac{2 \pi \gamma}{a_{0}} \underline{\underline{\Sigma}} \cdot r\right) \otimes 1\right) \Psi
$$

We call this the 'quantum boundary condition': Only those elements of $\mathcal{H}_{V} \otimes \mathcal{H}_{S}$ that satisfy this boundary condition can qualify as kinematical quantum states of our system.

The structure of this equation implies that we can obtain a basis $\Psi_{V} \otimes \Psi_{S}$ of solutions such that $\Psi_{V}$ and $\Psi_{S}$ are eigenstates of $\underline{\hat{\Sigma}} \cdot r$ and 
$\exp (i \hat{F})$ respectively, with

$$
\Psi_{V} \otimes \exp (i \hat{F}) \Psi_{S}=\exp \left(-i \frac{2 \pi \gamma}{a_{0}} \underline{\hat{\Sigma}} \cdot r\right) \Psi_{V} \otimes \Psi_{S}
$$

Now, all the eigenstates of $\underline{\hat{\Sigma}} \cdot r$ are known [20]. They satisfy

$$
(\underline{\hat{\Sigma}} \cdot r) \Psi_{V}=8 \pi \ell_{P}^{2} \sum_{i=1}^{n} m_{i} \delta^{2}\left(x, p_{i}\right) \eta \Psi_{V}
$$

where

$$
\mathcal{P}=\left\{p_{1}, \ldots, p_{n},\right\}
$$

is some finite set of points on $S, m_{i}$ are spins (i.e., half-integers) labelling these points, $\delta^{2}$ is the delta distribution on $S$, and $\eta$ the LeviCivita density on $S$. This fact allows a useful decomposition of the volume Hilbert space $\mathcal{H}_{V}$, and also leads to a precise construction of the surface Hilbert space $\mathcal{H}_{S}$.

Let us first consider $\mathcal{H}_{V}$. From our discussion in Section 2.2 it follows that the states in $\mathcal{H}_{V}$ satisfying equation (14) are cylinder functions based on graphs in $M$ whose edges have ends at the horizon at the points $\mathcal{P}$. If we let $\mathcal{H}_{V}^{\mathcal{P}, m}$ be the space of all states satisfying equation (14), then

$$
\mathcal{H}_{V}=\bigoplus_{\mathcal{P}, m} \mathcal{H}_{V}^{\mathcal{P}, m}
$$

where $\mathcal{P}$ ranges over all finite sets of points on $S$ and $m$ ranges over all ways of labelling these points with nonzero spins. This decomposition will be useful for solving the quantum boundary condition.

Let us now turn to the construction of the surface Hilbert space $\mathcal{H}_{S}$. Since classically the pullback of the connection $A$ to $S$ is determined by the $\mathrm{U}(1)$ connection $W$, it is natural to write the surface states $\Psi_{S}$ as a function of a generalized $\mathrm{U}(1)$ connection on $S$. Then (13) implies that $\Psi_{S}$ has support only on generalized U(1) connections that are flat everywhere except at finitely many points $p_{i}$ where the polymer geometry excitations in the bulk puncture $S$. Let us fix a set of points $\mathcal{P}$ and denote the space of such generalized connections by $\mathcal{A}^{\mathcal{P}}$ (for a precise definition of $\mathcal{A}^{\mathcal{P}}$, see Section 4.3.1). As with the volume Hilbert space, it is convenient to incorporate the technically trivial part of quantum Einstein's equations already in the definition of $\mathcal{H}_{S}$. Therefore, let us quotient $\mathcal{A}^{\mathcal{P}}$ by the action of the group $\mathcal{G}^{\mathcal{P}}$ of $\mathrm{U}(1)$ gauge 
transformations that are identity at the punctures $p_{i}$ and of the group $\mathcal{D}^{\mathcal{P}}$ consisting of diffeomorphisms of $S$ that fix each of the punctures $p_{i}$ together with certain structure needed for quantization. (For precise definitions of these groups, see Section 4.3.1.) One can show that the resulting space

$$
\mathcal{X}^{\mathcal{P}}=\mathcal{A}^{\mathcal{P}} /\left(\mathcal{G}^{\mathcal{P}} \rtimes \mathcal{D}^{\mathcal{P}}\right)
$$

is equipped with the Chern-Simons symplectic structure coming from the surface term in equation (7). Therefore, $\mathcal{X}^{\mathcal{P}}$ can be thought of as the phase space of the surface degrees of freedom associated with the set $\mathcal{P}$ of punctures. Denote by $\mathcal{H}_{S}^{\mathcal{P}}$ the Hilbert space obtained by geometric quantization of $\mathcal{X}^{\mathcal{P}}$. Then, the total surface Hilbert space $\mathcal{H}_{S}$ can be constructed as a direct limit of the spaces $\mathcal{H}_{S}^{\mathcal{P}}$ as the punctures $\mathcal{P}$ range over all finite subsets of $S$.

One can show that $\mathcal{X}^{\mathcal{P}}$ is isomorphic with a $2(n-1)$-dimensional torus:

$$
\mathcal{X}^{\mathcal{P}}=\mathbb{C}^{n-1} / \Lambda
$$

for the lattice $\Lambda=(2 \pi \mathbb{Z})^{2(n-1)}$. Therefore, to quantize $\mathcal{X}^{\mathcal{P}}$, one can begin with the space of holomorphic functions on $\mathbb{C}^{n-1}$ as in the Bargmann-Segal representation of the canonical commutation relations [29, $30]$ and then select only those states that are invariant under the discrete subgroup defined by $\Lambda$. These are the so-called 'theta functions', studied extensively since the 1800 s. Now, as is well known, the translation group on $\mathbb{C}^{n}$ acts only projectively in the Bargmann-Segal representation because, if we set $z=q+i p$, the translations in the $q$ directions fail to commute with the translations in the $p$ directions. As a result, nontrivial states that are invariant under the action of $\Lambda$ exist if and only if the constant

$$
k=\frac{a_{0}}{4 \pi \gamma \ell_{P}^{2}}
$$

is an integer. (The combination on the right comes from the coefficient in front of the surface term in the symplectic structure (7) which determines the phase factor in the projective representation.) This is the familiar 'prequantization condition', and $k$ is called the 'level' of the Chern-Simons theory. Next, associated with any small loop $\eta_{i}$ winding once around the puncture $p_{i}$ there is an operator $\hat{h}_{i}$ on $\mathcal{H}_{S}^{\mathcal{P}}$ which measures the holonomy around this loop. It turns out that eigenstates of these operators provide a basis $\psi_{\mathcal{P}, a}$ of the surface Hilbert space, where 
$\mathcal{P}$ ranges over all finite sets of points on the horizon and $a$ ranges over all ways of labelling these points by nonzero elements $a_{i}$ of $\mathbb{Z}_{k}$ (the group of integers modulo $k$ ), satisfying:

$$
a_{1}+\cdots+a_{n}=0 \text {. }
$$

We have

$$
\hat{h}_{i} \Psi_{\mathcal{P}, a}=e^{\frac{2 \pi i a_{i}}{k}} \Psi_{\mathcal{P}, a},
$$

so heuristically the state $\psi_{\mathcal{P}, a}$ describes a quantum geometry of the horizon in which the $\mathrm{U}(1)$ connection is flat except at the punctures $p_{i}$, with a holonomy of $\exp \left(2 \pi i a_{i} / k\right)$ around the $i$ th puncture. In short, the horizon is flat except at the punctures, where it has conical singularities with quantized angle deficits. Note that this quantization is a direct consequence of the prequantization condition on $k$. Thus, there is an interesting intertwining of quantum geometry and Chern-Simons theory.

The geometrical meaning of equation (16) is clarified if we work with the $\mathrm{SO}(2)$ 'Levi-Civita' connection instead of the $\mathrm{U}(1)$ connection. In these terms, the angle deficit at the $i$ th puncture is $4 \pi a_{i} / k$. However, since $a_{i}$ is only defined modulo $k$, these angle deficits are only defined modulo $4 \pi$, just as one would expect, given that our approach to quantum geometry is based on parallel transport of spinors. Equation (16) says that the sum of these angle deficits vanishes modulo $4 \pi$. This is a quantum analogue of the Gauss-Bonnet theorem, which says that for any metric on the 2-sphere, the integral of the scalar curvature equals $4 \pi$.

So far, we have discussed the structure of the volume and surface Hilbert spaces, $\mathcal{H}_{V}$ and $\mathcal{H}_{S}$, whose construction was motivated by the quantum boundary condition (13). Now that we have specific Hilbert spaces on hand, we can impose this condition on $\mathcal{H}_{V} \otimes \mathcal{H}_{S}$ in a precise fashion and explore its consequences. Note first that a state $\Psi_{V} \otimes \Psi_{S}$ can satisfy (13) if and only if the eigenvalue of $\exp \left(-i \frac{2 \pi \gamma}{a_{0}} \hat{\underline{\Sigma}} \cdot r\right)$ on $\Psi_{V}$ equals the eigenvalue of $\exp (i \hat{F})$ on $\Psi_{S}$. Now, the first of these is an operator on $\mathcal{H}_{V}$ defined in the quantum geometry framework, while the second is an operator on $\mathcal{H}_{S}$ constructed using Chern-Simons theory. A priori it is not at all obvious that the spectra of these two distinct operators have any overlap. If they do not, (13) would have no solutions and this approach to the quantum geometry of horizons and black hole entropy would not be viable. 
Now, it follows from the above discussion that each operator has a nontrivial action only at the punctures. By equation (14), at each puncture $p_{i}$ the eigenvalues of $\exp \left(-i \frac{2 \pi \gamma}{a_{0}} \underline{\underline{\Sigma}} \cdot r\right)$ are of the form

$$
\exp \left(-\frac{2 \pi i \gamma}{a_{0}}\left(8 \pi \ell_{P}^{2} m_{i}\right)\right)
$$

where $m_{i}$ is an half-integer. Similarly, equation (17) implies that the eigenvalues of $\exp (i \hat{F})$ at $p_{i}$ are of the form

$$
\exp \left(\frac{2 \pi i a_{i}}{k}\right)
$$

where $a_{i}$ is any integer mod $k$. (For details, see Sections 4.3.2 and 5.1.) Simple algebra using equation (15) shows that these spectra in fact coincide! The eigenvalues match when $2 m_{i}=-a_{i} \bmod k$. This is a striking example of the unexpected, detailed matching between classical general relativity (which dictates the horizon boundary condition), quantum geometry (which determines the action of $\underline{\underline{\Sigma}} \cdot r$ ) and quantum Chern-Simons theory (which determines $\exp (i \hat{F}))$.

With this background material at hand, we can now exhibit the space of solutions to the quantum boundary conditions, i.e., the kinematical Hilbert space: It is simply the subspace of $\mathcal{H}_{V} \otimes \mathcal{H}_{S}$ given by

$$
\mathcal{H}^{\mathrm{Kin}}=\bigoplus_{\mathcal{P}, m, a:} \mathcal{H}_{V}^{\mathcal{P}, m} \otimes \mathcal{H}_{S}^{\mathcal{P}, a}
$$

where $\mathcal{H}_{S}^{\mathcal{P}, a}$ is the one-dimensional Hilbert space spanned by the surface state $\psi_{\mathcal{P}, a}$ introduced above. As one might have expected, the volume states are correlated with the surface states at the punctures in a specific way.

Finally, one has to impose the quantum versions of Einstein constraints to extract physical states of our system. As noted in Section 2.1, there are three sets of constraints: the Gauss, diffeomorphism and Hamiltonian constraints. Since the 'lapse function' smearing the Hamiltonian constraint must vanish at the horizon, this constraint will not play an essential role in determining the quantum geometry of the horizon. Let us therefore concentrate on the other two.

Let us begin with the Gauss constraint which demands that physical states be gauge invariant. By construction, all elements of $\mathcal{H}_{V}^{\mathcal{P}} \otimes \mathcal{H}_{S}^{\mathcal{P}}$ are 
invariant under gauge transformations that are identity at the punctures. Therefore, it only remains to ensure gauge invariance at the punctures. Now, since the commutation relations on the surface Hilbert space are dictated by the Chern-Simons symplectic structure, gauge rotations on $\mathcal{H}_{S}^{\mathcal{P}}$ are implemented precisely by the surface operator in the quantum boundary condition (12). Similarly, on the volume Hilbert space, the operator $\hat{\Sigma} \cdot r$ generates $\mathrm{U}(1)$ gauge transformations on the horizon. As a consequence, the volume operator in (13) implements the action of $\mathrm{U}(1)$ gauge transformations at the punctures on $\mathcal{H}_{V}^{\mathcal{P}}$. Thus, the meaning of equation (13) turns out to be rather simple: it ensures that the volume and surface states are 'coupled' in precisely the correct way so that the total state is invariant under the allowed $U(1)$ internal rotations on the horizon. Thus, the quantum Gauss constraint is automatically satisfied on the Hilbert space $\mathcal{H}$.

Finally, let us consider the diffeomorphism constraint. This constraint simply implies that two states in the Hilbert space $\mathcal{H}$ should be regarded as equivalent if they are related by a diffeomorphism of $M$ that maps $S$ to itself. For the quantum geometry of the horizon, only the action of the diffeomorphisms on $S$ is relevant. This action is rather subtle because the construction of $\mathcal{H}_{S}^{\mathcal{P}}$ requires the introduction of an extra structure on $S$. Nonetheless, the final result is transparent and easy to state. Since any two sets $\mathcal{P}$ and $\mathcal{P}^{\prime}$ with the same number of punctures are related by a diffeomorphism, quantum geometries of the horizon compatible with $\mathcal{P}$ are physically indistinguishable with those compatible with $\mathcal{P}^{\prime}$. The 'locations' of punctures are irrelevant; what matters is only the number of punctures. This fact plays an important role in entropy calculation of Section 6.

\section{Kinematical Hilbert Spaces}

Using the overall strategy presented in Section 3 as a guideline we will now introduce the kinematical Hilbert spaces. In Section 4.1, we extend the theory of generalized connections to the case of a manifold with boundary. We simply describe the necessary results, because the proofs are similar to the case of manifolds without boundary. In Section 4.2 , we obtain the volume Hilbert space again by a simple extension of the framework of the case without boundary discussed in Section 2.2. 
In Section 4.3 we construct the surface Hilbert space using quantum Chern-Simons theory. This discussion is more detailed.

\subsection{Generalized Connections}

In Section 2.2 we introduced generalized connections on a manifold without boundary. The situation is a bit more complicated in our treatment of the black hole, due to the boundary conditions at the horizon $S$. However, the theory of generalized connections still forms the basis of our treatment and, as emphasized before, is in fact crucial for understanding the separation of volume and surface degrees of freedom that occurs. We shall show that the space $\overline{\mathcal{A}}$ of generalized connections can be written as a product of two spaces, $\overline{\mathcal{A}}_{V}$ and $\overline{\mathcal{A}}_{S}$, consisting of generalized connections 'in the volume' and 'on the surface' respectively. Starting with these spaces, in the next two sections we construct in detail the volume and surface Hilbert spaces for the quantum black hole along the lines outlined in Section 3.

For the sake of possible future generalizations, we proceed quite generally in this section, and specialize to the case at hand later. Suppose $X$ is a real-analytic manifold with boundary $\partial X$. We define a 'path in $X^{\prime}$ to be an equivalence class of analytic maps $\eta:[0,1] \rightarrow X$ with $\eta^{\prime}(t) \neq 0$, where we consider two such maps the same if they differ by a reparametrization, that is, an analytic orientation-preserving diffeomorphism of $[0,1]$. Given a path $\eta:[0,1] \rightarrow X$, we call $\eta(0)$ its 'source' and $\eta(1)$ its 'target'. If $x \in X$ is the source or target of $\eta$ we say $\eta$ is 'incident' to $x$. Given $\eta_{1}$ and $\eta_{2}$, if the target of $\eta_{1}$ equals the source of $\eta_{2}$ we let the product $\eta_{1} \eta_{2}$ be the path consisting of $\eta_{1}$ followed by $\eta_{2}$. Similarly, we let $\eta^{-1}$ be the path formed by reversing the orientation of the path $\eta$.

Suppose $P$ is a smooth principal $G$-bundle over $X$, with $G$ a compact connected Lie group. We fix a trivialization of $P$ at each point $x \in X$, so that we can think of parallel transport along any path in $X$ as an element of $G$. (We do not demand that this trivialization vary continuously with $x$.) We define a 'generalized connection on $P$ ' to be a map $A$ assigning to each path in $X$ an element of $G$, satisfying the following properties: 

1. $A\left(\eta_{1} \eta_{2}\right)=A\left(\eta_{2}\right) A\left(\eta_{1}\right)$
2. $A\left(\eta^{-1}\right)=A(\eta)^{-1}$

We think of the group element $A(\eta)$ as describing parallel transport from the source of $\eta$ to its target.

Let $\mathcal{A}$ be the space of smooth connections on $P$ and let $\overline{\mathcal{A}}$ be the space of generalized connections on $P$. There is a one-to-one map from $\mathcal{A}$ to $\overline{\mathcal{A}}$ that assigns to each smooth connection the generalized connection having the same holonomies along paths. This allows us to think of $\mathcal{A}$ as a subspace of $\overline{\mathcal{A}}$. There is a natural topology on $\overline{\mathcal{A}}$ for which this subspace is dense, so we may think of $\overline{\mathcal{A}}$ as a 'completion' of the space of smooth connections. A key advantage of working with this completion is that it possesses a natural measure, invariant under both diffeomorphisms and gauge transformations, called the 'uniform measure'.

The uniform measure $\mu$ on $\overline{\mathcal{A}}$ is defined just as in the case of a manifold without boundary $[9,11,16]$. In Section 2.2 , we introduced it as the projective limit of the family of measures $\mu_{\mathfrak{g}}$ on configuration spaces $\mathcal{A}_{\mathfrak{g}}$ associated with graphs $\mathfrak{g}$. We will now give an different but equivalent characterization which brings out how natural this measure is. Define an 'edge in $X$ ' to be a path in $X$ which restricts to an embedding of the interval $(0,1)$. Given a finite set of paths $\eta_{i}$ in $X$, we can always find a finite set of edges $e_{j}$ in $X$ such that:

(a) Each path $\eta_{i}$ is a product of finitely many edges $e_{j}$ and their inverses.

(b) Distinct edges $e_{j}$ intersect, if at all, only at their endpoints.

A finite set of edges with property (b) is called a 'graph in $X$ ', and we call the endpoints of these edges the 'vertices' of the graph. The uniform measure on $\overline{\mathcal{A}}$ is characterized by the following property: for any graph $\mathfrak{g}=\left\{e_{j}\right\}$ in $X$, the group elements $A\left(e_{j}\right)$ are independent $G$-valued random variables, each distributed according to normalized Haar measure on $G$. We denote this measure by $\mu$.

For applications to black holes we need a way of separating a generalized connection into two parts: the 'volume' part, which describes 
holonomies along paths having no subinterval lying in the boundary $\partial X$, and the 'surface' part, which describes holonomies along paths lying completely in $\partial X$. To do this, we define a 'path in the volume' to be one which has no subinterval lying in $\partial X$, and define a 'generalized connection in the volume' to be a map $A$ assigning to each path in the volume an element $A(\eta) \in G$, satisfying the properties 1 and 2 listed above. Similarly, we define a 'generalized connection on the surface' to be a map $A$ assigning to each path in $\partial X$ an element $A(\eta) \in G$ satisfying properties 1 and 2 .

Let $\overline{\mathcal{A}}_{V}$ denote the space of generalized connections in the volume, and let $\overline{\mathcal{A}}_{S}$ denote the space of generalized connections on the surface. A generalized connection on $P$ determines generalized connections in the volume and on the surface, so we have maps

$$
p_{V}: \overline{\mathcal{A}} \rightarrow \overline{\mathcal{A}}_{V}, \quad p_{S}: \overline{\mathcal{A}} \rightarrow \overline{\mathcal{A}}_{S}
$$

One can check that in fact

$$
\overline{\mathcal{A}}=\overline{\mathcal{A}}_{V} \times \overline{\mathcal{A}}_{S}
$$

with the above maps being the projections onto the two factors.

We can define 'uniform measures' on $\overline{\mathcal{A}}_{V}$ and $\overline{\mathcal{A}}_{S}$ by pushing $\mu$ forwards along the projections from $\overline{\mathcal{A}}$ to these two spaces:

$$
\mu_{V}=\left(p_{V}\right)_{*} \mu, \quad \mu_{S}=\left(p_{S}\right)_{*} \mu
$$

Moreover, one can check that, with their uniform measures, $\overline{\mathcal{A}}$ is equal as a measure space to the product $\overline{\mathcal{A}}_{V} \times \overline{\mathcal{A}}_{S}$. This boils down to the fact that with respect to the uniform measure, holonomies along collections of paths in the volume are independent (as random variables) from holonomies along collections of paths in the boundary $\partial X$.

\subsection{The Volume Hilbert Space}

Now let us focus attention on the case of physical interest: the 3manifold $M$ with the 2-sphere $S$ as its interior boundary. Here $P$ is the trivial $\mathrm{SU}(2)$ bundle over $M$, and we use a fixed trivialization to think of holonomies along paths in $M$ as group elements. 
Using the results of the previous section we define the space $\overline{\mathcal{A}}_{V}$ of generalized connections in the volume, and equip it with its uniform measure $\mu_{V}$. Using this measure one can define the Hilbert space $L^{2}\left(\overline{\mathcal{A}}_{V}\right)$. As in the case of manifolds without boundary $[15,16,17,18$, $20]$, one can introduce an explicit set of vectors spanning this space labelled by triples $\psi=(\mathfrak{g}, \rho, \nu)$ such that:

1. $\mathfrak{g}$ is a graph in the volume,

2. $\rho$ assigns to each edge $e$ of $\mathfrak{g}$ a nontrivial irreducible representation $\rho_{e}$ of $\mathrm{SU}(2)$,

3. $\nu$ assigns to each vertex $v$ of $\mathfrak{g}$ a vector $\nu_{v}$ in the tensor product of the representations $\rho_{e}$ labelling edges $e$ incident to $v$.

Here we say a graph $\mathfrak{g}$ in $M$ is 'in the volume' if all its edges have no subinterval lying in $S$.

From any such triple we get a function in $L^{2}\left(\overline{\mathcal{A}}_{V}\right)$, which we also call $\psi$, as follows:

$$
\psi(A)=\left\langle\bigotimes_{e} \rho_{e}(A(e)), \bigotimes_{v} \nu(v)\right\rangle .
$$

Here the first tensor product is taken over the edges of $\mathfrak{g}$, the second is taken over the vertices of $\mathfrak{g}$, and $\langle\cdot, \cdot\rangle$ denotes the natural pairing given by contraction of indices. If we let $\mathfrak{g}$ range over all graphs inside $M$, let $\rho$ range over all labellings of edges by nonzero spins, and let $\nu$ range over all labellings of vertices by vectors chosen from an orthonormal basis, we obtain states $\psi$ forming an orthonormal basis of $L^{2}\left(\overline{\mathcal{A}}_{V}\right)$.

Let $\mathcal{G}_{V}$ denote the group of (not necessarily continuous) gauge transformations of $P$ that equal the identity on $S$. Since gauge transformations act as unitary operators on $L^{2}\left(\overline{\mathcal{A}}_{V}\right)$, we may define the 'volume Hilbert space', $\mathcal{H}_{V}$, to be the subspace of $L^{2}\left(\overline{\mathcal{A}}_{V}\right)$ consisting of vectors invariant under the action of $\mathcal{G}_{V}$. Starting from the explicit description of $L^{2}\left(\overline{\mathcal{A}}_{V}\right)$ in the previous paragraph, one can prove that the volume Hilbert space is spanned by vectors $\psi$ corresponding to triples $(\mathfrak{g}, \rho, \iota)$ of the following form:

1. $\mathfrak{g}$ is a graph in the volume, 
2. $\rho$ assigns to each edge $e$ of $\mathfrak{g}$ a nontrivial irreducible representation $\rho_{e}$ of $\mathrm{SU}(2)$,

3. $\iota$ assigns to each vertex $v$ of $\mathfrak{g}$ a vector $\iota_{v}$ in the tensor product of the representations $\rho_{e}$ labelling edges $e$ incident to $v$, and if $v$ lies in the interior of $M, \iota_{v}$ must be invariant under the action of $\mathrm{SU}(2)$ on this tensor product.

We call such a triple an '(open) spin network'. We use the same symbol $\psi$ to denote a spin network and the vector in the volume Hilbert space that it determines. If we let $\mathfrak{g}$ range over all graphs in $M$, let $\rho$ range over all labellings of edges by nonzero spins, and let $\iota$ range over all labellings of vertices by vectors chosen from an orthonormal basis, we obtain states forming an orthonormal basis of $\mathcal{H}_{V}$.

Any spin network $\psi=(\mathfrak{g}, \rho, \iota)$ has a set of 'ends', namely the vertices of the graph $\mathfrak{g}$ that lie in $S$. For any finite $\mathcal{P}=\left\{p_{1}, \ldots, p_{n}\right\}$ of $S$, let $\mathcal{H}_{V}^{\mathcal{P}}$ be the subspace of the volume Hilbert space spanned by all open spin networks whose ends lie in the set $\mathcal{P}$. Note that since every open spin network lies in some space $\mathcal{H}_{V}^{\mathcal{P}}$, the volume Hilbert space is the closure of the union of all these spaces $\mathcal{H}_{V}^{\mathcal{P}}$. Also note that if $\mathcal{P} \subseteq \mathcal{P}^{\prime}$, then $\mathcal{H}_{V}^{\mathcal{P}} \subseteq \mathcal{H}_{V}^{\mathcal{P}^{\prime}}$. Technically, these two facts together say that the Hilbert space volume Hilbert space is the 'direct limit' of the Hilbert spaces $\mathcal{H}_{V}^{\mathcal{P}}$ :

$$
\mathcal{H}_{V}=\lim _{\mathcal{P}} \mathcal{H}_{V}^{\mathcal{P}}
$$

as $\mathcal{P}$ ranges over all finite subsets of $S$. In the next section, we shall give a similar description of the surface Hilbert space as a direct limit. These descriptions become important when we implement the quantum boundary condition in Section 5.1.

We conclude by describing an important operator on the volume Hilbert space, namely the horizon area operator. Construction of this operator follows the usual treatment in quantum geometry [19, 20], as reviewed in Section 2.2. We need only adapt it slightly to take into account the special role of the horizon.

Let $\mathcal{G}_{S}$ denote the group of all (not necessarily continuous) gauge transformations of the bundle $\left.P\right|_{S}$. This group acts on $\overline{\mathcal{A}}_{V}$ in a measurepreserving way so it acts as unitary operators on $L^{2}\left(\overline{\mathcal{A}}_{V}\right)$. Since these operators commute with the action of $\mathcal{G}_{V}$ on $L^{2}\left(\overline{\mathcal{A}}_{V}\right)$, they preserve 
the volume Hilbert space $\mathcal{H}_{V}$, which consists of the functions invariant under the action of $\mathcal{G}_{V}$. We thus obtain a unitary representation of $\mathcal{G}_{S}$ on $\mathcal{H}_{V}$.

In particular, if we pick any point $p \in S$, we obtain a unitary representation of $\mathrm{SU}(2)$ on $\mathcal{H}_{V}$ by considering gauge transformations in $\mathcal{G}_{S}$ that are the identity at every point except $p$. Let $J^{i}(p)$ stand for the self-adjoint infinitesimal generators of this representation, satisfying the usual angular momentum commutation relations, and let

$$
J(p) \cdot J(p)=k_{i j} J^{i}(p) J^{j}(p)
$$

be the corresponding Casimir. Then as described in equation (10) there is a self-adjoint operator $\hat{A}_{S}$ on the volume Hilbert space that measures the area of the horizon, given by

$$
\hat{A}_{S}=8 \pi \gamma \ell_{P}^{2} \sum_{p \in S} \sqrt{J(p) \cdot J(p)} .
$$

Note that although the sum is uncountable, only countably many terms give a nonzero contribution when this operator is applied to any state.

Suppose $\mathcal{P}$ is a finite subset of points in $S$ and $j=\left(j_{1}, \ldots, j_{n}\right)$ is a way of labelling each point $p_{i} \in \mathcal{P}$ with a nonzero spin (i.e. halfintegers) $j_{i}$. Then there is a subspace $\mathcal{H}_{V}^{\mathcal{P}, j}$ of the volume Hilbert space consisting of all vectors $\psi$ such that

$$
\left(J\left(p_{i}\right) \cdot J\left(p_{i}\right)\right) \psi=j_{i}\left(j_{i}+1\right) \psi
$$

for each point $p_{i}$ and

$$
(J(p) \cdot J(p)) \psi=0
$$

for all points $p$ other than the points $p_{i}$. Clearly $\mathcal{H}_{V}^{\mathcal{P}, j}$ is a subspace of $\mathcal{H}_{V}^{\mathcal{P}}$, since gauge transformations at any point $p \in S$ act trivially on states corresponding to spin networks that do not have $p$ as one of their ends. Moreover, we have a direct sum decomposition:

$$
\mathcal{H}_{V}=\bigoplus_{\mathcal{P}, j} \mathcal{H}_{V}^{\mathcal{P}, j}
$$

where $\mathcal{P}, j$ range over all finite sets of points in $S$ labelled by nonzero spins. This decomposition serves to diagonalize the area operator $\hat{A}_{S}$, 
since any state $\psi \in \mathcal{H}_{V}^{\mathcal{P}, j}$ is an eigenstate of this operator with eigenvalue given by:

$$
\hat{A}_{S} \psi=8 \pi \gamma \ell_{P}^{2} \sum_{i} \sqrt{j_{i}\left(j_{i}+1\right)} \psi
$$

where the sum is taken over all the spins labelling points in $\mathcal{P}$.

\subsection{The Surface Hilbert Space}

In this section we construct the surface Hilbert space $\mathcal{H}_{S}$ by geometrically quantizing the space of generalized connections on the 2-sphere $S$ while taking into account the quantum boundary condition (12) and the surface term in the symplectic structure (7). As discussed in Section 3, the first task is to construct, for each finite set $\mathcal{P}$ of points on $S$ the quantum phase space $\mathcal{X}^{\mathcal{P}}$ consisting of generalized connections that are flat everywhere on $S$ except at these points. This will be carried out in the first subsection. In the second subsection, we will quantize this phase space. As noted in Section 3, technical subtleties of this quantization have an important effect on the quantum geometry of the horizon.

\subsubsection{The phase space $\mathcal{X}^{\mathcal{P}}$}

Recall that the $\mathrm{SU}(2)$ bundle $\left.P\right|_{S}$ has a $\mathrm{U}(1)$ sub-bundle $Q$ which is isomorphic to the spin bundle of the sphere $S$. As in Section 4.1, we define a 'generalized connection on the surface' to be a map $W$ assigning to each path $\eta$ in $S$ a holonomy $W(\eta) \in \mathrm{SU}(2)$ in a consistent manner. This definition makes use of a trivialization of $\left.P\right|_{S}$ over each point of $S$. If we wish to avoid this, we can alternatively think of $W(\eta)$ as a 'transporter': a map from the fiber $P_{\eta(0)}$ to the fiber $P_{\eta(1)}$. We then call $W$ a 'generalized $\mathrm{U}(1)$ connection' if for each path $\eta, W(\eta)$ maps $Q_{\eta(0)}$ to $Q_{\eta(1)}$. We may also think of a generalized U(1) connection as a generalized connection on the bundle $Q$.

Next, let $\mathcal{P}=\left\{p_{1}, \ldots, p_{n}\right\}$ be a finite set of points in $S$. We call these points 'punctures'. We say that a generalized $U(1)$ connection $W$ is 'flat except at the punctures' if it assigns the same holonomies to paths as a connection $W_{0}$ on $Q$ with the following properties: 
1. $W_{0}$ is flat on $S-\mathcal{P}$.

2. For some neighborhood $U_{i}$ of each puncture $p_{i}$, some smooth trivialization of $Q$ over $U_{i}$, and some analytic coordinate system $(x, y)$ on $U_{i}$ for which $p_{i}$ has the coordinates $(a, b), W_{0}$ has the following form:

$$
W_{0}=W_{1}+c \frac{(x-a) d y-(y-b) d x}{(x-a)^{2}+(y-b)^{2}}
$$

on $U_{i}-\left\{p_{i}\right\}$, where $c \in \mathbb{R}$ and $W_{1}$ is a bounded smooth 1-form on $U_{i}-\left\{p_{i}\right\}$.

This definition requires some comment. With condition 1 we demand that $W$ be flat away from the punctures, and with condition 2 we demand that at each puncture it has the usual sort of singularity produced, say, in Maxwell theory by a magnetic flux line intersecting $S$ transversely at that point. Since the connection $W_{0}$ is singular at the punctures we must actually check that it assigns a well-defined holonomy to paths going through these points. It suffices to consider the case where $e:[0,1] \rightarrow S$ is an edge lying completely within the neighborhood of $p_{i}$ where $W_{0}$ has the stated form, and where $e(t)=p_{i}$ for $t=0$ but for no other values of $t$. Using polar coordinates $(r, \theta)$ centered at the point $p_{i}$, a naive calculation shows that

$$
\int_{e} W_{0}=\int_{e} W_{1}+c \int_{0}^{1} \frac{d \theta(e(t))}{d t} d t .
$$

The first integral on the right-hand side is well-defined because $W_{1}$ is smooth and bounded on $U_{i}-\left\{p_{i}\right\}$. Similarly, even though $\theta$ is multivalued and $\theta(e(t))$ is undefined at $t=0$, the second integral on the right-hand side is well-defined, because using the fact that $e$ is analytic, one can show that $d \theta(e(t)) / d t$ is well-defined and continuous for $t>0$ and has a finite limit as $t \downarrow 0$. We therefore use this formula to define the holonomy of $W_{0}$ along the edge $e$. It is simple to verify that the two conditions of Section 4.1, required of generalized connections, are satisfied.

Let $\mathcal{A}^{\mathcal{P}}$ be the space of generalized $\mathrm{U}(1)$ connections that are flat except at the punctures. Let $\mathcal{G}^{\mathcal{P}}$ be the group of (not necessarily continuous) gauge transformations of $Q$ that equal the identity at the punctures. Fix a ray in the tangent space of each puncture, and let $\mathcal{D}^{\mathcal{P}}$ be the identity component of the group of orientation-preserving 
analytic diffeomorphisms that fix each puncture together with these rays. The semidirect product $\mathcal{G}^{\mathcal{P}} \rtimes \mathcal{D}^{\mathcal{P}}$ is a subgroup of the group of all automorphisms of the bundle $Q$. Since the space $\mathcal{A}^{\mathcal{P}}$ is defined in a gauge- and diffeomorphism-covariant way, the group $\mathcal{G}^{\mathcal{P}} \rtimes \mathcal{D}^{\mathcal{P}}$ acts on $\mathcal{A}^{\mathcal{P}}$. The quantum Einstein equations require that we treat this action as gauge. We are therefore led to consider the quotient space $\mathcal{X}^{\mathcal{P}}=\mathcal{A}^{\mathcal{P}} /\left(\mathcal{G}^{\mathcal{P}} \rtimes \mathcal{D}^{\mathcal{P}}\right)$.

Our next goal is to describe $\mathcal{X}^{\mathcal{P}}$.

Theorem 1. The space $\mathcal{X}^{\mathcal{P}}$ is diffeomorphic to a $2(n-1)$-dimensional torus.

Proof. We begin by studying the consequences of conditions 1 and 2 above. In what follows, we shall not distinguish between a generalized $\mathrm{U}(1)$ connection $W$ that is flat except at the punctures and a distributional connection $W_{0}$ assigning the same holonomies to paths and satisfying conditions 1 and 2. Also, if condition 2 holds for some realanalytic coordinate system in a neighborhood of the puncture $p_{i}$ and some smooth trivialization of $Q$ in this neighborhood, we say that $W$ has a 'singularity of standard form' at $p_{i}$ with respect to this coordinate system and trivialization.

Suppose that $W$ has a singularity of standard form at $p$ with respect to some local coordinate system $(x, y)$ and some local trivialization of $Q$. Then we can ask if this is also true with respect to some other local coordinate system and/or trivialization. If we change the trivialization, this adds a smooth closed 1-form to $W$, so $W$ still has a singularity of standard form at $p$ with respect to the new trivialization. If we change the coordinate system, $W$ may not have a singularity of standard form with respect to the new coordinates. However, it will if the new coordinates $\left(x^{\prime}, y^{\prime}\right)$ satisfy

$$
d x^{2}+d y^{\prime 2}=c\left(d x^{2}+d y^{2}\right)
$$

at the point $p$, for some scale factor $c$. This can be seen by an explicit calculation. It follows that having a singularity of standard form at $p$, which a priori depends on a choice of a local coordinate system and a local trivialization of $Q$, in fact depends only on a choice of a conformal structure at $p$, i.e., a metric modulo scale factor on the tangent space at $p$. 
Now, choose an open disc $U$ containing all the punctures, a trivialization of $Q$ over $U$, and an analytic coordinate system $(x, y)$ on $U$ for which each puncture $p_{i}$ has the coordinates $(i, 0)$ and the fixed ray in the tangent space of $p_{i}$ is spanned by the tangent vector $\partial_{y}$. Suppose that $W$ is a generalized $\mathrm{U}(1)$ connection flat except at the punctures. Then it has singularities of standard form at each puncture $p_{i}$ with respect to some conformal structure at $p_{i}$. However, we can always find a transformation in $\mathcal{D}^{\mathcal{P}}$ that maps all of these conformal structures at the punctures to the conformal structure corresponding to the metric $d x^{2}+d y^{2}$. To see this, first note that one can map any conformal structure at $p_{i}$ to any other while leaving a specified tangent vector fixed. Thus there exists a smooth vector field $v$ generating a flow $F_{t}$ on $S$ that fixes the punctures together with the tangent vectors $\partial_{y}$ at the punctures, and such that $F_{1}$ maps the conformal structures at the punctures to the conformal structure corresponding to $d x^{2}+d y^{2}$. Choose a real-analytic vector field $w$ that agrees with $v$ to first order at the punctures, and let $G_{t}$ be the flow generated by $w$. The diffeomorphism $G_{1}$ will then have all the desired properties.

It follows that for any point $[W] \in \mathcal{X}^{\mathcal{P}}$, we can find a representative $W \in \mathcal{A}^{\mathcal{P}}$ that has singularities of standard form at the points $p_{i}$, all with respect to the chosen coordinates and trivialization of $Q$ over $U$. Concretely, this means that on $U-\mathcal{P}$ we have

$$
W=W_{1}+\sum_{i=1}^{n} c_{i} \frac{(x-i) d y-y d x}{(x-i)^{2}+y^{2}}
$$

where $W_{1}$ is a bounded smooth 1-form on $U-\mathcal{P}$. Since $W$ is flat except at the punctures, $W_{1}$ must be closed. Note that the constants $c_{i}$ are not independent: they must sum to zero modulo $2 \pi$, because the holonomy of $W$ around a loop enclosing all the punctures must be trivial.

In fact, for any point in $\mathcal{X}^{\mathcal{P}}$ we can find a representative of a very special form. To describe this special form, we first choose real-analytic paths $\gamma_{1}, \ldots, \gamma_{n-1}$ and $\eta_{1}, \ldots, \eta_{n-1}$ as shown in Figure 4 . Note that $\gamma_{i}$ is a semicircular arc in the upper half plane going from $p_{i}$ to $p_{n}$, while $\eta_{i}$ is a circular loop going around $p_{i}$ once counterclockwise and not containing any of the other punctures. Next, for $1 \leq i \leq n-1$, we choose smooth 1 -forms $X_{i}, Y_{i}$ on $S-\mathcal{P}$ with the following properties: 


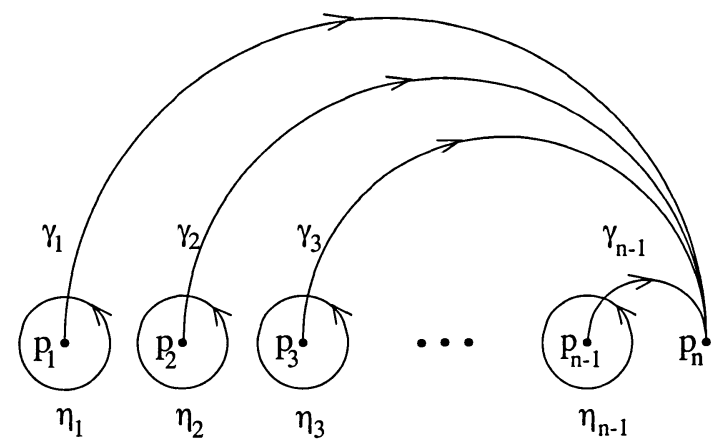

Figure 4: Choice of paths.

(a) $X_{i}$ satisfies

$$
\int_{\gamma_{j}} X_{i}=\delta_{i j}, \quad \oint_{\eta_{j}} X_{i}=0
$$

(b) $Y_{i}$ satisfies

$$
\oint_{\eta_{j}} Y_{i}=\delta_{i j}, \quad \int_{\gamma_{j}} X_{i}=0
$$

(c) $X_{i}$ and $Y_{i}$ satisfy

$$
\int_{S^{2}} X_{i} \wedge X_{j}=0, \quad \int_{S^{2}} Y_{i} \wedge Y_{j}=0, \quad \int_{S^{2}} X_{i} \wedge Y_{j}=\delta_{i j}
$$

(d) For any real constants $x_{i}, y_{i}$ and any generalized U(1) connection $\tilde{W}$ that is flat except at the punctures and has singularities of standard form at the punctures with respect to the chosen coordinate system on $U$,

$$
W=\tilde{W}+\sum_{i=1}^{n-1}\left(x_{i} X_{i}+y_{i} Y_{i}\right)
$$

defines a generalized $\mathrm{U}(1)$ connection that is flat except at the punctures and has singularities of standard form.

Note that property (d) implies the 1-forms $X_{i}, Y_{i}$ are closed.

One can show that 1-forms with properties (a)-(d) exist using deRham cohomology, but a more explicit construction is perhaps more 
illuminating. We actually define $X_{i}$ and $Y_{i}$ on regions slightly larger than $S-\mathcal{P}$. Define $X_{i}$ on all of $S$ by

$$
X_{i}=d f_{i}
$$

where $f_{i}$ is a smooth real-valued function on $S$ with $f_{i}\left(p_{j}\right)=1$ for $j \neq i$ and $f_{i}=0$ in an open disc containing $p_{i}$ and the loop $\eta_{i}$. To define $Y_{i}$, first set

$$
Y_{i}=\frac{1}{2 \pi} \frac{(x-i) d y-y d x}{(x-i)^{2}+y^{2}}
$$

in an open disc containing $p_{i}$ and the loop $\eta_{i}$, and

$$
Y_{i}=-\frac{1}{2 \pi} \frac{(x-n) d y-y d x}{(x-n)^{2}+y^{2}}
$$

in an open disc containing $p_{n}$. Then extend $Y_{i}$ smoothly to a closed 1 -form on all of $S-\left\{p_{i}, p_{n}\right\}$. One can check that with these definitions, $X_{i}$ and $Y_{i}$ have properties (a)-(d).

One can show that by applying a suitable gauge transformation in $\mathcal{G}^{\mathcal{P}}$, any connection of the form given by equation (22) can be brought into the special form given by equation (23). Thus, given any point $[W] \in \mathcal{X}^{\mathcal{P}}$, we can choose a representative $W \in \mathcal{X}^{\mathcal{P}}$ with the form given in equation (23). Define

$$
g_{i}=W\left(\gamma_{i}\right), \quad h_{i}=W\left(\eta_{i}\right) .
$$

Using the chosen trivialization of $Q$ over the open $\operatorname{disc} U$, we may think of the holonomies $g_{i}$ and $h_{i}$ as elements of $\mathrm{U}(1)$. Gauge transformations in $\mathcal{G}^{\mathcal{P}}$ leave these holonomies unchanged, because such gauge transformations equal the identity at the punctures. With more work, one can also show that all bundle automorphisms in $\mathcal{G}^{\mathcal{P}} \rtimes \mathcal{D}^{\mathcal{P}}$ leave these holonomies unchanged. (This is the reason for our somewhat complicated definition of $\mathcal{D}^{\mathcal{P}}$.) It follows that these holonomies depend only on the equivalence class $[W]$, so they define functions

$$
g_{i}, h_{i}: \mathcal{X}^{\mathcal{P}} \rightarrow \mathrm{U}(1) \text {. }
$$

Taking all these functions together we obtain a map

$$
\Phi: \mathcal{X}^{\mathcal{P}} \rightarrow(\mathrm{U}(1) \times \mathrm{U}(1))^{n-1}
$$

with

$$
\Phi([W])=\left(g_{1}, h_{1}, \ldots, g_{n-1}, h_{n-1}\right)
$$


To show that $\mathcal{X}^{\mathcal{P}}$ is a $2(n-1)$-dimensional torus, we just need to show that $\Phi$ is a diffeomorphism. It is clearly smooth. To see that $\Phi$ is onto, note that the generalized connection $W$ given in equation (23) has

$$
g_{i}(W)=e^{i x_{i}}, \quad h_{i}(W)=e^{i y_{i}} .
$$

To see that $\Phi$ is one-to-one, use deRham cohomology.

Since the map $\Phi$ constructed in the above proof is a diffeomorphism, we can use the functions $x_{i}$ and $y_{i}$ as 'coordinates' on $\mathcal{X}^{\mathcal{P}}$. Of course, these coordinates are only defined modulo $2 \pi$. More precisely, there is a covering

$$
\begin{aligned}
\mathbb{R}^{2(n-1)} & \rightarrow \mathrm{U}(1)^{2(n-1)} \cong \mathcal{X}^{\mathcal{P}} \\
\left(x_{i}, y_{i}\right) & \mapsto\left(e^{i x_{i}}, e^{i y_{i}}\right),
\end{aligned}
$$

so we can identify $\mathcal{X}^{\mathcal{P}}$ with $\mathbb{R}^{2(n-1)}$ modulo the lattice

$$
\Lambda=(2 \pi \mathbb{Z})^{2(n-1)} \text {. }
$$

In terms of these coordinates, it turns out that the physically relevant symplectic structure on $\mathcal{X}^{\mathcal{P}}$ is given by

$$
\omega=\frac{k}{2 \pi} \sum_{i=1}^{n-1} d x_{i} \wedge d y_{i}
$$

To see this, note that the physically relevant symplectic structure on $\mathcal{X}^{\mathcal{P}}$ is given by

$$
\omega\left([\delta W],\left[\delta W^{\prime}\right]\right)=\frac{k}{2 \pi} \int_{S^{2}} \delta W \wedge \delta W^{\prime}
$$

where $[\delta W],\left[\delta W^{\prime}\right]$ are tangent vectors to $\mathcal{X}^{\mathcal{P}}$ corresponding to tangent vectors $\delta W, \delta W^{\prime}$ at some point of $\mathcal{A}^{\mathcal{P}}$. This formula comes from the surface term in equation (7), which was derived in the context of smooth connections. However, it makes sense even for generalized connections, as long as $\delta W$ and $\delta W^{\prime}$ are linear combinations of the 1-forms $X_{i}$ and $Y_{i}$, which we may assume without loss of generality. Using property (c) of the 1-forms $X_{i}$ and $Y_{i}$ (as described in the proof of the above theorem), we may write

$$
\delta W=\sum_{i=1}^{n-1}\left(\delta x_{i} X_{i}+\delta y_{i} Y_{i}\right), \quad \delta W^{\prime}=\sum_{i=1}^{n-1}\left(\delta x_{i}^{\prime} X_{i}+\delta y_{i}^{\prime} Y_{i}\right)
$$


This implies that

$$
\omega\left(\delta W, \delta W^{\prime}\right)=\frac{k}{2 \pi} \int_{S^{2}} \operatorname{Tr}\left(\delta W \wedge \delta W^{\prime}\right)=\frac{k}{2 \pi} \sum_{i=1}^{n-1}\left(\delta x_{i} \delta y_{i}^{\prime}-\delta y_{i} \delta x_{i}^{\prime}\right)
$$

proving equation (24).

To summarize, in this section we gave a precise definition of generalized connections which are flat everywhere except at the finite set of points $\mathcal{P}$, proved that the resulting phase space $\mathcal{X}^{\mathcal{P}}$ is diffeomorphic to a $2(n-1)$-dimensional torus, and showed that the Chern-Simons surface term in the symplectic structure (7) of the classical theory is well-defined on $\mathcal{X}^{\mathcal{P}}$ in spite of the fact that the curvature of the connections now under consideration is distributional.

Note that in the proof of Theorem 1, we needed to introduce some extra structure on the surface $S$ to construct the map $\Phi$ that identifies $\mathcal{X}^{\mathcal{P}}$ with the torus $(\mathrm{U}(1) \times \mathrm{U}(1))^{n-1}$. In the next section, our identification of $\mathcal{X}^{\mathcal{P}}$ with this torus plays a crucial role in the geometric quantization. By definition, the map $\Phi$ is invariant under the action of diffeomorphisms in $\mathcal{D}^{\mathcal{P}}$. However, $\Phi$ is not invariant under all diffeomorphisms of $S$-not even those that fix each puncture in $\mathcal{P}$ - because the extra structure needed to define this map transforms nontrivially under such diffeomorphisms. Thus, in order to study the diffeomorphism constraint in Section 5.2.2, it is important to know precisely what extra structure was needed to define the map $\Phi$.

First, of course, we needed to fix an ordering $p_{1}, \ldots, p_{n}$ of the punctures in $\mathcal{P}$. Then we needed to fix a ray in the tangent space of each puncture and a trivialization of $Q$ at each puncture. Then we needed to choose real-analytic paths $\gamma_{i}$ going from $p_{i}$ to $p_{n}$, whose tangent vectors at both endpoints lie in the chosen rays. We required that these paths look as in Figure 4 with respect to some analytic coordinate system. However, we can continuously deform the paths $\gamma_{i}$ without changing the holonomies along these paths, as long as we keep their tangent vectors at at the endpoints fixed throughout the deformation, and make sure their interiors stay in $S-\mathcal{P}$ throughout the deformation. We thus need only a certain equivalence class of paths $\gamma_{i}$. We shall use the notation $\mathcal{S}$ to stand for a choice of the ordering, rays and equivalence classes $\gamma_{i}$. One can check that $\Phi$ is uniquely defined given $\mathcal{P}$ and $\mathcal{S}$. 


\subsubsection{Geometric quantization of $\mathcal{X}^{\mathcal{P}}$}

To geometrically quantize the phase space $\mathcal{X}^{\mathcal{P}}$, we first give it a Kähler structure having the symplectic structure $\omega$ as its imaginary part. Then we find a holomorphic line bundle $L$ over $\mathcal{X}^{\mathcal{P}}$ equipped with a connection $\nabla$ whose curvature is $i \omega$. This 'prequantum line bundle' only exists when the level $k$ is an integer. Then we construct the Hilbert space of holomorphic sections of $L$. We call this Hilbert space $\mathcal{H}_{S}^{\mathcal{P}}$, since states in this space describe quantum geometries of the horizon $S$ which are flat except at the punctures in the set $\mathcal{P}$. Finally, we construct the surface Hilbert space $\mathcal{H}_{S}$ by putting all these spaces $\mathcal{H}_{S}^{\mathcal{P}}$ together in a suitable way.

To start, first note that we can introduce complex local coordinates $z_{i}=x_{i}+i y_{i}$ on the space $\mathcal{X}^{\mathcal{P}}$. In other words, we can use the standard isomorphism $\mathbb{R}^{2(n-1)} \cong \mathbb{C}^{n-1}$ to make the identification

$$
\mathcal{X}^{\mathcal{P}} \cong \mathbb{C}^{n-1} / \Lambda
$$

so that $\mathcal{X}^{\mathcal{P}}$ becomes a complex manifold. In fact, it becomes a Kähler manifold with a Kähler structure $g$ coming from $\frac{k}{2 \pi}$ times the usual Kähler structure on $\mathbb{C}^{n-1}$. This Kähler structure is compatible with the symplectic structure given in equation (24), in the sense that $\operatorname{Im} g=\omega$.

Next we construct a holomorphic complex line bundle $L$ over $\mathcal{X}^{\mathcal{P}}$ with a connection $\nabla$ whose curvature is $i \omega$. Here we use some ideas described in Mumford's book on theta functions [31]. We first introduce $\nabla$ as a connection on the trivial complex line bundle over $\mathbb{C}^{n-1}$, given by:

$$
\nabla_{x_{i}}=\partial_{x_{i}}, \quad \nabla_{y_{i}}=\partial_{y_{i}}+\frac{i k}{2 \pi} z_{i}
$$

Note that

$$
\left[\nabla_{x_{i}}, \nabla_{x_{j}}\right]=0, \quad\left[\nabla_{y_{i}}, \nabla_{y_{j}}\right]=0, \quad\left[\nabla_{x_{i}}, \nabla_{y_{j}}\right]=\frac{i k}{2 \pi} \delta_{i j}
$$

so the curvature of this connection is $\frac{i k}{2 \pi}$ times the standard symplectic structure on $\mathbb{C}^{n-1}$. Using this connection, we define parallel translation operators

$$
U_{i}(t)=\exp \left(t \nabla_{x_{i}}\right), \quad V_{i}(t)=\exp \left(t \nabla_{y_{i}}\right)
$$


for all $t \in \mathbb{R}$. More explicitly, we have:

$$
\begin{aligned}
& \left(U_{i}(t) \psi\right)\left(z_{1}, \ldots, z_{n-1}\right)=\psi\left(z_{1}, \ldots, z_{i}+t, \ldots, z_{n-1}\right) \\
& \left(V_{i}(t) \psi\right)\left(z_{1}, \ldots, z_{n-1}\right)=e^{\frac{k}{2 \pi}\left(i t z_{i}-\frac{1}{2} t^{2}\right)} \psi\left(z_{1}, \ldots, z_{i}+i t, \ldots, z_{n-1}\right) .
\end{aligned}
$$

These parallel translation operators are clearly 1-parameter groups:

$$
U_{i}(s) U_{i}(t)=U_{i}(s+t), \quad V_{i}(s) V_{i}(t)=V_{i}(s+t),
$$

and the above commutation relations imply

$$
\begin{gathered}
U_{i}(s) U_{j}(t)=U_{j}(t) U_{i}(s), \quad V_{i}(s) V_{j}(t)=V_{j}(t) V_{i}(s) \\
U_{i}(s) V_{j}(t)=e^{\frac{i k}{2 \pi} s t} V_{j}(t) U_{i}(s) .
\end{gathered}
$$

The parallel translation operators give a representation of the lattice $\Lambda$ on the space of holomorphic functions on $\mathbb{C}^{n-1}$. Given $w=u+i v$ with $u, v \in \mathbb{R}^{n-1}$, let

$$
R(w)=U_{1}\left(u_{1}\right) \cdots U_{n-1}\left(u_{n-1}\right) V_{1}\left(v_{1}\right) \cdots V_{n-1}\left(v_{n-1}\right) .
$$

Using the explicit formulas for the parallel translation operators it is clear that $R(w)$ maps holomorphic functions to holomorphic functions. We also have

$$
R\left(w+w^{\prime}\right)=e^{-\frac{i k}{2 \pi} u^{\prime} \cdot v} R(w) R\left(w^{\prime}\right)
$$

when $w^{\prime}=u^{\prime}+i v^{\prime}$ with $u^{\prime}, v^{\prime} \in \mathbb{R}^{n-1}$. Thus $R$ is a projective representation of $\mathbb{C}^{n-1}$ on the space of holomorphic functions. If $w$ and $w^{\prime}$ lie in the lattice $\Lambda$ then the above formula reduces to

$$
R\left(w+w^{\prime}\right)=R(w) R\left(w^{\prime}\right) .
$$

Thus $R$ restricts to an honest representation of $\Lambda$.

Now, the representation $R$ comes from an action of $\Lambda$ on the trivial line bundle over $\mathbb{C}^{n-1}$. The quotient of this bundle by this group action is a line bundle over $\mathbb{C}^{n-1} / \Lambda=\mathcal{X}^{\mathcal{P}}$, which we denote by $L$. The connection $\nabla$ gives rise to a connection on $L$ which we also call $\nabla$. The curvature of this connection is $i \omega$, as desired.

Next let us describe the space of holomorphic sections of $L$. A holomorphic section of $L$ is the same as a holomorphic function on $\mathbb{C}^{n-1}$ that is invariant under the operators $R(w)$ for all $w \in \Lambda$. Such functions 
are called 'theta functions' [31, 32]. We can obtain theta functions by the technique of group averaging. To make our job easier, suppose we start with a holomorphic function $f$ on $\mathbb{C}^{n-1}$ that is invariant under $R(u)$ for real lattice vectors $u$, that is, for $u \in(2 \pi \mathbb{Z})^{n-1}$. Then we can try to average $f$ with respect to the imaginary lattice directions, forming the function

$$
\psi=\sum_{v \in(2 \pi \mathbb{Z})^{n-1}} R(i v) f
$$

If the sum converges uniformly on compact subsets of $\mathbb{C}^{n-1}$, it defines a theta function.

It is easy to find holomorphic functions that are invariant under $R(u)$ for real lattice vectors $u$, since this condition simply amounts to periodicity in the real lattice directions. A basis of such functions is given by

$$
f_{a}(z)=\exp (i a \cdot z)
$$

where $a \in \mathbb{Z}^{n-1}$. If we apply the group averaging technique to such a function $f_{a}$, we obtain a theta function

$$
\psi_{a}(z)=\sum_{v \in(2 \pi \mathbb{Z})^{n-1}} e^{\frac{k}{2 \pi}\left(i v \cdot z-\frac{1}{2} v \cdot v\right)} e^{i a \cdot(z+i v)}
$$

since the sum indeed converges uniformly on compact subsets. In fact the functions $\psi_{a}$ form a basis of theta functions as we let $a$ range over vectors with $a_{i} \in\{1, \ldots, k\}$ for all $i$. The reason for this restriction is that $\psi_{a}$ changes by only a scalar factor if we add to $a$ a vector lying in $(k \mathbb{Z})^{n-1}$. For a full proof that the functions $\psi_{a}$ form a basis of theta functions, see Mumford's book [31].

Let $\mathcal{H}_{S}^{\mathcal{P}}$ denote the space of holomorphic sections of $L$, or equivalently, the space of theta functions. By the above remarks, the dimension of this space is $k^{n-1}$. We make this space into a Hilbert space as follows: given theta functions $f$ and $g$, and defining $z=x+i y$ for $x, y \in \mathbb{R}^{n-1}$, we define their inner product by

$$
\langle f, g\rangle=\int_{[0,2 \pi]^{2(n-1)}} e^{-\frac{k}{2 \pi} y \cdot y} \bar{f}(z) g(z) d^{n-1} x d^{n-1} y .
$$

This integral is easily seen to converge when $f, g$ lie in the above basis of theta functions, so it converges for all theta functions. 
As we have just seen, the Hilbert space $\mathcal{H}_{S}^{\mathcal{P}}$ has a basis given by the states $\psi_{a}$ where $a=\left(a_{1}, \ldots, a_{n-1}\right)$ with $a_{i} \in\{1, \ldots, k\}$ for all $i$. In fact, it is more convenient to regard the $a_{i}$ as elements of $\mathbb{Z}_{k}$. Also, to avoid treating the point $p_{n}$ differently from the rest, we shall change our notation slightly and treat $a$ as an $n$-tuple $\left(a_{1}, \ldots, a_{n}\right) \in \mathbb{Z}_{k}^{n}$, where we define $a_{n}$ by requiring that

$$
a_{1}+\cdots+a_{n}=0 .
$$

Taking advantage of this change of viewpoint, and adding a superscript ' $\mathcal{P}$ ' to denote the dependence on the set of punctures, it follows that $\mathcal{H}_{S}^{\mathcal{P}}$ has a basis $\psi_{\mathcal{P}, a}$ where $a$ ranges over all elements of $\mathbb{Z}_{k}^{n}$ satisfying the above equation. Note that the dimension of $\mathcal{H}_{S}^{\mathcal{P}}$ is $k^{n-1}$, just as one would predict using the Bohr-Sommerfeld rule of 'one quantum state per unit volume of phase space', where phase space volume is measured in units of $h^{n-1}$.

We conclude by defining the 'surface Hilbert space' $\mathcal{H}_{S}$, which is built from all the Hilbert spaces $\mathcal{H}_{S}^{\mathcal{P}}$ as the set of punctures $\mathcal{P}$ is allowed to vary. We could do this using a direct limit construction, but due to subtleties involving the extra structure needed to quantize the phase spaces $\mathcal{X}^{\mathcal{P}}$, as described at the end of Section 4.3.1, it is more efficient to use the following construction. Let $\mathcal{H}_{S}^{\mathcal{P}, a}$ be the one-dimensional subspace of $\mathcal{H}_{S}^{\mathcal{P}}$ spanned by the state $\psi_{\mathcal{P}, a}$, and let

$$
\mathcal{H}_{S}=\bigoplus_{\mathcal{P}, a} \mathcal{H}_{S}^{\mathcal{P}, a}
$$

where $\mathcal{P}, a$ ranges over all finite subsets of $S$ labelled with nonzero elements $a_{i} \in \mathbb{Z}_{k}$ that sum to zero. The reason we demand that the $a_{i}$ be nonzero is as follows. As we shall see in Section VA2, when $a_{i}=0$ the vector $\psi_{\mathcal{P}, a}$ corresponds to a quantum state in which the $\mathrm{U}(1)$ connection is flat at the point $p_{i}$. Such states already appear in the Hilbert space $\mathcal{H}_{S}^{\mathcal{P}-\left\{p_{i}\right\}}$, so to avoid 'double counting' these states, we exclude labellings $a$ where any $a_{i}$ equals zero. (A direct limit construction would automatically avoid this 'double counting'.)

It follows immediately that the surface Hilbert space has a basis of states $\psi_{\mathcal{P}, a}$ corresponding to all ways of choosing finitely many distinct points $p_{1}, \ldots, p_{n}$ of $S$ and labelling these points with nonzero numbers $a_{1}, \ldots, a_{n} \in \mathbb{Z}_{k}$ satisfying

$$
a_{1}+\cdots+a_{n}=0
$$


Thus, we have proved the assertions about the surface Hilbert space $\mathcal{H}_{S}$ made in Section 3. In Section 5.1.2 we give a geometrical interpretation of the basis states $\psi_{\mathcal{P}, a}$ and of the above condition on the numbers $a_{i}$. These states will enable us to impose the quantum boundary conditions (13) explicitly.

\section{$5 \quad$ Physical States}

\subsection{Quantum Boundary Condition}

Having constructed the volume and surface Hilbert spaces, we now wish to impose the quantum boundary condition, in order to pick out the kinematical Hilbert space $\mathcal{H}$ as a subspace of $\mathcal{H}_{V} \otimes \mathcal{H}_{S}$. The naive version of this condition, equation (11), simply states that kinematical states must be invariant under all $\mathrm{U}(1)$ gauge transformations on the horizon surface $S$. This suggests that we study the action of such gauge transformations on the volume and surface Hilbert spaces.

In Section 5.1.1 we study the action of U(1) gauge transformations on the volume Hilbert space. In Section 5.1.2 we carry out a similar study for the surface Hilbert space, but we encounter an important subtlety: there is no operator $\hat{F}$ generating the action of $\mathrm{U}(1)$ gauge transformations on $\mathcal{H}_{S}$. Instead, there is only a unitary operator corresponding to the exponential $\exp (i \hat{F})$. Mathematically speaking, what this means is that only gauge transformations taking values in a certain discrete subgroup of $\mathrm{U}(1)$ act as unitary operators on $\mathcal{H}_{S}$. This subgroup turns out to be $\mathbb{Z}_{k} \subset \mathrm{U}(1)$, the subgroup consisting of all $k$ th roots of unity, where $k$ is the level defined in equation (15).

For this reason, we need to impose the quantum boundary condition in its exponentiated form, equation (12), rather than the naive form given in equation (11). In its exponentiated form, the quantum boundary condition states that kinematical states must be invariant under all $\mathbb{Z}_{k}$-valued gauge transformations on $S$. Starting from this fact, in Section (5.1.3) we explicitly describe the kinematical Hilbert space $\mathcal{H}$ as a subspace of $H_{V} \otimes \mathcal{H}_{S}$. 


\subsubsection{Action of gauge transformations on $\mathcal{H}_{V}$}

First we describe operators on the volume Hilbert space that implement $\mathrm{U}(1)$ gauge transformations at the horizon. Most of our work is already done, because in Section 4.2 we described how arbitrary $\mathrm{SU}(2)$ gauge transformations at the horizon act on the volume Hilbert space. Here, however, we want a more explicit description of how the subgroup consisting of $U(1)$ gauge transformations acts on the volume Hilbert space, so we can implement the quantum boundary condition.

Recall now that $\mathcal{G}_{S}$ stands for the group of all (not necessarily continuous) gauge transformations of the bundle $\left.P\right|_{S}$. This has a subgroup $\mathcal{G}_{Q}$ consisting of those gauge transformations that fix the radial internal vector field $r: S \rightarrow \mathfrak{s u}(2)$. Alternatively, we can think of $\mathcal{G}_{Q}$ as consisting of all gauge transformations of the $\mathrm{U}(1)$ bundle $Q$, which abstractly is just the spin bundle of the sphere $S$.

Since $\mathcal{G}_{S}$ has a unitary representation on $\mathcal{H}_{V}$, so does the subgroup $\mathcal{G}_{Q}$. In particular, if we pick any point $p \in S$, we obtain a unitary representation of $\mathrm{U}(1)$ on $\mathcal{H}_{V}$ by considering gauge transformations in $\mathcal{G}_{Q}$ that are the identity at every point except $p$. In the notation of Section 4.2 , the self-adjoint infinitesimal generator of $U(1)$ action is the operator

$$
J(p) \cdot r=J^{i}(p) r_{i}
$$

Let $\mathcal{P}=\left\{p_{1}, \ldots, p_{n}\right\}$ be any finite subset of $S$, and let $m$ stand for a way of labelling each point $p_{i}$ with a nonzero half-integer $m_{i}$. Then there is a subspace $\mathcal{H}_{V}^{\mathcal{P}, m}$ of the volume Hilbert space consisting of all vectors $\psi$ such that

$$
\left(J\left(p_{i}\right) \cdot r\right) \psi=m_{i} \psi
$$

for each point $p_{i}$ and

$$
(J(p) \cdot r) \psi=0
$$

for all points $p$ other than the points $p_{i}$. Clearly $\mathcal{H}_{V}^{\mathcal{P}, m}$ is a subspace of $\mathcal{H}_{V}^{\mathcal{P}}$, since gauge transformations at any point $p \in S$ act trivially on states corresponding to spin networks that do not have $p$ as one of their ends. Moreover, we have a direct sum decomposition

$$
\mathcal{H}_{V}=\bigoplus_{\mathcal{P}, m} \mathcal{H}_{V}^{\mathcal{P}, m}
$$


where $\mathcal{P}, m$ ranges over all finite sets of points in $S$ labelled by nonzero half-integers. This is analogous to the decomposition of the surface Hilbert space into subspaces $\mathcal{H}_{S}^{\mathcal{P}, a}$ given in equation (32). The reader may also wish to compare this direct sum decomposition of the volume Hilbert space with the previous decomposition, given in equation (19), based on eigenspaces of the operator $J(p) \cdot J(p)$ which features in the expression of quantum area.

The present decomposition of the volume Hilbert space diagonalizes the action of $\mathcal{G}_{Q}$, in the following sense. Suppose $g \in \mathcal{G}_{Q}$ and $\psi \in \mathcal{H}_{V}^{\mathcal{P}, m}$. Then for each point $p_{i} \in \mathcal{P}$ we can think of $g\left(p_{i}\right)$ as an element of $\mathrm{U}(1)$, and

$$
g \psi=g\left(p_{1}\right)^{2 m_{1}} \cdots g\left(p_{n}\right)^{2 m_{n}} \psi .
$$

The factors of 2 here come from the usual convention of using a halfinteger $m$ to label the eigenvalues of a specific component of angular momentum.

\subsubsection{Action of gauge transformations on $\mathcal{H}_{S}$}

Next we describe operators on the surface Hilbert space that implement gauge transformations at the punctures. Classically the group of allowed gauge transformations at each puncture is $\mathrm{U}(1)$. However, not all these gauge transformations can be implemented as operators on the surface Hilbert space, but only those lying in the discrete subgroup $\mathbb{Z}_{k} \subset \mathrm{U}(1)$, where $k$ is the level defined in equation (15). Thus in a sense the group of gauge transformations must itself be 'quantized' as part of the quantization procedure.

In fact, the 'quantization of the gauge group' we see here is a simple example of the relation between Chern-Simons theory and quantum groups [33]. Recall that the surface Hilbert space is obtained by quantizing the phase space for U(1) Chern-Simons theory on a sphere with a fixed set of punctures, and then allowing the set of punctures to vary. Since Chern-Simons theory can also be described using quantum groups, one expects an alternative description of the surface Hilbert space in terms of representations of 'quantum U(1)'. This quantum group is essentially just the group $\mathbb{Z}_{k}$. More precisely, it is the usual Hopf algebra associated to $\mathbb{Z}_{k}$, but equipped with a modified quasitriangular structure [34]. 
However, we do not need the machinery of quantum groups in what follows, since we can instead use well-known facts about about theta functions. As we saw in Section 4.3.2, when there are $n$ punctures the classical phase space $\mathcal{X}^{\mathcal{P}}$ is a $2(n-1)$-dimensional torus, and quantum states in $\mathcal{H}_{S}^{\mathcal{P}}$ are holomorphic sections of a line bundle $L$ over this torus. For each puncture we get an action of $\mathrm{U}(1)$ as symplectic transformations of the torus. However, not all elements of $U(i)$ lift to holomorphic transformations of the line bundle, but only those lying in the subgroup $\mathbb{Z}_{k}$, so only these act as unitary operators on $\mathcal{H}_{S}^{\mathcal{P}}$. This is a standard but nontrivial result in the theory of theta functions [35], so we shall not prove it here. However, we will give an explicit construction of the lift when it exists. It should explain why the subgroup $\mathbb{Z}_{k}$ plays a special role.

As noted in Section 3, this 'quantization of the gauge group' turns out to have very striking implications for the quantum geometry of the horizon. It means that the holonomy of the $\mathrm{U}(1)$ connection around each puncture $p_{i}$ can only take on a discrete spectrum of values: namely, those of the form $\exp \left(2 \pi i a_{i} / k\right)$ where $a_{i} \in \mathbb{Z}_{k}$. Heuristically, this means the horizon is flat except at the punctures, where there are conical singularities with quantized angle deficits.

To begin, let $\mathcal{G}_{Q}$ be the group of all gauge transformations of the bundle $Q$. This group acts on $\mathcal{A}^{\mathcal{P}}$, and this action descends to an action on the phase space $\mathcal{X}^{\mathcal{P}}$, where we have quotiented by the action of the subgroup $\mathcal{G}^{\mathcal{P}} \rtimes \mathcal{D}^{\mathcal{P}}$. However, the action of any gauge transformation $g$ on $\mathcal{X}^{\mathcal{P}}$ depends only on its values $g\left(p_{i}\right)$, so we obtain an action of $\mathrm{U}(1)^{n}$ on $\mathcal{X}^{\mathcal{P}}$. Another way to see this is to note that

$$
\mathcal{G}_{Q} / \mathcal{G}^{\mathcal{P}}=\mathrm{U}(1)^{n}
$$

The action of a gauge transformation at the point $p_{n}$ can be reexpressed in terms of gauge transformations at the other points $p_{i}$, because constant gauge transformations act trivially on $\mathcal{X}^{\mathcal{P}}$. Thus we begin by only considering gauge transformations that are the identity except at the first $n-1$ points. We denote the action of these gauge transformations on the phase space as

$$
T: \mathrm{U}(1)^{n-1} \times \mathcal{X}^{\mathcal{P}} \rightarrow \mathcal{X}^{\mathcal{P}} .
$$

In terms of the holonomies $g_{i}, h_{i}$, we have the following explicit formula 
for $T$ :

$$
\begin{aligned}
& T\left(e^{i \theta_{1}}, \ldots, e^{i \theta_{n-1}}\right)\left(g_{1}, h_{1}, \ldots, g_{n-1}, h_{n-1}\right) \\
& =\left(e^{-i \theta_{1}} g_{1}, h_{1}, \ldots, e^{-i \theta_{n-1}} g_{n-1}, h_{n-1}\right) .
\end{aligned}
$$

In other words, $T$ is just the action of $U(1)^{n-1}$ by translations on $\mathcal{X}^{\mathcal{P}} \cong$ $(\mathrm{U}(1) \times U(1))^{n-1}$.

Given an element $g$ of $\mathrm{U}(1)^{n-1}$, we can now ask if $T(g)$ lifts to a holomorphic transformation of the line bundle $L$. As mentioned above, this is possible if and only if $g$ lies in the subgroup $\mathbb{Z}_{k}^{n-1}$. In this case, there is a natural way to choose a lift that defines a unitary operator on the Hilbert space $\mathcal{H}_{S}^{\mathcal{P}}$. To see this, suppose that $g \in \mathrm{U}(1)^{n-1}$ and choose an element $w \in \mathbb{R}^{n-1}$ that is sent to $g$ by the covering map

$$
\mathbb{R}^{n-1} \rightarrow(\mathbb{R} / 2 \pi \mathbb{Z})^{n-1}=\mathrm{U}(1)^{n-1} .
$$

Associated to $w$ there is an operator $R(w)$ on the space of holomorphic functions on $\mathbb{C}^{n-1}$, given by equation (28). This operator preserves the space of theta functions if it commutes with $R\left(w^{\prime}\right)$ for all $w^{\prime} \in \Lambda$. Using equation (29), one can check that this holds precisely when $w$ lies in the lattice $\left(\frac{2 \pi}{k} \mathbb{Z}\right)^{n-1}$, or equivalently, when $g$ lies in $\mathbb{Z}_{k}^{n-1}$. Because $R(w)$ is defined in terms of parallel translation with respect to the connection $\nabla$ and it preserves the space of theta functions, it really does come from a lift of $T(g)$ to a holomorphic map on bundle $L$, as desired.

As a unitary operator on the Hilbert space $\mathcal{H}_{S}^{\mathcal{P}}$, the operator $R(w)$ is independent of the choice of $w$ mapping to $g \in \mathbb{Z}_{k}^{n-1}$. We shall thus write this operator simply as $R(g)$. Using equations (28) and (31), one can check that $R$ is a unitary representation of $\mathbb{Z}_{k}^{n-1}$ on $\mathcal{H}_{S}^{\mathcal{P}}$. Moreover, at this point it is easy to go back and consider the action of gauge transformations at the point $p_{n}$ as well as the points $p_{1}, \ldots, p_{n-1}$. These give an action of $\mathrm{U}(1)^{n}$ on $\mathcal{X}^{\mathcal{P}}$, which we again call $T$. This is given explicitly as follows:

$$
\begin{aligned}
& T\left(e^{i \theta_{1}}, \ldots, e^{i \theta_{n}}\right)\left(g_{1}, h_{1}, \ldots, g_{n-1}, h_{n-1}\right) \\
& =\left(e^{i\left(\theta_{n}-\theta_{1}\right)} g_{1}, h_{1}, \ldots, e^{i\left(\theta_{n}-\theta_{n-1}\right)} g_{n-1}, h_{n-1}\right) .
\end{aligned}
$$

By our previous results, $T(g)$ only lifts to a holomorphic map of the line bundle $L$ when $g \in \mathbb{Z}_{k}^{n}$. This gives us a unitary representation of $\mathbb{Z}_{k}^{n}$ on $\mathcal{H}_{S}^{\mathcal{P}}$, which we again call $R$. We can think of $R$ as describing the 
action of the 'quantized gauge group' $\mathbb{Z}_{k}^{n}$ on geometries of the horizon for which the $\mathrm{U}(1)$ connection is flat except at the points $p_{i}$.

To describe $R$ more explicitly, we can use the basis $\psi_{a}$ of $\mathcal{H}_{S}^{\mathcal{P}}$ described in Section 4.3.2. Recall that here $a=\left(a_{1}, \ldots, a_{n}\right)$ is any $n$-tuple of integers modulo $k$ that sum to zero. Suppose $\left(g_{1}, \ldots, g_{n}\right) \in \mathbb{Z}_{k}^{n}$. Then using the formulas for the states $\psi_{a}$ and the representation $R$, one can show that

$$
R\left(g_{1}, \ldots, g_{n}\right) \psi_{a}=g_{1}^{a_{1}} \cdots g_{n}^{a_{n}} \psi_{a}
$$

where we think of each $g_{i}$ as an element of U(1) using the inclusion $\mathbb{Z}_{k} \subset \mathrm{U}(1)$. Note that this formula makes sense even though the integers $a_{i}$ are only defined modulo $k$, thanks to the fact that the phases $g_{i}$ are $k$ th roots of unity.

How can we understand the meaning of equation (36)? Naively, we would expect the quantity $\frac{2 \pi}{k} F$ to generate $\mathrm{U}(1)$ gauge transformations on the horizon, where $F$ is the curvature of the $\mathrm{U}(1)$ connection $W$, thanks to the surface term in the symplectic structure in equation 25 . This would suggest that the curvature at a puncture is the infinitesimal generator of gauge transformations at that puncture. But the curvature at a puncture is not a well-defined function on $\mathcal{X}^{\mathcal{P}}$, since this phase space is defined in terms of generalized connections. Instead, as a kind of substitute, we have a function $h_{i}$ on $\mathcal{X}^{\mathcal{P}}$ measuring the holonomy around the loop $\eta_{i}$ enclosing the $i$ th puncture, as shown in Figure 4. Since this holonomy is essentially the exponential of the curvature concentrated at the $i$ th puncture, we expect the corresponding operator in the quantized theory to be the exponential of the generator of gauge transformations at $p_{i}$ - i.e., a unitary operator corresponding to a finite gauge transformation at $p_{i}$. The obvious candidate for this operator is

$$
\hat{h}_{i}=R\left(1, \cdots, 1, e^{\frac{2 \pi i}{k}}, 1, \ldots, 1\right),
$$

all the entries being 1 except the $i$ th.

By equation (36), the states $\psi_{a}$ satisfy

$$
\hat{h}_{i} \psi_{a}=e^{\frac{2 \pi i a_{i}}{k}} \psi_{a}
$$

The above heuristic argument thus suggests that $\psi_{a}$ represents a quantum geometry for the horizon in which the connection $W$ is flat except at the punctures and has holonomy $\exp \left(2 \pi i a_{i} / k\right)$ around the $i$ th puncture. The condition that $a_{1}+\cdots+a_{n}=0$ then says that the product 
of all these holonomies is 1 . This is exactly what one would expect, because the product of all these holonomies equals the holonomy around a loop winding once around all the punctures, and this sort of loop is contractible in $S-\mathcal{P}$.

In the state $\psi_{a}$ we can visualize the horizon as having conical singularities with specified angle deficits at the punctures, as shown in Figure 3. Of course, $W$ is a connection on the spin bundle of the horizon; in terms of the $\mathrm{SO}(2)$ 'Levi-Civita' connection on the tangent bundle, the angle deficit at the $i$ th puncture is $4 \pi a_{i} / k$. Since $a_{i}$ is only defined modulo $k$, this angle deficit is only defined modulo $4 \pi$. The condition $a_{1}+\cdots+a_{n}=0$ then says that the sum of these angle deficits vanishes modulo $4 \pi$. This is the quantum analogue of the classical fact that for any metric on the 2-sphere, the integral of the scalar curvature is $4 \pi$.

Our last order of business is to describe the action of gauge transformations on the surface Hilbert space $\mathcal{H}_{S}$. This is straightforward given their action on the Hilbert spaces $\mathcal{H}_{S}^{\mathcal{P}}$. To make precise the fact that only $\mathbb{Z}_{k}$-valued gauge transformations can be implemented in the quantum theory, we define $\hat{\mathcal{G}}_{Q}$, the group of 'quantized gauge transformations', to consist of all functions $g: S \rightarrow \mathbb{Z}_{k}$. We can think of this as a subgroup of the group $\mathcal{G}_{Q}$. The quantized gauge group has a unitary representation on $\mathcal{H}_{S}$, defined as follows: for any group element $g \in \hat{\mathcal{G}}_{Q}$ and vector $\psi \in \mathcal{H}_{S}^{\mathcal{P}}$ we have

$$
g \psi=R\left(g\left(p_{1}\right), \ldots, g\left(p_{n}\right)\right) \psi
$$

This implies that

$$
g \psi_{\mathcal{P}, a}=g\left(p_{1}\right)^{a_{1}} \cdots g\left(p_{n}\right)^{a_{n}} \psi_{\mathcal{P}, a}
$$

where $\psi_{\mathcal{P}, a}$ is the basis of the surface Hilbert space described at the end of Section 4.3.2. Since we defined $\mathcal{H}_{S}^{\mathcal{P}, a}$ to be the subspace of $\mathcal{H}_{S}$ spanned by the state $\psi_{\mathcal{P}, a}$, we may also say this as follows:

$$
g \psi=g\left(p_{1}\right)^{a_{1}} \cdots g\left(p_{n}\right)^{a_{n}} \psi
$$

for any $g \in \hat{\mathcal{G}}_{Q}$ and $\psi \in \mathcal{H}_{S}^{\mathcal{P}, a}$. This formula is analogous to formula (34), which describes the action of gauge transformations on the volume Hilbert space. 


\subsubsection{Implementing the quantum boundary condition}

In Section 5.1.1 we described how the group $\mathcal{G}_{Q}$ of $\mathrm{U}(1)$ gauge transformations on the horizon acts on the volume Hilbert space, while in Section 5.1.2, we described how the subgroup $\hat{\mathcal{G}}_{Q}$ consisting of $\mathbb{Z}_{k}$-valued gauge transformations acts on the surface Hilbert space. It is now a simple matter to implement the quantum boundary condition, by picking out the subspace of $\mathcal{H}_{V} \otimes \mathcal{H}_{S}$ that is invariant under the action of $\hat{\mathcal{G}}_{Q}$.

Recall from equation (33) that the volume Hilbert space is a direct sum of subspaces $\mathcal{H}_{V}^{\mathcal{P}, m}$, where $\mathcal{P}=\left\{p_{1}, \ldots, p_{n}\right\}$ ranges over all finite sets of points in $S$ and $m$ ranges over all ways of labelling these points by nonzero half-integers. By equation (34), any vector $\psi$ in $\mathcal{H}_{V}^{\mathcal{P}, m}$ transforms as follows under the action of $g \in \mathcal{G}_{Q}$ :

$$
g \psi=g\left(p_{1}\right)^{2 m_{1}} \cdots g\left(p_{n}\right)^{2 m_{n}} \psi
$$

Similarly, in equation (32) we saw that the surface Hilbert space is a direct sum of subspaces $\mathcal{H}_{S}^{\mathcal{P}, a}$, where $\mathcal{P}$ ranges over all finite sets of points in $S$ and $a$ ranges over all ways of labelling these points by nonzero elements of $\mathbb{Z}_{k}$. By equation (37), any vector $\psi$ in $\mathcal{H}_{S}^{\mathcal{P}, a}$ transforms as follows under the action of $g \in \hat{\mathcal{G}}_{Q}$ :

$$
g \psi=g\left(p_{1}\right)^{a_{1}} \cdots g\left(p_{n}\right)^{a_{n}} \psi .
$$

It follows that the subspace of $\mathcal{H}_{V} \otimes \mathcal{H}_{S}$ consisting of vectors invariant under $\hat{\mathcal{G}}_{Q}$ is

$$
\mathcal{H}^{\text {Kin }}=\bigoplus_{\mathcal{P}, m, a: 2 m=-a \bmod k} \mathcal{H}_{V}^{\mathcal{P}, m} \otimes \mathcal{H}_{S}^{\mathcal{P}, a}
$$

where $\mathcal{P}$ ranges over all finite subsets of $S, m$ ranges over all ways of labelling the points in $\mathcal{P}$ by nonzero half-integers, and $a$ ranges over all ways of labelling these points by nonzero elements of $\mathbb{Z}_{k}$ that sum to zero. We call this subspace the 'kinematical' Hilbert space.

\subsection{Quantum Einstein Equation}

As explained in Section 3, some of the classical Einstein equations contain no time derivatives and thus act as constraints on the initial data: 
the Gauss constraint, the diffeomorphism constraint, and the Hamiltonian constraint. In quantum theory, these constraints are to be incorporated, a la Dirac, as conditions on permissible physical states. In the following sections we deal with each of these three constraints in turn.

\subsubsection{Gauss constraint}

The Gauss constraint has in fact been imposed in our definition of the Hilbert space $\mathcal{H}^{\text {kin }}$ in equation (38), but let us explain exactly why this is the case. At the classical level, the Gauss constraint generates $\mathrm{SU}(2)$ gauge transformations on the pairs $(A, \Sigma)$ which reduce to $\mathrm{U}(1)$ gauge transformations preserving the internal vector field $r$ at the horizon. The group of such gauge transformations has $\mathcal{G}_{V}$ as a normal subgroup, and the quotient group is $\mathcal{G}_{Q}$. In the quantum theory, we used this decomposition to impose the Gauss constraint in two separate stages. First we restricted attention to states that are invariant under $\mathcal{G}_{V}$. Then we sought an action of $\mathcal{G}_{Q}$ on the space of such states and tried to restrict attention to states that are invariant under $\mathcal{G}_{Q}$.

The first stage of this plan was carried out in a straightforward way. The group $\mathcal{G}_{V}$ acts trivially on generalized connections on the surface, but nontrivially on the generalized connections in the volume. Thus we only needed to impose invariance under $\mathcal{G}_{V}$ for the volume degrees of freedom. In Section 4.2 we did this by defining the volume Hilbert space $\mathcal{H}_{V}$ as the subspace of $L^{2}\left(\overline{\mathcal{A}}_{V}\right)$ consisting of vectors that are invariant under $\mathcal{G}_{V}$.

The second stage of the plan required more care, and in carrying it out we encountered an important subtlety. The volume Hilbert space $\mathcal{H}_{V}$ is a direct sum of subspaces $\mathcal{H}_{V}^{\mathcal{P}, m}$ where $\mathcal{P}, m$ ranges over all finite subsets of $S$ labelled by nonzero half-integers. These subspaces have the handy property that the action of $g \in \mathcal{G}_{Q}$ on $\mathcal{H}_{V}^{\mathcal{P}, m}$ is trivial if $g$ is the identity at all points in $\mathcal{P}$. Such gauge transformations form a normal subgroup $\mathcal{G}^{\mathcal{P}} \subset \mathcal{G}_{Q}$. Taking this as our cue, we constructed the surface Hilbert space $\mathcal{H}_{S}$ as a similar direct sum of subspaces $\mathcal{H}_{S}^{\mathcal{P}, a}$, where in defining $\mathcal{H}_{S}^{\mathcal{P}}$ we imposed invariance under all gauge transformations in $\mathcal{G}^{\mathcal{P}}$. Thus, by construction, states in $\mathcal{H}_{V}^{\mathcal{P}} \otimes \mathcal{H}_{S}^{\mathcal{P}}$ are automatically invariant under the action of the subgroup $\mathcal{G}^{\mathcal{P}}$. 
To impose invariance under all of $\mathcal{G}_{Q}$, we would therefore simply need to describe the action of the quotient group $\mathcal{G}_{Q} / \mathcal{G}^{\mathcal{P}}=\mathrm{U}(1)^{n}$ on $\mathcal{H}_{V}^{\mathcal{P}} \otimes \mathcal{H}_{S}^{\mathcal{P}}$ and find the subspace of states invariant under this action. The action of $\mathrm{U}(1)^{n}$ on $\mathcal{H}_{V}^{\mathcal{P}}$ is unproblematic, and it is diagonalized by the subspaces $\mathcal{H}_{V}^{\mathcal{P}, m}$. However, it turned out that $\mathrm{U}(1)^{n}$ does not act on $\mathcal{H}_{S}^{\mathcal{P}}$; instead, thanks to the subtleties of quantum Chern-Simons theory, only the subgroup $\mathbb{Z}_{k}^{n}$ acts on this space. This action is diagonalized by the subspaces $\mathcal{H}_{S}^{\mathcal{P}, a}$. The best we can do, therefore, is to find the subspace of $\mathcal{H}_{V}^{\mathcal{P}} \otimes \mathcal{H}_{S}^{\mathcal{P}}$ consisting of vectors invariant under $\mathbb{Z}_{k}^{n}$. Using equations (34) and (37), this subspace turns out to be

$$
\bigoplus_{m, a: 2 m=-a \bmod k} \mathcal{H}_{V}^{\mathcal{P}, m} \otimes \mathcal{H}_{S}^{\mathcal{P}, a}
$$

where $m$ ranges over all ways of labelling points in $\mathcal{P}$ by half-integers and $a$ ranges over all ways of labelling these points by elements of $\mathbb{Z}_{k}$ that sum to zero.

Putting all these subspaces together, we obtain the kinematical Hilbert space

$$
\mathcal{H}^{\mathrm{Kin}}=\bigoplus_{\mathcal{P}, m, a: 2 m=-a \bmod k} \mathcal{H}_{V}^{\mathcal{P}, m} \otimes \mathcal{H}_{S}^{\mathcal{P}, a}
$$

where $\mathcal{P}$ ranges over all finite subsets of $S, m$ ranges over ways of labelling the points in $\mathcal{P}$ by nonzero half-integers, and $a$ ranges over ways of labelling them by nonzero elements of $\mathbb{Z}_{k}$ that sum to zero. This Hilbert space consists of states that are invariant under all of $\mathcal{G}_{V}$ but only the subgroup $\hat{\mathcal{G}}_{Q} \subset \mathcal{G}_{Q}$ consisting of $\mathbb{Z}_{k}$-valued gauge transformations on the horizon. Thus, while the gauge group in the classical theory is $\mathcal{G}_{V} \times \mathcal{G}_{Q}$, that in the quantum theory is reduced to $\mathcal{G}_{V} \times \hat{\mathcal{G}}_{Q}$. Explicitly, the space of states satisfying the Gauss constraint -i.e., invariant under under $\mathcal{G}_{V} \times \hat{\mathcal{G}}_{Q}$ - is the space $\mathcal{H}^{\text {Kin }}$.

\subsubsection{Diffeomorphism constraint}

Denote by $\mathcal{D}$ the group of diffeomorphisms generated by analytic vector fields on $M$ which are tangential to $S$ and tend to zero at infinity. On the classical phase space, the diffeomorphism constraint generates canonical transformations corresponding to $\mathcal{D}$. Hence states mapped to 
each other by $\mathcal{D}$ are considered physically equivalent. In the quantum theory, our task is to construct states that are invariant under the action of $\mathcal{D}$. To display the surface states 'explicitly', will use a gauge fixing procedure in which one chooses exactly one surface state in each orbit of $\mathcal{D}$. For the entropy calculation, such an explicit representation of volume states is not essential. It is easier to follow the procedure of Section 5.2.1 and impose the constraint as an operator equation.

Recall from Section 4.3.1 that in the construction of Hilbert spaces $\mathcal{H}_{S}^{\mathcal{P}, a}$ and definition of associated operators which implement $\hat{\mathcal{G}}_{Q}$ and measure deficit angles, we had to introduce an additional structure $\mathcal{S}$ on the horizon surface $S$. Thus, strictly, the surface Hilbert spaces should have been labelled $\mathcal{H}_{S}^{\mathcal{S}(\mathcal{P}), a}$. Prior to the present discussion of the diffeomorphism constraint, we omitted the explicit reference to the structure $\mathcal{S}$ for the sake of simplicity. But we can no longer do so in the present discussion, since this structure is invariant only under the subgroup $\mathcal{D}^{\mathcal{P}}$ of $\mathcal{D}$, and we now wish to investigate the action of the full group $\mathcal{D}$.

Thus, we now begin by considering the entire collection of Hilbert spaces $\mathcal{H}_{S}^{\mathcal{S}(\mathcal{P}), a}$, one for each choice of $\mathcal{S}(\mathcal{P})$. If a diffeomorphism $\phi \in \mathcal{D}$ maps $\mathcal{S}(\mathcal{P})$ to $\mathcal{S}^{\prime}\left(\mathcal{P}^{\prime}\right)$, then it provides a natural unitary mapping

$$
U_{\phi}: \mathcal{H}_{S}^{\mathcal{S}(\mathcal{P}), a} \rightarrow \mathcal{H}_{S}^{\mathcal{S}^{\prime}\left(\mathcal{P}^{\prime}\right), a}
$$

between the corresponding Hilbert spaces. The diffeomorphism constraint implies that these Hilbert spaces are physically equivalent. The 'gauge fixing procedure' we are adopting for surface states says that we should select only one copy of these physically equivalent Hilbert spaces.

It is therefore important to examine the action of $\mathcal{D}$ on the space of structures $\mathcal{S}(\mathcal{P})$ with a given number $n$ of punctures. The action is in fact transitive. The proof of this assertion is a bit technical, but the main steps can be summarized as follows. First one shows that the analogous result is true in the smooth category. For this, the main work involved is to show that smooth diffeomorphisms fixing each puncture $p_{i}$ together with a ray in its tangent space act transitively on the equivalence classes of paths $\gamma_{i}$ that look as in Figure 4 with respect to some smooth coordinate system on an open disc containing all the punctures. Restricting to a slightly smaller closed disc, this follows 
from the fact that smooth diffeomorphisms act transitively on the set of embeddings of a closed disc in the 2-sphere [36]. Then, having found a smooth diffeomorphism $f: S \rightarrow S$ which carries a given structure $\mathcal{S}$ to a given structure $\mathcal{S}^{\prime}$, one can use approximation methods to find a real-analytic diffeomorphism $g$ that also does this. In particular, by a version of the Weierstrass theorem, for any smooth diffeomorphism $f: S \rightarrow S$ one can find a polynomial $p: \mathbb{R}^{3} \rightarrow \mathbb{R}^{3}$ which, when restricted to $S$, is arbitrarily close to $f$ in the $C^{1}$ topology and has the same values and first derivatives as $f$ at a chosen finite set of points. Composing such a polynomial with radial projection to $S$ gives the desired realanalytic diffeomorphism $g: S \rightarrow S$.

Combining these results it follows that, for a given number $n$, all Hilbert spaces under consideration lie in the orbit of the induced action of $\mathcal{D}$; they are all physically equivalent. The quantum diffeomorphism constraint requires that we consider only one point in this orbit. Therefore, for each natural number $n$, we fix a specific structure $\mathcal{S}(\mathcal{P})$ on the horizon and construct the corresponding surface Hilbert space $\mathcal{H}_{S}^{\mathcal{S}(\mathcal{P}), a}$, which we denote as $\mathcal{H}_{S}^{n, a}$. Each state in this Hilbert space serves as the representative of all states (belonging to various Hilbert spaces associated with $n$ punctures) in its diffeomorphism equivalence class.

Next, let us consider the volume Hilbert spaces $\mathcal{H}_{V}^{\mathcal{P}, m}$, where $m_{i} \in$ $\left\{-j_{i},-j_{i}+1, \ldots, j_{i}\right\}$ and $j_{i}$ are the labels attached to the edges of the spin networks in the volume which puncture the horizon. Fix an integer $n$ and the corresponding punctures $p_{1}, \ldots, p_{n}$. We need to factor out by the action of diffeomorphisms which interchange these punctures. As far as the 'surface data' is concerned we can again use gauge fixing: We will only use ordered labels $j$ via $j_{1} \leq j_{2} \cdots \leq j_{n}$. Then, it remains to consider only the subgroup $\mathcal{D}_{S}$ of $\mathcal{D}$ consisting of diffeomorphisms in $\mathcal{D}$ which are identity on $S$. Our task is to construct volume states which are invariant under the action of this group. For this, one can essentially repeat the 'group averaging procedure' developed and used in $[18,23,24]$ for the case when $M$ has no internal boundaries. Each of these states corresponds to the equivalence class of spin network states in $\mathcal{H}_{V}^{\mathcal{P}, m}$ related to one another by a diffeomorphism in $\mathcal{D}_{S}$. Denote by $\mathcal{H}_{V}^{n, m}$ the resulting volume Hilbert space.

We can now display the total Hilbert space $\mathcal{H}^{\text {Diff }}$ of states which 
satisfy the diffeomorphism constraint:

$$
\mathcal{H}^{\text {Diff }}=\bigoplus_{n, m, a: 2 m=-a \bmod k} \mathcal{H}_{V}^{n, m} \otimes \mathcal{H}_{S}^{n, a} .
$$

Here the number of punctures $n$ ranges over positive integers; $m_{i}$ with $i=1,2, \ldots, n$ are half-integers with $m_{i} \in\left\{-j_{i},-j_{i}+1, \ldots, j_{i}\right\}$ where $j_{i}$ are ordered half-integers $j_{1} \leq j_{2} \leq \cdots \leq j_{n}$; and $a_{i}$ are nonzero elements of $\mathbb{Z}_{k}$ that sum to zero.

\subsubsection{Hamiltonian constraint}

In this subsection, we will impose the remaining, Hamiltonian constraint. In the literature, there exist some concrete proposals for defining this operator on the space of solutions to the diffeomorphism and Gauss constraints and for finding its kernel, i.e., the physical states (see, in particular, $[27,28]$.) However, as pointed out at the end of Section 2.2 , these proposals are still being examined in detail. Fortunately, our analysis of the geometry of quantum horizon and entropy calculation are largely insensitive to this freedom.

Let us begin by recalling the situation in the classical theory. To obtain a function on the phase space, the Hamiltonian constraint has to be smeared by a 'lapse field' $N$ on the 3-manifold $M$. The resulting function is differentiable on the phase space, i.e., defines a Hamiltonian vector field, only if the $N$ tends to zero both at infinity and the horizon. Motions along the resulting Hamiltonian vector field are 'bubble timeevolutions'; in the space-time picture, the 3-manifold is kept fixed both at infinity and at the horizon. These motions are to be treated as 'gauge' and the phase space of physical states is obtained by quotienting the full phase space by the orbits of these Hamiltonian vector fields, or, by picking a cross-section in the phase space which intersects each orbit once and only once. (True dynamics corresponds to time evolution in which the lapse $N$ is not constrained to vanish on the horizon and infinity and is generated by a genuine Hamiltonian function on the physical phase space.) Finally, consider the function $A_{S}$ on the phase space which measures the horizon area. Because the lapse $N$ smearing the Hamiltonian constraint goes to zero at the horizon, $A_{S}$ is constant on the gauge orbits and therefore defines an observable on the physical phase space. 
Let us now turn to the quantum theory. To impose the Hamiltonian constraint, we must smear the appropriately constructed constraint operator by a lapse which goes to zero on $S$. Therefore, the constraint leaves the surface states untouched and has non-trivial action only on volume states. Consider the subspace $\mathcal{H}_{V}^{n, m}$ of volume states in (39). Imposition of the Hamiltonian constraint picks out a subspace $\tilde{\mathcal{H}}_{V}^{n, m}$ of $\mathcal{H}_{V}^{n, m}$, which again carries the labels $n, m$ because they refer to punctures on $S$ and the lapse vanishes on $S$. Thus, the total physical Hilbert space is now given by:

$$
\mathcal{H}^{\text {Phys }}=\bigoplus_{n, m, a: 2 m=-a \bmod k} \tilde{\mathcal{H}}_{V}^{n, m} \otimes \mathcal{H}_{S}^{n, a}
$$

where $n$ ranges over natural numbers, $m$ ranges over $n$-tuples of halfintegers, and $a$ ranges over $n$-tuples of nonzero elements of $\mathbb{Z}_{k}$ that sum to zero.

As in the classical theory, the area observable $\hat{A}_{S}$ is well-defined on physical states. To show this let us proceed step by step, starting with the kinematical Hilbert space $\mathcal{H}^{\text {Kin }}$. Recall from Section 4.2 that each spin network state in $\mathcal{H}^{\text {Kin }}$ is an eigenstate of the area operator $\hat{A}_{S}$ and the eigenvalue depends only on the spin labels at the punctures. Therefore, the diffeomorphism-invariant state that results from group averaging of any spin network state is again an eigenstate with the same property. Thus $\hat{A}_{S}$ has a well-defined action on $\mathcal{H}^{\text {Diff }}$. Finally, the action descends to $\mathcal{H}^{\text {Phys }}$ because each $\tilde{\mathcal{H}}_{V}^{n, m}$ is a subspace of $\mathcal{H}_{V}^{n, m}$.

The following description of the physical Hilbert space will be useful in the entropy calculation of the next section. Define the 'physical volume Hilbert space' as follows:

$$
\mathcal{H}_{V}^{\text {Phys }}=\bigoplus_{n, m} \tilde{\mathcal{H}}_{V}^{n, m}
$$

where $n$ ranges over natural numbers and $m$ ranges over $n$-tuples of nonzero half-integers. Similarly, define the 'physical surface Hilbert space' by:

$$
\mathcal{H}_{S}^{\text {Phys }}=\bigoplus_{n, a} \mathcal{H}_{S}^{n, a}
$$

where $n$ ranges over natural numbers and $a$ ranges over $n$-tuples of nonzero elements of $\mathbb{Z}_{k}$ that sum to zero. By equation (40) we have

$$
\mathcal{H}^{\text {Phys }} \subset \mathcal{H}_{V}^{\text {Phys }} \otimes \mathcal{H}_{S}^{\text {Phys }} \text {. }
$$


This decomposition allows us to isolate the volume and surface degrees of freedom of the black hole system. In the next section we will use this decomposition to compute the entropy of a non-rotating black hole whose event horizon has its area lying in a given range.

\section{Entropy}

We now have all the machinery needed for the computation of entropy of isolated horizons. Recall first that our fundamental macroscopic parameters are defined intrinsically at the horizon, without reference to infinity: they are area, angular momentum and charges associated with matter fields. (In this framework, mass is a secondary quantity, expressed as a specific function of the fundamental ones.) In the nonrotating case now under consideration, it is then natural to begin with a microcanonical ensemble consisting of physical quantum states which endow the horizon $S$ with an area lying in a small interval containing a fixed value $a_{0}$ and charges lying in a small interval containing fixed values $Q_{0}$ and count the independent surface states in the ensemble. We will carry out this task in three steps. The first will focus on pure general relativity where the only relevant horizon parameter is the area $a_{0}$. In Section 6.1 we outline the strategy and in Section 6.2 we carry out the desired counting in detail. Matter fields and charges are introduced in the third step. This step is carried out in Section 6.3, which also summarizes the current viewpoint towards the final result. Further remarks on the physical meaning of this entropy and the relation of our calculation to those carried out in other approaches can be found in Section 7.

\subsection{Strategy}

Consider isolated horizons whose area $a$ lies in the range $a_{0}-\delta \leq a \leq$ $a_{0}+\delta$. Since we are only interested in the entropy of the horizon itself, not the surrounding spacetime, we start by considering all physical states for which the horizon area lies in this range, and then trace out over the volume states to obtain a density matrix $\rho_{\text {bh }}$ on $\mathcal{H}_{S}^{\text {phys }}$ describing a maximal-entropy mixture of surface states for which the 
horizon area lies in this range. The statistical mechanical entropy of this mixture will then be given by

$$
S_{\mathrm{bh}}=-\operatorname{Tr}\left(\rho_{\mathrm{bh}} \ln \rho_{\mathrm{bh}}\right) .
$$

In more detail, first recall from Section 5.2.3 that we can define the area operator $\hat{A}_{S}$ as an operator on the physical Hilbert space $\mathcal{H}^{\text {phys }}$. Let $\mathcal{H}^{\text {bh }} \subset \mathcal{H}^{\text {phys }}$ be the subspace spanned by eigenstates of $\hat{A}_{S}$ with eigenvalues $a$ lying in the range $a_{0}-\delta \leq a \leq a_{0}+\delta$. Because we allow for the presence of arbitrary radiation in the bulk, the space $\mathcal{H}^{\text {bh }}$ will be infinite-dimensional, so it will be impossible to normalize the projection onto this subspace to obtain a density matrix on $\mathcal{H}^{\text {phys }}$. However, using the decomposition given in equation (43), we can write any vector $\psi \in \mathcal{H}^{\text {bh }}$ in the form

$$
\psi=\sum_{i} \psi_{V}^{i} \otimes \psi_{S}^{i}
$$

where $\psi_{V}^{i}$ lie in the physical volume Hilbert space and $\psi_{S}^{i}$ lie in the physical surface Hilbert space. In particular, there is a smallest subspace of $\mathcal{H}_{S}^{\text {phys }}$, which we call $\mathcal{H}_{S}^{\text {bh }}$, with the property that any vector in $\mathcal{H}^{\text {bh }}$ can be written as above with $\psi_{S}^{i} \in \mathcal{H}_{S}^{\text {phys }}$. States in $\mathcal{H}_{S}^{\text {bh }}$ describe the surface degrees of freedom of states in $\mathcal{H}^{\text {bh }}$. The space $\mathcal{H}_{S}^{\text {bh }}$ is finite-dimensional (as we shall soon see), so we may normalize the projection from $\mathcal{H}_{S}$ onto $\mathcal{H}_{S}^{\text {bh }}$ to to obtain a density matrix $\rho_{\text {bh }}$. This density matrix describes the maximal-entropy mixed state of the surface geometry compatible with the constraint $a_{0}-\delta \leq a \leq a_{0}+\delta$. The entropy of this state is

$$
S_{\mathrm{bh}}=\ln N_{\mathrm{bh}}
$$

where $N_{\mathrm{bh}}$ is the dimension of the space $\mathcal{H}_{S}^{\mathrm{bh}}$, i.e., the number of physical surface states compatible with the above constraint on the horizon area. To compute this entropy, we thus need to count states forming a basis of $\mathcal{H}_{S}^{\text {bh }}$.

To do this count we first need some definitions. Given an ordered list of positive half-integers $j=\left(j_{1}, \ldots, j_{n}\right)$, let $A(j)$ be the corresponding eigenvalue of the area operator:

$$
A(j)=8 \pi \gamma \ell_{P}^{2} \sum_{i} \sqrt{j_{i}\left(j_{i}+1\right)} .
$$

We say the list $j$ is 'permissible' if it satisfies

$$
j_{1} \leq j_{2} \ldots \leq j_{n} \quad \text { and } \quad a_{0}-\delta \leq A(j) \leq a_{0}+\delta .
$$


Given a list of half-integers $\left(m_{1}, \ldots, m_{n}\right)$, we say it is 'permissible' if for some permissible list of positive half-integers $j=\left(j_{1}, \ldots, j_{n}\right)$ we have $m_{i} \in\left\{-j_{i},-j_{i}+1, \ldots, j_{i}\right\}$. Finally, given a list $\left(a_{1}, \ldots, a_{n}\right)$ of elements of $\mathbb{Z}_{k}$, we say it is 'permissible' if $a_{1}+\cdots+a_{n}=0 \bmod k$ and $a_{i}=-2 m_{i}$ $\bmod k$ for some permissible list of half-integers $\left(m_{1}, \ldots, m_{n}\right)$.

Given these definitions, we may determine the dimension of $\mathcal{H}_{S}^{\text {bh }}$ with the help of a weak assumption on the nature of the Hamiltonian constraint. We assume that for any permissible lists $j, m$ there exists at least one state $\psi_{V} \in \mathcal{H}_{V}^{\mathcal{P}, j} \cap \mathcal{H}_{V}^{\mathcal{P}, m}$ that is annihilated by the Hamiltonian constraint. Given this assumption, the dimension of $\mathcal{H}_{S}^{\text {bh }}$ is exactly the number of permissible lists $a$ of elements of $\mathbb{Z}_{k}$. (If this assumption fails, the dimension of $\mathcal{H}_{S}^{\text {bh }}$ will be smaller, so it is finite in any event.)

In the following section we use this description of the dimension of $\mathcal{H}_{S}^{\text {bh }}$ to establish upper and lower bounds on it which enable us to show that for $\delta$ sufficiently large, but still on the order of $\ell_{P}^{2}$,

$$
S_{\mathrm{bh}}=\frac{\ln 2}{4 \pi \sqrt{3} \gamma \ell_{P}^{2}} a_{0}+o\left(a_{0}\right)
$$

Here ' $o\left(a_{0}\right)$ ' refers to a quantity which, divided by $a_{0}$, approaches zero in the limit $a_{0} \rightarrow \infty$. Thus, our result agrees with Hawking's semiclassical calculation [37] in the sector of the quantum theory on which the Barbero-Immirzi parameter $\gamma$ equals $\gamma_{0}$ with

$$
\gamma_{0}=\frac{\ln 2}{\pi \sqrt{3}} .
$$

We will return to this point in Section 6.3.

A quick sketch of the calculation in the following section reveals its physical significance. The count of black hole horizon states is dominated by states in which all the spin network edges piercing the horizon are labelled by spins $j_{i}=1 / 2$. Each such edge contributes $8 \pi \gamma \ell_{P}^{2} \sqrt{j_{i}\left(j_{i}+1\right)}=4 \pi \sqrt{3} \gamma \ell_{P}^{2}$ to the area of the event horizon, so the total number of spin network edges puncturing the horizon is approximately $a_{0} / 4 \pi \sqrt{3} \gamma \ell_{P}^{2}$. Moreover, when $j_{i}=1 / 2$ there are 2 allowed values of $a_{i}$, namely \pm 1 , corresponding to angle deficits of $\pm 4 \pi / k$ for the Levi-Civita connection at the puncture $p_{i}$. It follows that each such puncture contributes $\ln 2$ to the black hole entropy. The total entropy of the black hole is therefore asymptotic to $\left(\ln 2 / 4 \pi \sqrt{3} \gamma \ell_{P}^{2}\right) a_{0}$. 


\subsection{Counting}

A lower bound on $N_{\text {bh }}$ can be obtained as follows. Let us consider lists of positive half-integers $j=\left(j_{1}, \ldots, j_{n}\right)$ in which all the $j_{i}$ are equal to $1 / 2$. The area corresponding to such a list is

$$
A(j)=4 \pi \sqrt{3} \gamma \ell_{P}^{2} n \text {. }
$$

Thus if

$$
\delta>8 \pi \sqrt{3} \gamma \ell_{P}^{2}
$$

one can always find an even integer $n$ such that (44) is satisfied, giving us a permissible list $j$. From this choice of $j$ we obtain $2^{n}$ permissible lists $m=\left(m_{1}, \ldots, m_{n}\right)$, since each $m_{i}$ can be either $1 / 2$ or $-1 / 2$. Since $n$ is even, $\left(\begin{array}{c}n \\ n / 2\end{array}\right)$ of these lists satisfy $m_{1}+\cdots+m_{n}=0$, and thus give rise to admissible lists $a$ of elements of $\mathbb{Z}_{k}$ via $a_{i}=-2 m_{i} \bmod k$. When $k>2$, which is certainly true when the black hole area is large in Planck units, all of these admissible lists $a$ are distinct.

In short, given that $\delta$ satisfies (45) and $a_{0}$ is large enough, we have the lower bound

$$
N_{\text {bh }} \geq\left(\begin{array}{c}
n \\
n / 2
\end{array}\right)
$$

where

$$
a_{0}-\delta \leq 4 \pi \sqrt{3} \gamma \ell_{P}^{2} n \leq a_{0}+\delta .
$$

Using Stirling's formula, one can show that for large $n$ we have

$$
\left(\begin{array}{c}
n \\
n / 2
\end{array}\right) \sim 2^{n+1 / 2} / \sqrt{\pi n}
$$

We thus have

$$
S_{\mathrm{bh}}=\ln N_{\mathrm{bh}} \geq \ln \left(2^{n}\right)-o(n)
$$

for large $n$, where ' $o(n)$ ' refers to a quantity which, divided by $n$, approaches zero as $n \rightarrow \infty$. It follows that

$$
S_{\mathrm{bh}} \geq \frac{\ln 2}{4 \pi \sqrt{3} \gamma \ell_{P}^{2}} a_{0}-o\left(a_{0}\right) .
$$

Next we obtain an upper bound on $N_{\mathrm{bh}}$. We certainly have

$$
N_{\mathrm{bh}} \leq \sum_{j} d(j)
$$


where we sum over all permissible lists $j=\left(j_{1}, \ldots, j_{n}\right)$ of positive halfintegers, and $d(j)$ is the number of permissible lists $m$ of half-integers with $m_{i} \in\left\{-j_{i},-j_{i}+1, \ldots, j_{i}\right\}$, namely:

$$
d(j)=\prod_{i=1}^{n}\left(2 j_{i}+1\right)
$$

To get a more explicit upper bound, we introduce the 'density of states'

$$
g(A)=\sum_{j} d(j) \delta(A-A(j))
$$

where we sum over all lists $j$ of positive half-integers. By equation (47) we clearly have

$$
N_{\mathrm{bh}} \leq \int_{a_{0}-\delta}^{a_{0}+\delta} g(A) d A
$$

To make further progress, we need an upper bound on the rate of growth of the density of states as $A \rightarrow \infty$. To do this, we introduce a 'partition function', which is the Laplace transform of the density of states:

$$
Z(\alpha)=\int_{0}^{\infty} e^{-\alpha A} g(A) d A .
$$

Here $\alpha$ represents an intensive variable conjugate to the horizon area. This integral converges when the real part of $\alpha$ is sufficiently large, but blows up when the damping factor $e^{-\alpha A}$ is insufficient to counteract the growth of $g(A)$, so the function $Z$ is analytic on a half-plane of the form $\operatorname{Re}(\alpha)>\alpha_{0}$. As we shall see, it extends to a meromorphic function on the half-plane $\operatorname{Re}(\alpha)>0$. The poles with the largest real part occur when $\operatorname{Re}(\alpha)=\alpha_{0}$, i.e., exactly at the point where the integral defining $Z(\alpha)$ ceases to converge. In what follows, we use this to put an upper bound on the growth of the density of states.

Since $g$ is positive, for $\alpha>0$ we have

$$
\begin{aligned}
\int_{a_{0}-\delta}^{a_{0}+\delta} g(A) d A & \leq \int_{0}^{a_{0}+\delta} g(A) d A \\
& \leq \int_{0}^{a_{0}+\delta} e^{a_{0}+\delta-A} g(A) d A \\
& \leq e^{\alpha\left(a_{0}+\delta\right)} Z(\alpha)
\end{aligned}
$$


so by equation (48) we have

$$
N_{\mathrm{bh}} \leq e^{\alpha\left(a_{0}+\delta\right)} Z(\alpha)
$$

whenever the integral defining $Z(\alpha)$ converges.

A simple calculation shows that

$$
Z(\alpha)=\sum_{j} e^{-\alpha A(j)} d(j)
$$

where we sum over all lists $j$ of positive half-integers (with $j_{1} \leq j_{2} \leq$ $\left.\ldots \leq j_{n}\right)$. By the definition of $d(j)$ we have

$$
Z(\alpha)=\sum_{n} \sum_{j_{1} \leq j_{2} \ldots \leq j_{n}} e^{-\alpha\left[8 \pi \gamma \ell_{P}^{2} \sum_{i=1}^{n} \sqrt{j_{i}\left(j_{i}+1\right)}\right]} \prod_{i=1}^{n}\left(2 j_{i}+1\right)
$$

and this in turn implies

$$
Z(\alpha)=\prod_{l} \frac{1}{1-(2 l+1) e^{-\alpha\left[8 \pi \gamma \ell_{P}^{2} \sqrt{l(l+1)}\right]}} .
$$

where we take the product over positive half-integers $l$. Note that each factor in this infinite product is analytic as a function of $\alpha$ everywhere except for a simple poles where the denominator vanishes, namely at the points

$$
\alpha=\frac{\ln (2 l+1)+2 \pi i n}{8 \pi \gamma \ell_{P}^{2} \sqrt{l(l+1)}} .
$$

Note also that for any fixed choice of $\alpha$ with $\operatorname{Re}(\alpha)>0$, the terms in the infinite product rapidly approach 1 as $j \rightarrow \infty$. It follows that $Z(\alpha)$ is analytic on the right half-plane except for simple poles at the above points. The poles of $Z(\alpha)$ with the largest real part occur when $l=1 / 2$, so $Z(\alpha)$ is analytic for

$$
\operatorname{Re}(\alpha)>\alpha_{0}=\frac{\ln 2}{4 \pi \sqrt{3} \gamma \ell_{P}^{2}}
$$

and $Z(\alpha)$ has a simple pole at $\alpha_{0}$.

It follows that for $\alpha>\alpha_{0}$ the integral defining $Z(\alpha)$ converges, and we have

$$
Z(\alpha) \leq \frac{K}{\alpha-\alpha_{0}}
$$


for some constant $K$ when $\alpha$ is close to $\alpha_{0}$. Equation (49) thus gives

$$
N_{\mathrm{bh}} \leq \frac{K}{\alpha-\alpha_{0}} e^{\alpha\left(a_{0}+\delta\right)}
$$

Taking the logarithm of both sides, we obtain

$$
S_{\mathrm{bh}} \leq \alpha\left(a_{0}+\delta\right)+\ln \left(\frac{K}{\alpha-\alpha_{0}}\right)
$$

so that as $a_{0} \rightarrow \infty$ we have

$$
S_{\mathrm{bh}} \leq \frac{\ln 2}{4 \pi \sqrt{3} \gamma \ell_{P}^{2}} a_{0}+o\left(a_{0}\right) .
$$

Combining the upper bound (50) with the lower bound (46), we obtain

$$
S_{\mathrm{bh}}=\frac{\ln 2}{4 \pi \sqrt{3} \gamma \ell_{P}^{2}} a_{0}+o\left(a_{0}\right)
$$

whenever $\delta$ is sufficiently large, as in (45). This formula asymptotically matches the Bekenstein-Hawking formula:

$$
S_{\mathrm{bh}}=\frac{1}{4} \frac{a_{0}}{\ell_{P}^{2}}
$$

if we take the Barbero-Immirzi parameter $\gamma$ to equal

$$
\gamma_{0}=\frac{\ln 2}{\pi \sqrt{3}}
$$

This concludes the mathematics of counting states. We comment further on the final result at the end of Section 6.3 and also in Section 7.

\subsection{Charged Black Holes}

Using Hamiltonian methods, laws of black hole mechanics have been extended to isolated horizons for general relativity coupled to Maxwell, dilaton, and Yang-Mills fields [2,3]. In all cases, the surface gravity $\kappa$ is constant on isolated horizons and the change in the horizon energy $E_{S}$ 
is related to the changes in its parameters via: $\delta E_{S}=(\kappa / 8 \pi G) \delta a_{S}+$ work terms. Comparison with the familiar zeroth and first laws of thermodynamics suggests that, as in standard black hole mechanics, one should assign an isolated horizon a temperature $T=(\kappa \hbar / 2 \pi)$ and an entropy $S=\left(a_{S} / 4 G \hbar\right) \equiv\left(a_{S} / 4 \ell_{P}^{2}\right)$. In particular, these semi-classical considerations suggest that the entropy is purely geometrical: it is a multiple of the area of the horizon, irrespective of the values of matter fields and their charges at the horizon. A statistical mechanical derivation of entropy has to account for this key fact.

For simplicity, in this section, we will restrict ourselves to Maxwell and dilaton fields: the inclusion of Yang-Mills fields only adds certain technical complications without altering the essence of our arguments or the final results. (If the dilaton field vanishes, we are left with the Einstein-Maxwell system.) Let us then begin by briefly recalling the relevant aspects of the classical theory of the Einstein-Maxwell-dilaton system from $[1,2,3]$. In this theory, the isolated horizon carries four parameters which can be taken to be the area $a_{0}$, electric charge $Q_{0}$, the magnetic charge $P_{0}$ and the value of the dilaton field $\phi_{0}$, all defined intrinsically on the horizon. With suitable boundary conditions, the action principle for the total system is well-defined if we simply add the standard matter action terms to the gravitational action of [1]. In particular, there is no need to introduce any new surface terms at the horizon in the matter sector. One can then pass to the phase space through the standard Legendre transform. The total phase space $\mathcal{X}_{\text {total }}$ has three striking features which are important for quantization.

First, the gravitational part $\mathcal{X}_{\text {grav }}$ of the phase space is exactly the same as $\mathcal{X}$ of Section 2. In particular, the key boundary condition (6) constraining the gravitational phase space variables $(A, \Sigma)$ at the horizon is left unaltered in spite of the presence of matter fields on the horizon itself. This is remarkable because the presence of matter does modify the curvature $F$ of $A$ : while in absence of matter, $F$ is determined by the Weyl tensor (technically the Newman-Penrose component $\Psi_{2}$ at the horizon), in presence of matter it depends also on the Ricci curvature (the $\Phi_{11}$ component and the scalar curvature). However, because of the isolated horizon boundary conditions, the pullback of $F$ to $S$ is always proportional to the pullback of $\Sigma$, and the Gauss-Bonnet theorem implies that the proportionality factor is always $-2 \pi / a_{0}$ irrespective of the value of the Ricci curvature at the horizon. Furthermore, 
as one might expect, the gravitational part of the symplectic structure is unaffected by the presence of matter. These properties are important to our analysis because they imply that the description of the quantum horizon geometry developed in Sections 4 and 5 continues to be valid, without any change whatsoever, in the presence of matter fields.

The matter sector of the phase space consists of fields $(\phi, \pi ; \mathbf{A}, \mathbf{\Pi})$ on the spatial 3-manifold $M$, satisfying appropriate boundary conditions. Here $\phi$ is the dilaton scalar field, the 3 -form $\pi$ is its conjugate momentum, $\mathbf{A}$ is the electromagnetic vector potential, and $\boldsymbol{\Pi}$ is its conjugate momentum, related to the electric field 2-form $\mathbf{E}$ via $\Pi=\exp (-2 \alpha \phi) \mathbf{E}$. (Here $\alpha$ is the dilaton coupling constant.) We denote the curvature of A - the magnetic field 2-form- by $\mathbf{F}$. The boundary conditions at infinity are the standard ones. The gravitational boundary conditions at the horizon imply that the dilaton field $\phi$ is constant on $S$. We will denote its value by $\phi_{0}$. The remaining horizon parameters can be taken to be the electric and magnetic charges,

$$
P_{0}:=\frac{1}{4 \pi} \oint_{S} \mathbf{F} \quad \text { and } \quad Q_{0}:=\frac{1}{4 \pi} \oint_{S} \mathbf{E} .
$$

All three quantities are conserved in time. The remaining horizon boundary conditions, relevant for quantization and entropy, are:

$$
\phi=\phi_{0}, \quad \underline{\mathbf{F}}=2 P_{0} d W, \quad \text { and } \quad \underline{\mathbf{E}}=2 Q_{0} d W
$$

where, as before, underbars denote pullbacks to $S$ and $W$ denotes the $U(1)$ gravitational connection. Thus, given the horizon parameters, the local values of matter fields on the horizon are completely determined by the gravitational connection already at the kinematical level, without reference to the bulk equations of motion. This is the second important feature. It already provides a partial explanation of why the entropy is purely geometrical.

The third important feature of the Hamiltonian framework is the form of the matter symplectic structure. It is given by:

$$
\Omega_{\mathrm{matter}}(\phi, \pi ; \delta \mathbf{A}, \delta \mathbf{\Pi})=\int_{M}(\phi \pi+\mathbf{A} \wedge \mathbf{\Pi}) .
$$

The symplectic structure on the full phase space is simply $\Omega_{\text {total }}=$ $\Omega_{\text {grav }}+\Omega_{\text {matter }}$, the gravitational symplectic structure being given by 
(7). Note that, unlike the $\Omega_{\text {grav }}$, the matter contribution $\Omega_{\text {matter }}$ does not contain a surface term. This difference has major ramifications for quantization.

For, in the gravitational sector, the presence of a surface term in $\Omega_{\text {grav }}$ led us naturally to construct a bulk phase space $\mathcal{X}_{V}$ and a surface phase space $\mathcal{X}_{S}$ with projections $p_{V}$ and $p_{S}$ from the gravitational phase space $\mathcal{X}_{\text {grav }}$ to $\mathcal{X}_{V}$ and $\mathcal{X}_{S}$ respectively. (See the remark at the end of Section 2). This in turn suggested that in quantum theory we should begin with a total Hilbert space $\mathcal{H}$ of the form $\mathcal{H}_{V} \otimes H_{S}$. The volume states were obtained by quantization of the volume phase space $\mathcal{X}_{V}$ and the surface states by quantization of $\mathcal{X}_{S}$. In the matter sector, on the other hand, there is no surface term in $\Omega_{\text {matter }}$. Therefore, the matter sector has to be quantized differently: now only the volume Hilbert space can be constructed naturally. Furthermore, as argued in Section 6.1, the details of even this construction are not needed in the entropy calculation which only involves counting of surface states in the microcanonical ensemble. The fact that the only independent surface states come from gravity explains, in this approach, the purely geometric origin of entropy.

What happens then to the matter boundary conditions (54) in the quantization procedure? These are incorporated by eliminating the surface Maxwell fields $\underline{\mathbf{F}}$ and $\underline{\mathbf{E}}$ in favor of $\left(1 / P_{0}\right) d W$ and $\left(1 / Q_{0}\right) d W$ respectively prior to quantization. Thus, any classical observable involving the surface values of the Maxwell fields becomes, in quantum theory, an operator on the gravitational surface Hilbert space. All horizon surface physics in the Einstein-Maxwell-dilaton system occurs only on the gravitational Hilbert space $\mathcal{H}_{S}$ constructed in Sections 4 . Hence, the counting argument of Section 6.2 is unaltered and entropy of an isolated horizon is independent of the values of the charges $P_{0}, Q_{0}$ and $\phi_{0}$. For large values of $a_{0} / \ell_{P}^{2}$ the leading term in the formula for the entropy is always given by (51). Thus, all the surface degrees of freedom associated with a quantum isolated horizon belong to the gravitational sector alone. In more physical terms, the quantum fluctuations of matter fields on the horizon manifest themselves only through the quantum fluctuations of the horizon geometry. ${ }^{5}$

\footnotetext{
${ }^{5}$ It is interesting to consider an alternate, hypothetical strategy. Although there is no natural way to construct the surface Hilbert space for the Maxwell theory, suppose one just chose, in an ad hoc manner, a quantization procedure without
} 
The fact that the operators corresponding to surface matter fields act on the gravitational Hilbert space $\mathcal{H}_{S}$ may seem somewhat counterintuitive at first. This arose because, already at the kinematical level, we were naturally led to put all the degrees of freedom of the coupled system in the gravitational sector. A qualitatively similar situation occurs in quantum gravity coupled to the Maxwell (or scalar) field in $2+1$ dimensions. The rotationally symmetric sector of this system provides an exactly soluble quantum field theory with an infinite number of degrees of freedom [38]. In this theory, it is natural to arrange matters such that the true degrees of freedom reside in the Maxwell field which, furthermore, can be quantized readily. The gravitational observables, such as the fully gauge fixed metric, are then operators on the Maxwell Hilbert space. If one constructed a microcanonical ensemble in this theory using some Maxwell and gravitational -or, indeed, even purely gravitational - observables, the state counting would still be carried out on the appropriate sector of the pure Maxwell theory. (Unfortunately, however, this theory does not admit black holes and so one can not use this procedure to shed light on black hole entropy.) In the present case, the situation is analogous, although the roles of the gravitational and matter fields are now reversed and the reasoning is not tied to the details of dynamics. (For further remarks on the issue of dynamics, see Section 7.)

We conclude with a few remarks on the results obtained in Section 6 as a whole.

1. Extremal black holes. For simplicity, let us consider the EinsteinMaxwell system without dilatons. ${ }^{6}$ In this case, we were led to eliminate the surface matter fields in favor of the surface gravitational field be-

first solving for the surface Maxwell fields in terms of the gravitational $d W$. Then, one would be led to impose the boundary conditions (54) as operator restrictions on permissible quantum states. How would one then see that there are no independent Maxwell surface states? Setting $F=d W$ as in Section 3, the equation constraining Maxwell surface states would then $\operatorname{read}\left(\Psi_{\text {grav }, V} \otimes \Psi_{\text {grav }, S}\right) \otimes\left(\Psi_{\text {Max }, V} \otimes\right.$ $\left.\exp (i \underline{\hat{\mathbf{F}}}) \Psi_{\mathrm{Max}, S}\right)=\left(\Psi_{\mathrm{grav}, V} \otimes \exp \left(2 i P_{0} \hat{F}\right) \Psi_{\mathrm{grav}, S}\right) \otimes\left(\Psi_{\mathrm{Max}, V} \otimes \Psi_{\mathrm{Max}, S}\right)$. Because of this tight correlation, the entropy calculation would again reduce to the counting only of independent gravitational surface states.

${ }^{6}$ In this remark we are interested in extremal black holes and the horizon geometry is non-singular in extremal static solutions only when the dilatonic coupling constant is zero, i.e., only in the Einstein-Maxwell sector of the theory. Inclusion of a dilaton therefore adds unnecessary complications to this discussion. 
cause of three key factors. First, the gravitational boundary condition (6) could be expressed entirely in terms of the parameter $a_{0}$ without any reference to charges $Q_{0}$ and $P_{0}$. Second, the surface term in the total symplectic structure $\Omega_{\text {grav }}$ is also purely gravitational. Finally, while the gravitational boundary conditions (6) made no reference to matter fields, the gravitational curvature $d W$ plays a key role in the matter boundary conditions (54). Therefore, at the kinematical level, while it was possible to first quantize the gravitational sector without any reference to the values of electromagnetic charges, it was not possible to reverse the strategy and first quantize the matter fields. However, if we restrict ourselves only to the extremal, static black holes, we can express the area $a_{0}$ in terms of the electromagnetic charges. Furthermore, in this case, the Weyl tensor vanishes identically on the horizon whence the gravitational curvature $d W$ is completely expressible in terms of the electromagnetic field. Therefore, it is quite possible that the surface term in the symplectic structure could also be recast in terms only of the electromagnetic field. If this can be done, it should be possible to reverse the strategy, transfer the gravitational surface degrees of freedom to the electromagnetic ones and compute the entropy purely in terms of the electromagnetic surface states. This calculation would be instructive and may well enable one to relate the present approach to that based on string theory.

2. The Barbero-Immirzi parameter. We have shown that, irrespective of the values of matter charges, entropy of a non-rotating isolated horizon is proportional to its area $a_{0}$. In this calculation, the proportionality factor depends on the value of the Barbero-Immirzi parameter $\gamma$ simply because the elementary quantum of area depends on this value. This is an inherent feature of the approach: just as there is the $\theta$-ambiguity in QCD, we have a 1-parameter family of quantization ambiguities, labelled by $\gamma$, and in general physical predictions depend on which $\gamma$-sector of the quantum theory one chooses. In QCD, one can hope to make a single judicious experiment to fix the value of $\theta$; then all further experiments can test the theory. The situation here is rather similar. If we could somehow make a judicious experiment to measure the value of, e.g., the area quantum, the value of $\gamma$-and hence the quantum theory of geometry- would be fixed. Although such a direct measurement seems completely out of reach at present, one can regard the entropy calculation as giving an indirect handle on the elementary quantum of area, thereby selecting the value of $\gamma$ realized in Nature. 
More precisely, one can adopt the following 'phenomenological approach.' In the classical theory, all $\gamma$ sectors are related simply by a canonical transformation and hence completely equivalent to one another. However, as with the $\theta$-sectors of QCD, they lead to different physical predictions as soon as $\hbar$ is non-zero, i.e. already at the semiclassical level. In particular, although the non-perturbative quantum theories corresponding to various $\gamma$ values are internally consistent, they would not all agree with the standard semi-classical calculations performed in the framework of quantum field theory in curved spacetimes. One can thus hope to constrain the value of $\gamma$ by demanding that for large black holes, predictions of the full, non-perturbative quantum theory should reduce to those of the standard quantum field theory in curved spacetimes.

Since the semi-classical calculations have been performed for general relativity coupled to a variety of matter fields, a very large family of consistency checks is thus available. We could consider the isolated horizon in a de Sitter spacetime, or of an uncharged Schwarzschild black hole, or of a black hole with specific electric, magnetic and dilatonic charges. Now, it turned out that the requirement that the leading term in full quantum answer agree with the semi-classical result for any one of these horizons already fixes the value of $\gamma$ uniquely; $\gamma=$ $\gamma_{0}=\ln 2 / \pi \sqrt{3}$. This in turn fixes the theory. Then one can ask: Does one obtain the correct answer in all other contexts? We saw that the answer is in the affirmative. There is an infinite-dimensional space of spacetimes admitting non-rotating isolated horizons and in all these cases, the statistical mechanical entropy agrees to leading order with the Hawking-Bekenstein formula in the $\gamma_{0}$ sector. Thus, a very large number of consistency checks are met within the current quantum geometry approach.

We should emphasize, however, that what we have summarized here is only the present 'working viewpoint'. A more definitive stand would emerge only after a number of other semi-classical checks are made and the relation between the non-perturbative theory based on quantum geometry and more conventional perturbative quantum field theories in the continuum is better understood.

3. Wheeler's 'It from Bit'. The detailed calculation of Section 6.2 shows that the dominant contribution to entropy comes from states 
in which there is a very large number of punctures, each labelled by $j=\frac{1}{2}$ and $a= \pm 1$. Thus, there is a precise sense in which the quantum geometry calculation realizes John Wheeler's 'It from Bit' picture of the origin of black hole entropy [39] based on qualitative considerations from information theory.

4. Robustness of the calculation. Isolated horizon boundary conditions imply that the independent information in the gravitational connection $A$ at the boundary $S$ is coded in the $\mathrm{U}(1)$ connection $W$ (in the sense made explicit in Section 3.C of [1]). Therefore, in our analysis, surface states were described by the U(1) Chern-Simons theory. However, even if one ignores this fact and just uses $\mathrm{SU}(2)$ Chern-Simons theory to describe surface states, the leading order contribution to the entropy again turns out to be given by equation (51) [40, 41]. This precise agreement seems mysterious and it is important to understand systematically if this robustness could have been predicted on general grounds. If so, one may be able to relate our framework to conformal field theories that feature in other approaches [42]. Furthermore, there is already an elegant derivation of the sub-leading correction to entropy in the $\mathrm{SU}(2)$ framework [43] and the resulting logarithmic correction is supported by a closer analysis of the Cardy formula [44].

As another example of the robustness of the entropy calculation, note that the $j$ values that determine the black hole area via equation (20) are considered as volume rather than surface degrees of freedom in our approach. This arises naturally from the fact that the area operator is built using the $\Sigma$ field, which acts as an operator on the volume Hilbert space. But one might still wonder: if we decided to treat these $j$ values as surface degrees of freedom for the purposes of computing black hole entropy, how would the entropy formula be affected? The answer is that this would not affect the leading-order term in the entropy calculation. More precisely, suppose we redefined $N_{\text {bh }}$ to be the number, not of permissible lists $\left(a_{1}, \ldots, a_{n}\right)$, but of permissible lists $\left(j_{1}, \ldots, j_{n}\right)$ together with choices for each spin $j_{i}$ of an element $a_{i} \in \mathbb{Z}_{k}$ satisfying the conditions described in Section 6.1. Then we would still obtain the asymptotic formula (51) for the entropy $S_{\mathrm{bh}}=\ln N_{\mathrm{bh}}$. 


\section{Discussion}

This is the second paper in a series. The first paper [1] developed the classical Hamiltonian framework for the sector of general relativity consisting of spacetimes admitting a non-rotating isolated horizon as their inner boundary. Using this framework as the point of departure, in the first part of this paper we extended the quantum theory of geometry to describe the geometry of quantum horizons. The approach is rather general: we could use a single framework to incorporate isolated black hole horizons with no restrictions on values of electromagnetic charges or on the ratio of the horizon area to the cosmological constant. Furthermore, the cosmological horizons of the type discussed in [4] are incorporated from the beginning [2]. In the second part of the paper, we constructed a microcanonical ensemble consisting of spacetimes whose isolated horizons have areas and charges lying in small intervals around classical values, $a_{0}, Q_{0}, \ldots$ (with $a_{0} \gg \ell_{P}^{2}$ ), and calculated the number of surface states to obtain the statistical mechanical entropy associated with these horizons. We showed that, irrespective of the values of charges, to leading order the entropy is always proportional to the area $a_{0}$. That is, for large black holes, the number of micro-states of the horizon geometry completely determines the state-counting; there are no independent surface states from the matter sector. This explains the geometrical nature of the Bekenstein-Hawking entropy.

Perhaps the most intriguing feature of this analysis is that it rests on a delicate and subtle interplay between general relativity which provides the isolated horizon boundary conditions, quantum theory of geometry which leads to a 'polymer picture' of geometry in the bulk and quantum Chern-Simons theory which describes the geometry of the quantum-horizon. First, the ingredients needed for quantization of the Chern-Simons theory - the symplectic structure and the punctures labelled by spins - are provided, respectively, by general relativity and quantum theory of geometry. Second, the quantum-horizon boundary condition can be meaningfully imposed only because the spectra of certain quantum geometry operators on the volume Hilbert space and those of certain other operators on the Chern-Simons Hilbert space match exactly eventhough the two calculations are completely independent. This matching is in turn possible because the symplectic structure dictated by classical general relativity provides a specific value of the 
'level' $k$ of the Chern-Simons theory. Finally, the bulk quantum theory provided by quantum geometry and the surface quantum theory provided the Chern-Simons theory match seamlessly to provide a full, coherent theory. In particular, in the final picture, the quantum-horizon boundary condition - which came from the structure of isolated horizons in general relativity - can be interpreted as requiring that the bulk and the surface states be so intertwined that the total state is gauge invariant.

There are three outstanding open issues:

i) First, the classical framework developed in [1] only allowed undistorted, non-rotating black holes. However, these restrictions were removed recently [3]. Furthermore, as discussed below, there is a welldefined sense in which the quantum framework developed here already incorporates distortion. The first outstanding problem is to extend it further to incorporate rotation. Away from extremality, this is also an open issue in approaches based on string theory.

ii) The second major issue is to gain a better understanding of the Barbero-Immirzi parameter $\gamma$. While this parameter plays no physical role in the classical theory, quantum sectors corresponding to different values of $\gamma$ are unitarily inequivalent. Just as one needs an external input to resolve the $\theta$-ambiguity in $\mathrm{QCD}$, here we need experimental data (e.g., on values of the quanta of the geometric operators), or, a theoretical principle to resolve the $\gamma$-ambiguity and arrive at an unique quantum gravity theory. The requirement that the non-perturbative framework should yield the Bekenstein-Hawking entropy for one large isolated horizon suffices to select a specific value of $\gamma$. Then agreement continues to hold for all non-rotating isolated horizons, including the cosmological ones, irrespective of the values of charges and the cosmological constant. Further considerations [45] show that the framework also yields the correct Hawking temperature for the same value of $\gamma$. Thus, the 'external' requirement that the non-perturbative framework agree with quantum field theory in curved spacetimes with large isolated horizons suffices to remove the ambiguity and fix the framework. We now need to develop further semi-classical tests to check the robustness of this conclusion. Another avenue that might shed light on the preferred value of $\gamma$ is suggested by Carlip's approach to entropy [42], based on symmetries. A detailed examination of symmetries associated with the isolated horizon and their action on quantum states in various $\gamma$-sectors may provide new insights. However, in their present 
form, these symmetry ideas are not well-adapted to the non-rotating case and so their implementation may well have to await the incorporation of rotation.

iii) Finally, there is the issue of a systematic derivation of the Hawking radiation. First steps in this direction have already been taken [45]. Using properties of our surface states, one can refer to the rather general arguments given by Bekenstein [46] to conclude that the spectrum should be thermal. However, as usual [37], the intensity distribution contains a multiplicative factor involving the absorption cross-section of the horizon. A derivation of this cross-section from full quantum gravity is still missing. A systematic study of the semi-classical approximation may be necessary before one can carry out this calculation.

This completes the summary of major open issues. Since our analysis uses ideas from several distinct areas of mathematical physics, we conclude by making a number of remarks to clarify the role of various structures and the relation of this approach to some others.

1. Degrees of freedom: this approach. We began our analysis with a classical system: the sector of general relativity admitting a nonrotating isolated horizon with given area (and charges). We used the boundary conditions to eliminate all components of the $\mathrm{SU}(2)$ connection at the horizon other than the $\mathrm{U}(1)$ connection $W$, which alone appears in the surface term of the symplectic structure (7). Now, the boundary conditions (6) imply that, given the value $a_{0}$ of area, $W$ is unique up to gauge transformations and diffeomorphisms [1]. Thus, in the classical theory, there are no true ('configuration space') degrees of freedom on the horizon. Indeed, if there were, the quantum Hilbert space would have been infinite dimensional even for fixed values of horizon parameters. (Recall that if the classical configuration space is $\mathbb{R}^{n}$, the quantum Hilbert space is $L^{2}\left(\mathbb{R}^{n}\right)$.)

However, in the passage to quantum theory, we did not first 'solve' the boundary condition (6) on $W$ and $\underline{\Sigma}$ classically. Rather, we imposed the horizon boundary condition as an operator restriction (12) on allowed quantum states. This procedure was motivated by the physical expectation that the horizon 'should be allowed to fluctuate' in the quantum theory. Thus, we did not freeze either the connection $W$ nor the pullback $\underline{\Sigma}$ of the triad 2-form to the horizon. Both are allowed to fluctuate, but must do so 'in tandem', respecting (12). The quan- 
tum boundary condition (12) then led us to the Chern-Simons theory with punctures. This theory has a finite number of states because the phase space of the classical Chern-Simons theory with a given set of punctures is compact, reflecting the fact that the classical theory does not have 'true' configuration degrees of freedom. These states describe the geometry of the quantum horizon and account for entropy. Thus, thanks to the strategy of incorporating the horizon boundary condition through (12), we could fulfill the statistical mechanical expectation that a single, physical macrostate should correspond to a large number of micro-states and, furthermore, arrive at these micro-states through quantization.

2. Degrees of freedom: comparison. In string theory, as well as in the analysis based on symmetries a la Carlip [42], one first quantizes a larger system with true degrees of freedom and then associates the given black hole (with specific values of charges) with a finite dimensional subspace of the infinite dimensional Hilbert space of that larger system. Thus, rather than first restricting and then quantizing, one first quantizes and then restricts. Note that this is qualitatively analogous to our strategy with the quantum boundary conditions: We first ignored the boundary conditions and quantized a larger system and then projected to its subspace on which (12) holds. Of course, the larger systems are quite different in the three cases.

'Edge states' introduced in other contexts in the literature have the flavor of the surface states we introduced in this paper. However, there are some important differences. In [47], for example, techniques used in the quantum Hall effect were carried over to canonical gravity using 'geometrodynamics'. However, in that discussion, the boundary conditions at internal boundaries are not explicitly stated and so the discussion is not specific to horizons. Furthermore, it is assumed that the conditions should be such that only those (spatial) diffeomorphisms which are identity on the internal boundary are regarded as gauge. This strategy plays a key role in their construction of an infinite set of boundary observables. In our treatment, on the other hand, the specific horizon boundary conditions dictated what is 'gauge'. Furthermore, we were led to regard all spatial diffeomorphisms which map the horizon $S$ to itself as gauge. Indeed, without this, our entropy would have been infinite! Thus the 'origin' and physical meaning of surface states is quite different in the two cases. This point also serves as a key distinction 
between our approach and that of [48]. Indeed, in contrast to these analyses $[47,48]$, in our approach there are no independent surface degrees of freedom at the classical level. Independent surface states arise in quantum theory only because the quantum configuration space is larger than the classical, admitting distributional connections. By contrast, in obtaining the 'edge states' and 'surface degrees of freedom' in $[47,48]$ these functional analytical subtleties play no role. Finally, there is some similarity between our surface states and those considered in [41]. However, that analysis refers to Euclidean gravity and uses boundary conditions which are unrelated to the presence of horizons. There is no $\mathrm{U}(1)$ reduction on the boundary so that the Chern-Simons theory considered there refers to $\mathrm{SU}(2)$. Finally, the boundary in [41] is the outer boundary, not inner. Thus, while these three treatments share some features of our analysis, they consider quite different physical situations and their full mathematical frameworks are also different from ours.

3. Horizon boundary conditions. Note that the isolated horizon boundary conditions play several distinct roles in our classical analysis $[1,2]$. First, they enable us to show that the full $\mathrm{SU}(2)$ connection $\underline{A}$ at the horizon can be recovered from the $\mathrm{U}(1)$ connection $W$. Second, they lead us to the surface term in the symplectic structure involving $W$. This structure brings out the relation between the 'total', the 'volume' and the 'surface' phase spaces, described at the end of Section 2.1, suggesting that the space of quantum states should have an analogous structure. We were therefore led to begin the quantum analysis on the Hilbert space $\mathcal{H}=\mathcal{H}_{V} \otimes \mathcal{H}_{S}$. Finally, the residual relation (6) led to the quantum boundary condition (12) which played a key role in the present paper.

Because equation (12) played such an important role in this paper, one might be tempted to the ignore other roles of the boundary conditions. Indeed, this was done in [49] where the author noted that (6) does not suffice to capture the idea that $S$ represents a horizon and went on to replace that condition with another. However, as is clear from the detailed structure at the horizon discussed in $[1,2]$, the fact that the $\mathrm{SU}(2)$ connection $\underline{A}$ has a specific form - the first role of the boundary condition noted above- does suffice to ensure that $S$ is a 
marginally trapped surface. ${ }^{7}$

Nonetheless, since our quantum considerations depend primarily on (12), one might ask if there there exist contexts, other than isolated horizons, where our ideas are applicable. As pointed out in the first part of [49], the answer is in the affirmative: One can indeed construct mathematical models, even unrelated to general relativity, to which this analysis is applicable. This is not surprising because the ideas underlying polymer geometry are robust and applicable to a wide variety of situations. Of course, as noted in [49], the physical interpretation of surface quantum states and of the resulting entropy would depend on the specific context to which these ideas are applied.

4. Examples. Our isolated horizon boundary conditions are satisfied at the event horizons of the Reissner-Nordström family as well as cosmological horizons of de-Sitter space times. As discussed in detail in [2], one can construct a large family of examples by appropriately 'adding radiation' to the exterior regions of these spacetimes. In all these cases, our analysis of the geometry of quantum horizons goes through provided $M$ can be chosen as a partial Cauchy slice in an appropriate piece of these spacetimes (see Figures 5 and 6 in the second paper in Ref. [2]). Furthermore, since we trace over the volume degrees of freedom in our entropy calculation, the presence of radiation in the exterior region does not affect that analysis.

Finally, although we began the classical analysis in [1] with undistorted horizons, there is a precise sense in which distortion is automatically included in the final theory. In the classical theory, we did not explicitly restrict ourselves to spherical horizons. Rather our boundary conditions, together with standard differential geometric identities, imply that the scalar curvature of the horizon 2-metric is constant, i.e., the 2 -metric is spherically symmetric with respect to some 3 -dimensional $\mathrm{SO}(3)$ subgroup of $\operatorname{Diff}(S)$. Now, in quantum geometry, these identities are not available, whence an analogous conclusion can not be drawn.

\footnotetext{
${ }^{7}$ Furthermore, the proposed modification of [49] does not appear to be viable because the corresponding action (equation (10) of [49]) fails to be functionally differentiable with respect to $e$ unless $\underline{F}$ vanishes on the boundary, a condition which is violated already in the Schwarzschild family. By contrast, (6) holds on all nonrotating isolated horizons. However, we should add that the main confusion in [49] probably came about because, due to a space limitation, the boundary conditions were not spelled out in detail in the brief report [5].
} 
Indeed, there is a precise sense in which the quantum geometry of $S$ is flat everywhere except at the punctures. At the $i$ th puncture $p_{i}$, there is a quantized angle deficit of $4 \pi a_{i} / k$ for some integer $a_{i}$. From the classical theory of distorted horizons now available [3], one can interpret $a_{i}$ as giving us the 'strength' of the scalar curvature at $p_{i}$. Since the conditions which the integers $a_{i}$ are subject to allow them to vary from puncture to puncture, it is clear that the quantum geometry is allowed to be 'non-spherical'. More precisely, given any distorted, classical 2geometry on $S$, the Hilbert space $\mathcal{H}^{\text {Kin }}$ includes semi-classical states which, upon coarse-graining, approximate that 2-geometry. Thus, the classical limit of our quantum theory already includes all non-rotating distorted horizons. In view of the fact that the quantum geometry has discrete, distributional character, this result is not surprising. Indeed, none of our quantum states can have an exact spherical symmetry. More generally, as in the quantum theory of solids, because of the 'atomic' nature of geometry, quantum states can not have any continuous spatial symmetries.

5. Dynamics and related issues. The fact that we are dealing with general relativity dictated our choice of the phase space and the symplectic structure. Therefore, our analysis is not valid for higher derivative theories where the phase space is larger and the symplectic structure and the constraints take on a different form. Indeed, the first law of classical black hole mechanics suggests that the entropy should not be proportional to area in such theories [50].

In quantum general relativity, physical states must solve quantum constraints. In our calculations, the Gauss and the diffeomorphism constraints play an important role; in particular, without their imposition, the entropy would have been infinite. The Hamiltonian constraint, on the other hand, plays a minor role. This is because, in the classical theory, it leads to a well-defined Hamiltonian vector field on the phase space only if it is smeared with a lapse function which goes to zero on $S$ (and at infinity). 'Time evolution' along the horizon is generated not by constraints but by a 'true Hamiltonian' with a non-vanishing surface term at $S[1,2,3]$. Consequently, physical states have to be annihilated only by the constraint smeared with a lapse which vanishes at $S$, whence this constraint does not play a direct role in the determination of the geometry of the quantum horizon. What we need is only that, for generic, permissible lists $\left(j_{1}, \ldots, j_{n}\right)$ and $\left(m_{1}, \ldots, m_{n}\right)$, 
there exists a solution to the Hamiltonian constraint in the bulk (with lapse going to zero on $S$ ) constructed out of a spin network $n$ of whose edges intersect $S$ and carry labels $j$ and $m$. Thus, our construction does not depend on full dynamics. ${ }^{8}$ This feature seems to be rather general. Carlip's approach [42], for example, is based on symmetries rather than details of dynamics. Similarly, in string theory, one does not yet have control on full dynamics (e.g., the interaction between branes and anti-branes) and entropy calculations are possible precisely in those cases where they do not depend on these unresolved aspects of dynamics.

We began our analysis by restricting ourselves to spacetimes which admit isolated horizons as their inner boundaries. To ensure that no radiation falls across the portion of horizon under consideration, the allowed initial data on a spatial surface must satisfy some implicit conditions (in addition to the initial value constraints of Einstein's theory). Therefore, strictly speaking, quantization of the bulk theory is more subtle than in cases without internal boundaries. However, these subtleties should not affect the analysis of surface states which are sensitive only to the boundary conditions at the horizon. In the calculation of entropy, one traces over the bulk degrees and hence these subtleties get 'washed away'; in the detailed calculation of Section 6, we counted only surface states. This is also the reason why details of bulk spin networks such as their knotting in the bulk and the precise nature of intersection of edges with $S$ do not affect the quantum geometry and entropy of the horizon.

6. Entropy: physical considerations. The entropy we calculated is not an intrinsic attribute of the spacetime as a whole but depends on the division of the spacetime in to exterior and interior regions. Operationally, it is tied to the class of observers who live in the exterior region, for whom the isolated horizon is a physical boundary that separates the part of the spacetime they can access from the part they can not. (This is in sharp contrast to early work $[6,7]$ which focussed only on the interior.) This point is especially transparent in the case of cosmological horizons in de Sitter spacetime since that spacetime does not even ad-

\footnotetext{
${ }^{8}$ Note however that given the phase space, considerations motivated by spacetime covariance essentially determine gravitational terms in the Hamiltonian constraint [51]. In this sense, within our setting, the remaining freedom in dynamics comes essentially from matter couplings and the value of the cosmological constant.
} 
mit an invariantly defined division. Note however that, while there is an 'observer dependence' in this sense, our entropy does not refer to the number of interior degrees of freedom which are inaccessible to the observers under consideration. Indeed, on general grounds, it would seem unreasonable to associate entropy with interior states: since one can imagine multiple universes inside the horizon which do not communicate to the exterior region, the number of potential interior states compatible with the data accessible to the exterior observers is uncontrollably large. In our analysis, interior states were never mentioned. In particular, our 'tracing' was done with respect to the bulk states in the exterior. Our entropy refers to the micro-states of the boundary itself which are compatible with the macroscopic constraints on the area and charges of the horizon; it counts the physical micro-states which can interact with the outside world, and are not disconnected from it.

The goal of our framework is to answer the following question: Given that there is an isolated horizon, what is the entropy associated with it? In light of the conditional nature of this question, it was appropriate to begin with a suitably restricted sector of general relativity and then carry out quantization. However, as a result, our description is an effective one. In a fundamental description, one would first quantize the whole theory and then isolate states which admit 'quantum horizons' with given area and charges. However, since the notion of a horizon is deeply tied to classical geometry, at present it seems difficult to state precisely what one would mean by quantum horizons in a full quantum theory, irrespective of the approach one wants to use. Fortunately, for thermodynamic considerations involving large black holes, effective descriptions are adequate.

7. Comparison with results from string theory. Because one begins with classical general relativity and uses non-perturbative quantization methods, in the present approach one can keep track of the physical, curved geometry. In particular, as required by physical considerations, the micro-states which account for entropy can interact with the physical exterior of the black hole. In string theory, by contrast, detailed calculations $[52,53]$ are generally performed in flat space and nonrenormalization arguments and/or duality conjectures are then invoked to argue that the results so obtained refer to macroscopic black holes. Therefore, the relation to the curved space geometry and the physical meaning of the degrees of freedom which account for entropy is rather 
obscure. More generally, lack of direct contact with physical spacetime can also lead to practical difficulties while dealing with other macroscopic situations in string theory. For example, while we could easily account for the entropy normally associated with de Sitter horizons in four spacetime dimensions, this task appears to be rather difficult in string theory. ${ }^{9}$ On the other hand, in the study of genuinely quantum, Planck size black holes, this 'distance' from the curved spacetime geometry may turn out to be a blessing, as classical curved geometry will not be an appropriate tool to discuss physics in these situations. In particular, a description which is far removed from spacetime pictures may be better suited in the discussion of the last stages of Hawking evaporation and the associated issue of 'information loss'.

The calculations based on string theory have been carried out in a number of spacetime dimensions while the approach presented here is directly applicable only to four dimensions. An extension of the underlying non-perturbative framework to higher dimensions was recently proposed [55] but a systematic development of quantum geometry has not yet been undertaken. Also, our quantization procedure has an inherent $\gamma$-ambiguity which trickles down to the entropy calculation. By contrast, calculations in string theory are free of this problem. On the other hand, within string theory, detailed calculations (based on D-branes) have been carried out only for (a sub-class of) extremal or near-extremal black holes. While these black holes are especially simple to deal with mathematically, unfortunately, they are not of direct relevance to astrophysics, i.e., to the physical world we live in.

Away from extremality, there is another argument [56] in which the Schwarzschild black hole is regarded as a highly excited bosonic string. But it is more of a semi-qualitative estimate rather than a systematic, ab-initio calculation. In particular, while the estimated entropy does turn out to be proportional to the area, there is no control on the numerical coefficient and, moreover, when extended to include charged black holes, the coefficient seems to depend on the charge. More recently, the Maldacena conjecture has been used to calculate the entropy of non-extremal black holes. However, the numerical coefficient in front

\footnotetext{
${ }^{9}$ Recently, 2-dimensional de Sitter spacetimes were were discussed in the context of AdS/CFT duality (modulo a caveat on the existence of configurations of the required type in full string theory). However, in higher dimensions it is difficult to arrive at precise conclusions because of certain divergences [54].
} 
of the entropy can only be calculated in the free field approximation and turns out to be incorrect (see, e.g., [57]). This is not a discrepancy since the (super) gravity approximation can be trusted only for large couplings, but in this regime the entropy calculation on the field theory side seems practically impossible. Moreover, as is generally recognized, the boundary conditions used in the Maldacena duality are quite unphysical since the radius of the compactified dimensions is required to equal the cosmological radius even near infinity. Hence the relevance of these mathematically striking results to our physical world remains unclear. In the current approach, by contrast, ordinary, astrophysical black holes in the physical four spacetime dimensions are included from the beginning.

In spite of these contrasts, there are some striking similarities. Our polymer excitations resemble strings. Our horizon looks like a 'gravitational 2-brane'. Our polymer excitations ending on the horizon, depicted in Figure 2, closely resemble strings with end points on a membrane. As in string theory, our '2-brane' carries a natural gauge field. Furthermore, the horizon degrees of freedom arise from this gauge field. These similarities seem astonishing. A closer examination brings out some differences. In particular, being the horizon, our '2-brane' has a direct interpretation in terms of the curved spacetime geometry and our $\mathrm{U}(1)$ connection is the gravitational spin-connection on the horizon. Nonetheless, it is not impossible that, when quantum gravity is understood at a deeper level, it will reveal that the striking similarities are not accidental, i.e., that the two descriptions are in fact related.

\section{Acknowledgements}

The authors thank Maarten Bergvelt, Alejandro Corichi, Gary Horowitz, Minhyong Kim, Jerzy Lewandowski, Robert Israel, Ted Jacobson, Greg Kuperberg, Don Marolf, Carlo Rovelli, Daniel Sudarski and Alan Weinstein for useful discussions. The authors were supported in part by the NSF grants PHYS94-07194, PHY95-14240, INT97-22514 and by the Eberly research funds of Penn State. In addition, KK was supported by the Braddock fellowship of Penn State. JB thanks the Center for Gravitational Physics and Geometry for their hospitality while this paper was being written. All authors acknowledge the support from 
the Erwin Schrödinger Institute, Vienna where part of this work was carried out.

\section{Index of Notation}

For each symbol we list the section or sections where it is defined.

$a-$ list $\left\{a_{1}, \ldots, a_{n}\right\}$ of elements of $\mathbb{Z}_{k}$ labelling punctures. 4.3.2.

$\mathcal{A}$ - classical configuration space, space of $\mathrm{SU}(2)$ connections on $P$. $2.2,4.1$.

$\mathcal{A}^{\mathcal{P}}$ - generalized $\mathrm{U}(1)$ connections on $Q$, flat except at punctures in P. 4.3.1.

$\overline{\mathcal{A}}$ - quantum configuration space, space of generalized SU(2) connections on $P .2 .2,4.1$.

$\overline{\mathcal{A}}_{S}$ - space of generalized connections on the surface. 4.1.

$\overline{\mathcal{A}}_{V}$ - space of generalized connections in the volume. 4.1.

$\mathcal{A}_{\mathfrak{g}}$ - space of $\mathrm{SU}(2)$ connections on the graph $\mathfrak{g} .2 .2$.

$A(j)$ - area associated to list of spins $j .6 .1$.

$\hat{A}_{S}$ - operator corresponding to area of horizon surface. 4.2 .

$a_{0}$ - horizon area. 2.1.

$A_{a}^{i}-\mathrm{SU}(2)$ connection on the bundle $P$ over $M .2 .1$.

$\mathcal{D}$ - group of diffeomorphisms of $M$ generated by analytic vector fields tangential to $S$ and going to zero at infinity. 5.2.2.

$\mathcal{D}^{\mathcal{P}}$ - group of real-analytic diffeomorphisms of $S$ respecting certain structure associated with punctures in $\mathcal{P}$. 4.3.1.

$E_{i}^{a}$ - orthonormal triad of density weight one on $M .2 .1$.

$F_{a b}$ - curvature of the connection W. 3. 
$g_{i}$ - holonomy of $W$ along $\gamma_{i}$. 4.3.1.

$\mathcal{G}$ - group of not necessarily continuous gauge transformations of $P$ that equal the identity on $S .4 .2$.

$\mathcal{G}^{\mathcal{P}}$ - group of not necessarily continuous $\mathrm{U}(1)$ gauge transformations of $Q$ that equal identity at punctures in $\mathcal{P}$. 4.3.1.

$\mathcal{G}_{Q}$ - group of not necessarily continuous gauge transformations of the bundle $Q$. 5.1.1.

$\hat{\mathcal{G}}_{Q}$ - group of not necessarily continuous gauge transformations of the bundle $Q$ taking values in $\mathbb{Z}_{k}$. 5.1.1.

$\mathcal{G}_{S}$ - group of not necessarily continuous gauge transformations of the bundle $P$ restricted to $S$. 4.2.

$\mathfrak{g}$ - graph embedded in $M .2 .2,4.1$.

$h_{i}$ - holonomy of $W$ along $\eta_{i}$. 4.3.1.

$\hat{h}_{i}$ - quantum operator corresponding to $h_{i}$. 5.1.2.

$\mathcal{H}_{\mathfrak{g}}-L^{2}\left(\overline{\mathcal{A}}_{\mathfrak{g}}\right) \cdot 2.2$.

$\mathcal{H}^{\text {Kin }}$ - kinematical Hilbert space. 5.1.3.

$\mathcal{H}^{\text {Phys }}$ - physical Hilbert space. 5.2.3.

$\mathcal{H}^{\text {bh }}-$ Hilbert space of states satisfying area constraint. 6.1.

$\mathcal{H}_{S}^{\mathrm{bh}}-$ Hilbert space of surface states satisfying area constraint. 6.1.

$\mathcal{H}_{S}$ - surface Hilbert space. 4.3.2.

$\mathcal{H}_{S}^{\text {Phys }}$ - physical surface Hilbert space. 5.2.3.

$\mathcal{H}_{S}^{\mathcal{P}}$ - subspace of surface Hilbert space associated to set of punctures P. 4.3.2.

$\mathcal{H}_{S}^{\mathcal{P}, a}$ - subspace of surface Hilbert space associated to set of punctures $\mathcal{P}$ labelled by $\mathbb{Z}_{k}$ elements $a$. 5.1.2.

$\mathcal{H}_{S}^{\mathcal{S}(\mathcal{P}), a}$ - subspace of surface Hilbert space associated to set of punctures $\mathcal{P}$ labelled by $\mathbb{Z}_{k}$ elements $a$ and extra structure $\mathcal{S}$. 5.2.2. 
$\mathcal{H}_{S}^{n, a}-$ Hilbert space of surface states associated to $n$ punctures labelled by $\mathbb{Z}_{k}$ elements $a$. 5.2.2.

$\mathcal{H}_{V}$ - volume Hilbert space. 4.2 .

$\mathcal{H}_{V}^{\text {Phys }}$ - physical volume Hilbert space. 5.2.3.

$\mathcal{H}_{V}^{\mathcal{P}}$ - subspace of volume Hilbert space associated to set of punctures P. 3.2.

$\mathcal{H}_{V}^{\mathcal{P}, j}$ - subspace of volume Hilbert space associated to set of punctures $\mathcal{P}$ labelled by spins $j$. 4.2.

$\mathcal{H}_{V}^{\mathcal{P}, m}$ - subspace of volume Hilbert space associated to set of punctures $\mathcal{P}$ labelled by nonzero half-integers $m$. 4.2.

$\mathcal{H}_{V}^{n, m}$ - Hilbert space of volume states associated to $n$ punctures labelled by nonzero half-integers $m$. 5.2.3.

$\mathcal{H}_{V}^{n, m}$ - space of volume states satisfying Hamiltonian constraint associated to $n$ punctures labelled by nonzero half-integers $m$. 5.2.3.

$j-$ list of spins $\left\{j_{1}, \ldots, j_{n}\right\}$ labelling punctures. 4.2.

$J^{i}(p)$ - generator of $\mathrm{SU}(2)$ gauge rotations at point $p .2 .2,4.2$.

$K_{a}^{i}-\operatorname{Ad} P$-valued 1-form constructed from the extrinsic curvature of M. 2.1.

$k$ - 'level' in U(1) Chern-Simons theory, equal to $a_{0} / 4 \pi \gamma \ell_{P}^{2} .3$.

$k_{i j}$ - Cartan-Killing form on $\mathfrak{s u}(2)$. 2.1.

$L-$ complex line bundle over $\mathcal{X}^{\mathcal{P}}$. 4.3.2.

$\Lambda$ - the lattice $(2 \pi \mathbb{Z})^{2(n-1)}$. 4.3.1.

$m$ - list of nonzero half-integers $\left\{m_{1}, \ldots, m_{n}\right\}$ labelling punctures. 5.1.1.

$M$ - the spatial 3-manifold, the complement of the open unit ball in $\mathbb{R}^{3}$. 2.1.

$N_{\mathrm{bh}}$ - number of black hole surface states. 6.1. 
$P$ - trivial SU(2) bundle over S. 2.1.

$\mathcal{P}$ - finite set $\left\{p_{1}, \ldots, p_{n}\right\}$ consisting of 'punctures' $p_{i}$, namely points in $S .3 .2,4.3 .1$.

$q_{a b}$ - the 3-metric, Riemannian metric on M. 2.1.

$Q$ - spin bundle of horizon surface, a U(1) sub-bundle of $P$ restricted to $S .2 .1$.

$r$ - unit internal vector field on horizon surface, a smooth function from $S$ to $\mathfrak{s u}(2) .2 .1$.

$R$ - projective representation of translation group on holomorphic functions on $\mathbb{C}^{n-1}$, giving representation of $\mathbb{Z}_{k}^{n}$ on $\mathcal{H}_{S}^{\mathcal{P}}$. 4.3.2, 5.1.2.

$S$ - the horizon surface, unit sphere in $\mathbb{R}^{3}$. 2.1.

$\mathcal{S}$ - extra structure on horizon surface. 4.3.1.

$S_{\mathrm{bh}}$ - black hole entropy. 6.1 .

$W-\mathrm{U}(1)$ connection on $Q$ constructed from the Levi-Civita connection on $S .2 .1$.

$x_{i}$ - coordinates on $\mathcal{X}^{\mathcal{P}}$. 4.3.1.

$X_{i}-1$-forms on $S-\mathcal{P}$. 4.3.1.

$\mathcal{X}$ - kinematical phase space. 2.1.

$\tilde{\mathcal{X}}$ - physical phase space. 2.1 .

$\mathcal{X}^{\mathcal{P}}$ - surface phase space associated with set of punctures $\mathcal{P}$. 4.3.1.

$\mathcal{X}_{S}$ - surface phase space. 2.1.

$\mathcal{X}_{V}$ - volume phase space. 2.1 .

$Y_{i}-1$-forms on $S-\mathcal{P}$. 4.3.1.

$y_{i}$ - coordinates on $\mathcal{X}^{\mathcal{P}}$. 4.3.1.

$\gamma$ - the Barbero-Immirzi parameter. 2.1. 
$\gamma_{0}$ - special value of Barbero-Immirzi parameter, equal to $\ln 2 / \pi \sqrt{3}$.

6.1, 6.2.

$\gamma_{i}$ - paths in $S .4 .3 .1$.

$\Gamma_{a}^{i}-\mathrm{SU}(2)$ connection on $P$ constructed from the Levi-Civita connection on $M .2 .1$.

$\eta_{i}-$ loops in $S .4 .3 .1$.

$\mu$ - uniform measure on $\overline{\mathcal{A}}$. $2.2,4.1$.

$\mu_{\mathfrak{g}}$ - Haar measure on $\mathcal{A}_{\mathfrak{g}} \cdot 2.2$.

$\Phi-$ diffeomorphism from $\mathcal{X}^{\mathcal{P}}$ to torus. 4.3.1.

$\psi_{a}^{\mathcal{P}}$ - basis of $\mathcal{H}_{S}^{\mathcal{P}} \cdot 4.3 .2$

$\rho_{\text {bh }}$ - black hole density matrix. 6.1.

$\Sigma_{a b}^{i}-\operatorname{Ad} P$-valued 2-form on $M$, canonically conjugate to $A$. 2.1.

$\Sigma_{a b}^{i}-$ pullback of $\Sigma_{a b}^{i}$ to horizon surface $S .2 .1$.

$\underline{\Sigma}_{a b}^{i}-$ quantum operator corresponding to $\underline{\Sigma}_{a b}^{i}$. 3 .

$\omega$ - symplectic structure on $\mathcal{X}^{\mathcal{P}}$. 4.3.1.

$\Omega_{\text {grav }}$ - symplectic structure on $\mathcal{X} .2 .1$.

\section{References}

[1] A. Ashtekar, A. Corichi, and K. Krasnov, Isolated horizons: the classical phase space, Adv. Theo. Math. Phys., 3, 418-471.

[2] A. Ashtekar, C. Beetle, and S. Fairhurst, Isolated horizons: a generalization of black hole mechanics, Class. Quantum Grav., 16 (1999), L1-L7;

A. Ashtekar, C. Beetle, and S. Fairhurst, Mechanics of isolated horizons, Class. Quantum Grav., 17 (2000), 253-298. 
[3] A. Ashtekar and A. Corichi, Laws governing isolated horizons: inclusion of dilaton couplings, Class. Quantum Grav., 17, (2000) 1317-1332;

A. Ashtekar, S. Fairhurst and B. Krishnan, Isolated horizons: Hamiltonian evolution and the first law, Phys. Rev., D (in press); A. Ashtekar, C. Beetle, O. Dreyer, S. Fairhurst, B. Krishnan, J. Lewandowski, and J. Wisniewski, Generic isolated horizons and their applications, Phys. Rev. Lett. (in press);

C. Beetle, Isolated horizons and black hole mechanics, Penn State Ph.D. Dissertation.

[4] G. Gibbons and S.W. Hawking, Cosmological event horizons, thermodynamics, and particle creation, Phys. Rev., D15 (1977), 27382751.

[5] A. Ashtekar, J.C. Baez, A. Corichi, K. Krasnov, Quantum geometry and black hole entropy, Phys. Rev. Lett., 80 (1998), 904-907.

[6] K. Krasnov, Geometrical entropy from loop quantum gravity, Phys. Rev., D55 (1997), 3505-3513;

K. Krasnov, On statistical mechanics of Schwarzschild black holes, Gen. Rel. Grav., 30 (1998), 53-68.

[7] M. Barreira, M. Carfora, and C. Rovelli, Physics with nonperturbative quantum gravity: radiation from a quantum black hole, Gen. Rel. Grav., 28 (1996), 1293-1299;

C. Rovelli, Black hole entropy from loop quantum gravity, Phys. Rev. Lett., 14 (1996), 3288-3291;

C. Rovelli, Loop quantum gravity and black hole physics, Helv. Phys. Acta., 69 (1996), 582-611.

[8] A. Ashtekar and C.J. Isham, Representation of the holonomy algebras of gravity and non-Abelian gauge theories, Class. Quant. Grav., 9 (1992), 1433-1467.

[9] A. Ashtekar and J. Lewandowski, Representation theory of analytic holonomy algebras, in 'Knots and Quantum Gravity', edited by J.C. Baez, Oxford U. Press, Oxford, 1994.

[10] J.C. Baez, Generalized measures in gauge theory, Lett. Math. Phys., 31 (1994), 213-223. 
[11] A. Ashtekar and J. Lewandowski, Projective techniques and functional integration, Jour. Math. Phys., 36 (1995), 2170-2191.

[12] A. Ashtekar and J. Lewandowski, Differential geometry on the space of connections using projective techniques, Jour. Geo. \& Phys. 17, 191-230 (1995).

[13] D. Marolf and J. Mourão, On the support of the AshtekarLewandowski measure, Commun. Math. Phys., 170 (1995), 583606.

[14] R. Penrose, Angular momentum: an approach to combinatorial space-time, in 'Quantum Theory and Beyond', edited by Ted Bastin, Cambridge University Press, 1971.

[15] C. Rovelli and L. Smolin, Spin networks and quantum gravity, Phys. Rev., D52 (1995), 5743-5759.

[16] J.C. Baez, Spin nètworks in non-perturbative quantum gravity, in 'The Interface of Knots and Physics', edited by L. Kauffman, American Mathematical Society, Providence, 1996, pp. 167-203.

[17] J.C. Baez, Spin networks in gauge theory, Adv. Math., 117 (1996), 253-272.

[18] A. Ashtekar, J. Lewandowski, D. Marolf, J. Mourão, and T. Thiemann, Quantization of diffeomorphism invariant theories of connections with local degrees of freedom, Jour. Math. Phys., 36 (1995), 6456-6493.

[19] C. Rovelli and L. Smolin, Discreteness of area and volume in quantum gravity, Nucl. Phys., B442 (1995), 593-622; Erratum: Nucl. Phys., B456 (1995), 753.

[20] A. Ashtekar and J. Lewandowski, Quantum theory of geometry I: Area operators, Class. Quant. Grav., 14 (1997), A55-A81.

[21] A. Ashtekar and J. Lewandowski, Quantum theory of geometry II: Volume Operators, Adv. Theo. Math. Phys., 1 (1997), 388-429.

[22] T. Thiemann, A length operator for canonical quantum gravity, Jour. Math. Phys., 39 (1998), 3372-3392. 
[23] J.C. Baez and S. Sawin, Functional integration on spaces of connections, Jour. Funct. Analysis, 150 (1997), 1-27;

J.C. Baez and S. Sawin, Diffeomorphism-invariant spin network states, Jour. Funct. Analysis, 158 (1998), 253-266.

[24] J. Lewandowski and T. Thiemann, Diffeomorphism invariant quantum field theories of connections in terms of webs, Class. Quant. Grav., 16 (1999), 2299-2322.

[25] A. Ashtekar, Lectures on non-perturbative canonical gravity, Notes prepared in collaboration with R.S. Tate, World Scientific, Singapore, 1991.

[26] F. Barbero, Real Ashtekar variables for Lorentzian signature spacetimes, Phys. Rev., D51 (1996), 5507-5510;

G. Immirzi, Quantum gravity and Regge calculus, Nucl. Phys. Proc. Suppl., 57 (1997), 65-72;

C. Rovelli and T. Thiemann, The Immirzi parameter in quantum general relativity, Phys. Rev., D57 (1998), 1009-1014;

R. Gambini, O. Obregon, and J. Pullin, Yang-Mills analogues of the Immirzi ambiguity, Phys. Rev., D59 (1999), 047505.

[27] T. Thiemann, Anomaly-free formulation of nonperturbative, four-dimensional Lorentzian quantum gravity, Phys. Lett., B380 (1996), 257-264;

T. Thiemann, Quantum spin dynamics, Class. Quant. Grav., 15 (1998), 839-873.

[28] C. Di Bartolo, R. Gambini, J. Griego, and J. Pullin, Consistent canonical quantization of general relativity in the space of Vassiliev invariants, gr-qc/9909063.

[29] V. Bargmann, On a Hilbert space of analytic functions and an associated integral transform, Part I, Comm. Pure Appl. Math., 14 (1961), 187-214.

[30] I.E. Segal, The complex-wave representation of the free boson field, in 'Topics in Functional Analysis', edited by I. Gohberg and M. Kac, Adv. Math. Suppl. Studies, Vol. 3, Academic Press, New York, (1978), 321-343.

[31] D. Mumford, Tata Lectures on Theta I, Birkhäuser, Boston, 1983. 
[32] P. Cartier, Quantum mechanical commutation relations and theta functions, Proc. Symp. Pure Math., 9 (1966), 361-386.

[33] M.F. Atiyah, The Geometry and Physics of Knots, Cambridge U. Press, Cambridge, 1990.

[34] S. Majid, Foundations of Quantum Group Theory, Cambridge U. Press, Cambridge, 1995.

[35] D. Mumford, M. Nori, and P. Norman, Tata Lectures on Theta III, Birkhäuser, Boston, 1991.

[36] M. Hirsch, Differential Topology, Springer, New York, 1976.

[37] S.W. Hawking, Particle creation by black holes, Commun. Math. Phys., 43 (1975), 199-220.

[38] A. Ashtekar and M. Pierri, Probing quantum gravity through exactly soluble minisuperspaces, J. Math. Phys., 37 (1996), 62506270 ;

A. Ashtekar, Large quantum gravity effects: Unforeseen limitations of classical theory, Phys. Rev. Lett., 77 (1996), 4864-4867;

M. Varadarajan, On the metric operator for quantum cylindrical waves, Class. Quant. Grav., 17 (2000), 189-199.

[39] J. Wheeler, It from bit, in 'Sakharov Memorial Lectures on Physics, Volume 2', edited by L. Keldysh and V. Feinberg, Nova Science, New York, 1992.

[40] R.K. Kaul and P. Majumdar, Quantum black hole entropy, Phys. Lett., B439 (1998), 267-270.

[41] L. Smolin, Linking topological quantum field theory and nonperturbative quantum gravity, J. Math. Phys., 36 (1995), 6417-6455.

[42] S. Carlip, Black hole entropy from conformal field theory in any dimension, Phys. Rev. Lett., 82 (1999), 2828-2831.

[43] R.K. Kaul and P. Majumdar, Logarithmic corrections to the Bekenstein-Hawking entropy, Phys. Rev. Lett., 84 (2000), 52555257.

[44] S. Carlip, Logarithmic corrections to black hole entropy from the Cardy formula, gr-qc/0005017. 
[45] K. Krasnov, Quantum geometry and thermal radiation from black holes, Class. Quant. Grav., 16 (1999), 563-578.

[46] J.D. Bekenstein, Black holes and entropy, Phys. Rev. D7 (1973), 2333-2346;

J.D. Bekenstein, Generalized second law of thermodynamics in black hole physics, Phys. Rev., D9 (1974), 3292-3300;

J.D. Bekenstein and A. Meisels, Einstein $A$ and $B$ coefficients for a black hole, Phys. Rev., D15 (1977), 2775-2781.

[47] A.P. Balachandran, L. Chandar, and A. Momen, Edge states in gravity and black hole physics, Nucl. Phys., B461 (1996), 581-596; A.P. Balachandran, L. Chandar, and A. Momen, Edge states and entanglement entropy, Int. Jour. Mod. Phys., A12 (1997), 625-642.

[48] A. Momen, Edge dynamics for BF theories and gravity, Phys. Lett., 394 (1997), 269-274.

[49] V. Husain, Apparent horizons, black hole entropy and loop quantum gravity, Phys. Rev., D59 (1999), 084919.

[50] R.M. Wald, Black hole entropy is Noether charge, Phys. Rev., D48 (1994), 3427-3431.

[51] S.A. Hojman, K. Kuchař, and C. Teitelboim, Geometrodynamics regained, Ann. Phys., 96 (1976), 88-135.

[52] A. Strominger and C. Vafa, Microscopic origin of the BekensteinHawking entropy, Phys. Lett., B379 (1996), 99-104.

[53] J. Maldacena and A. Strominger, Statistical entropy of fourdimensional extremal black holes, Phys. Rev. Lett., 77 (1996), 428429.

[54] S. Hawking, J. Maldacena, and A. Strominger, DeSitter entropy, quantum entanglement and AdS/CFT, hep-th/0002145.

[55] L. Freidel, K. Krasnov, R. Puzio, BF descriptions of higher dimensional gravity theories, Adv. Theor. Math. Phys. (in press), preprint available as hep-th/9901069. 
[56] G.T. Horowitz and J. Polchinski, A correspondence principle for black holes and strings, Phys. Rev., D55 (1997), 6189-6197; G.T. Horowitz, in Black Holes and Relativistic Stars, edited by R.M. Wald, University of Chicago Press, Chicago, 1998;

T. Damour and G. Veneziano, Self-gravitating fundamental strings and black holes, Nucl. Phys., B568 (2000), 93-119.

[57] O. Aharony, S. Gubser, J. Maldacena, H. Ooguri, and Y. Oz, Large $N$ theories, string theory and gravity, Phys. Rep., 323 (2000), 183386 . 